\title{
Hetero Diels-Alder reaction of vinyl-allenes and aldehydes. An experimental and computational study
}

David Regás, Juan M. Ruiz, María M. Afonso, J. Antonio Palenzuela*

Instituto Universitario de Bio-Orgánica “Antonio González”, Departamento de Química

Orgánica, Universidad de La Laguna, 38206 La Laguna, Tenerife, Spain

\author{
jpalenz@ull.es
}

General experimental procedures S2

Copies of ${ }^{1} \mathrm{H}$ and ${ }^{13} \mathrm{C}$ NMR spectra* of new compounds........................... S3

Discussion of the Mulliken charges of the Transition States computed ...S68 Cartesian Coordinates, energies, ZPE corrections and imaginary

frequencies for transition states for all computed compounds .S69

* The figures on the NMR spectra depicts an arbitrary enantiomer. All compounds were obtained as racemic mixtures. 
General Procedures. ${ }^{1} \mathrm{H}$ spectra were recorded at $400 \mathrm{MHz}$, and the ${ }^{13} \mathrm{C}$ NMR at 75 or 100 $\mathrm{MHz}$, in $\mathrm{CDCl}_{3}$ or $\mathrm{C}_{6} \mathrm{D}_{6}$ as solvent, and chemical shifts are reported relative to the residual solvent peak. Flash chromatography was performed on silicagel 60 (240-400 mesh) using mixtures of hexanes-ethyl acetate as eluent. Reactions requiring anhydrous conditions were carried out under and atmosphere of dry argon. Anhydrous THF was distilled from sodium in a recycling still. Other solvents were purified by standard techniques. HPLC was performed on silicagel columns $(10 \mu \mathrm{m}, \mu$-porasil) $7.8 \times 300 \mathrm{~mm}$ using mixtures of hexanes-ethyl acetate as eluent with a flowrate of $29 \mathrm{~mL} / \mathrm{min}$, using a refraction index detector. 


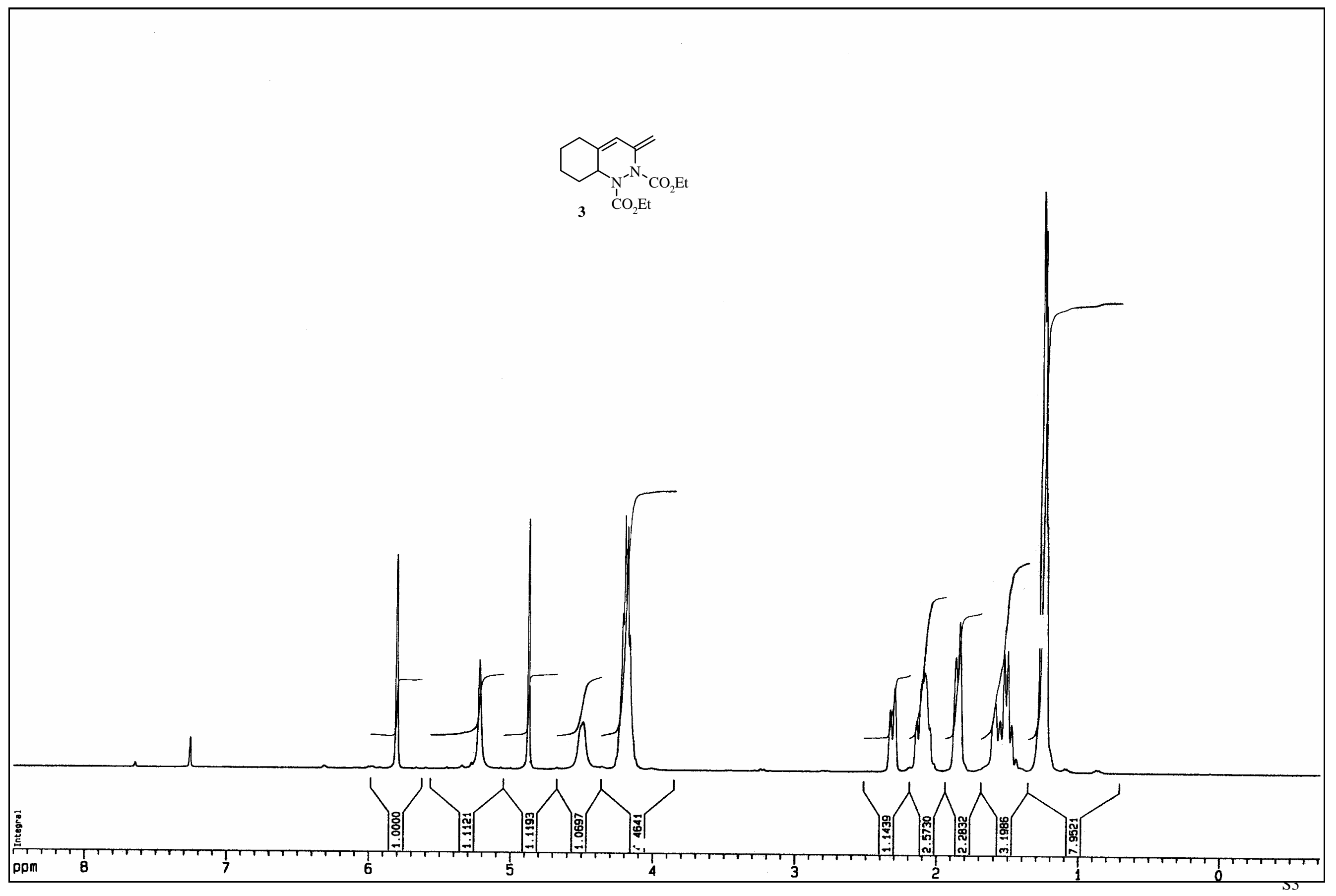




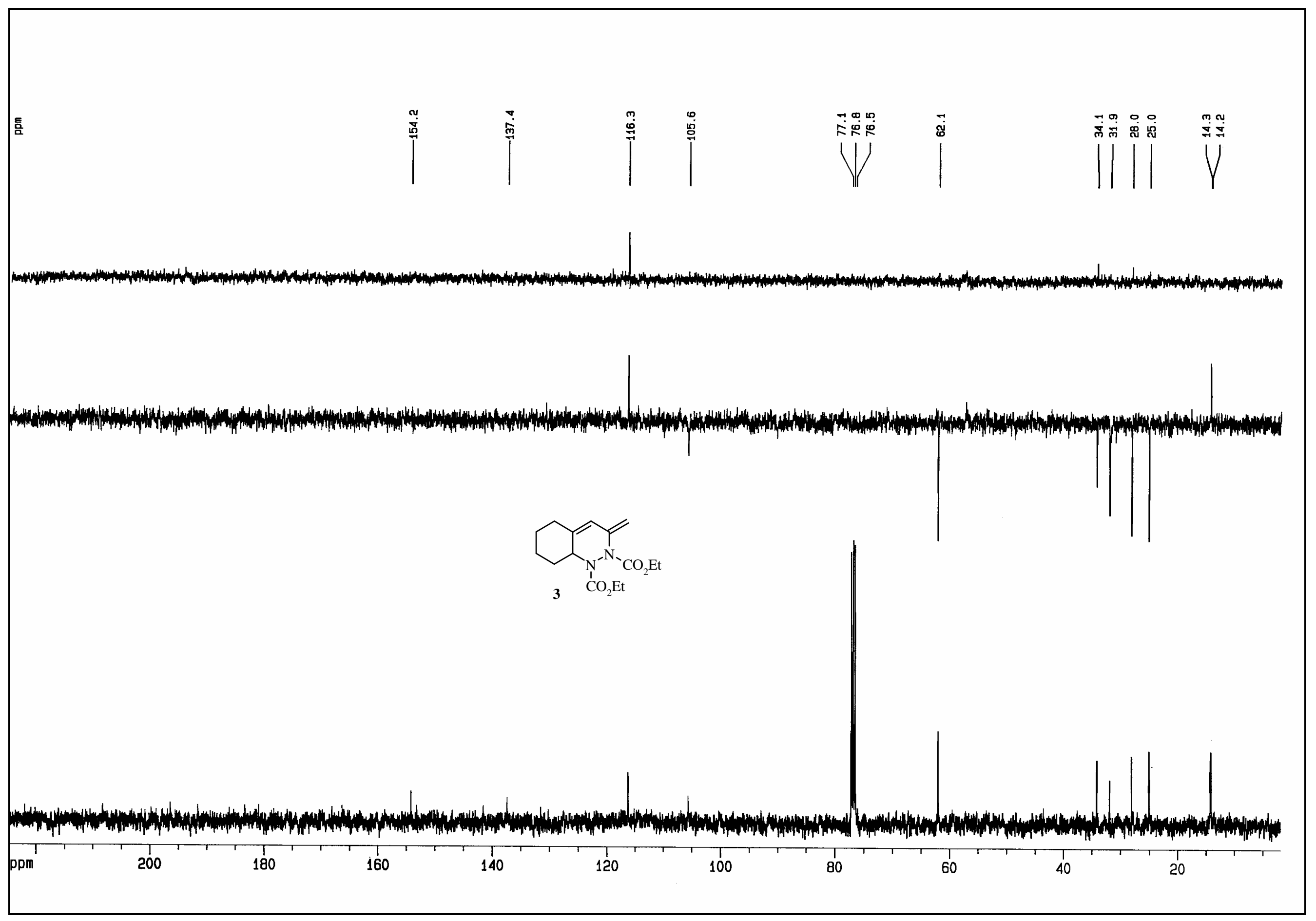




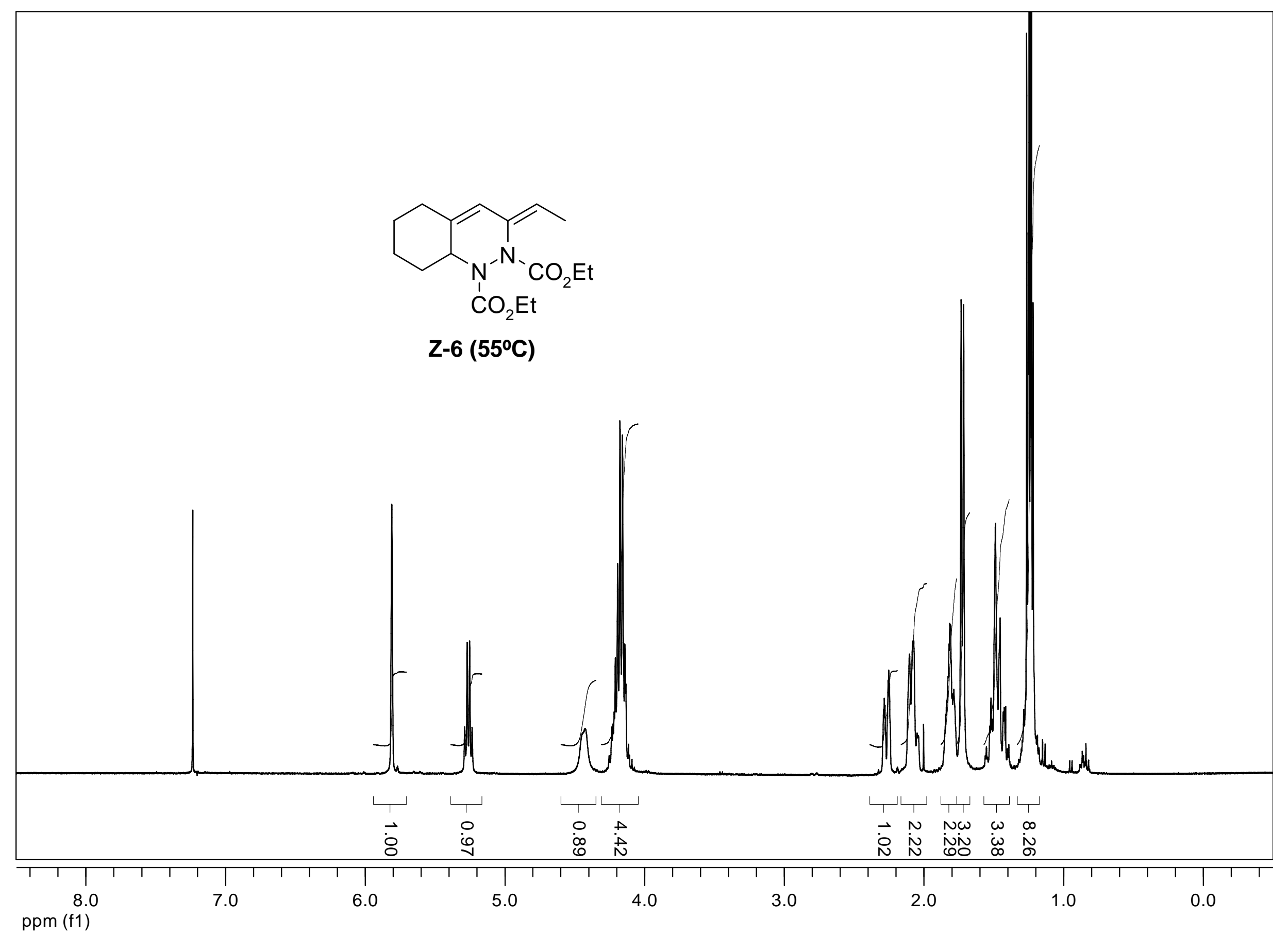




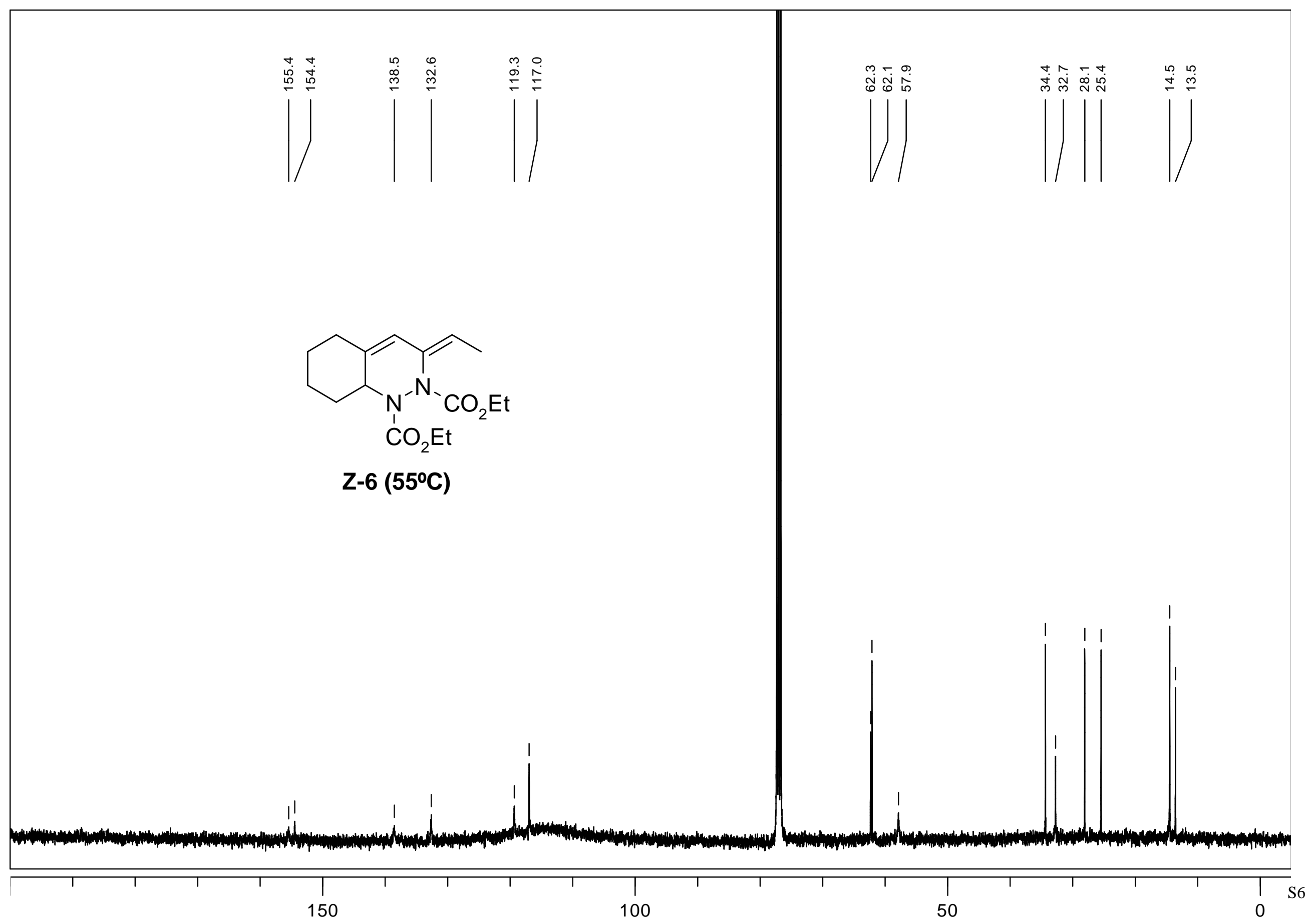

ppm (f1) 


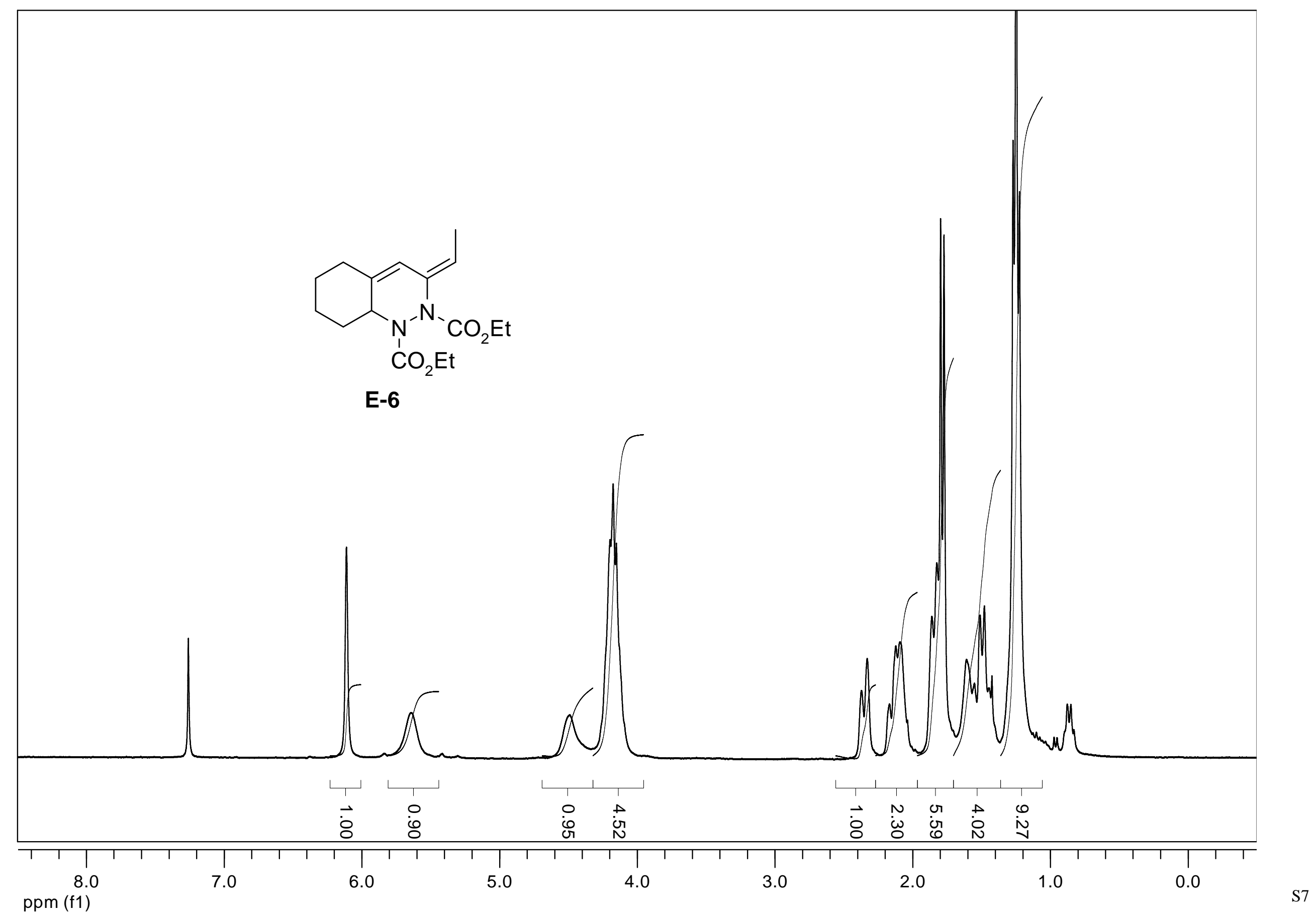




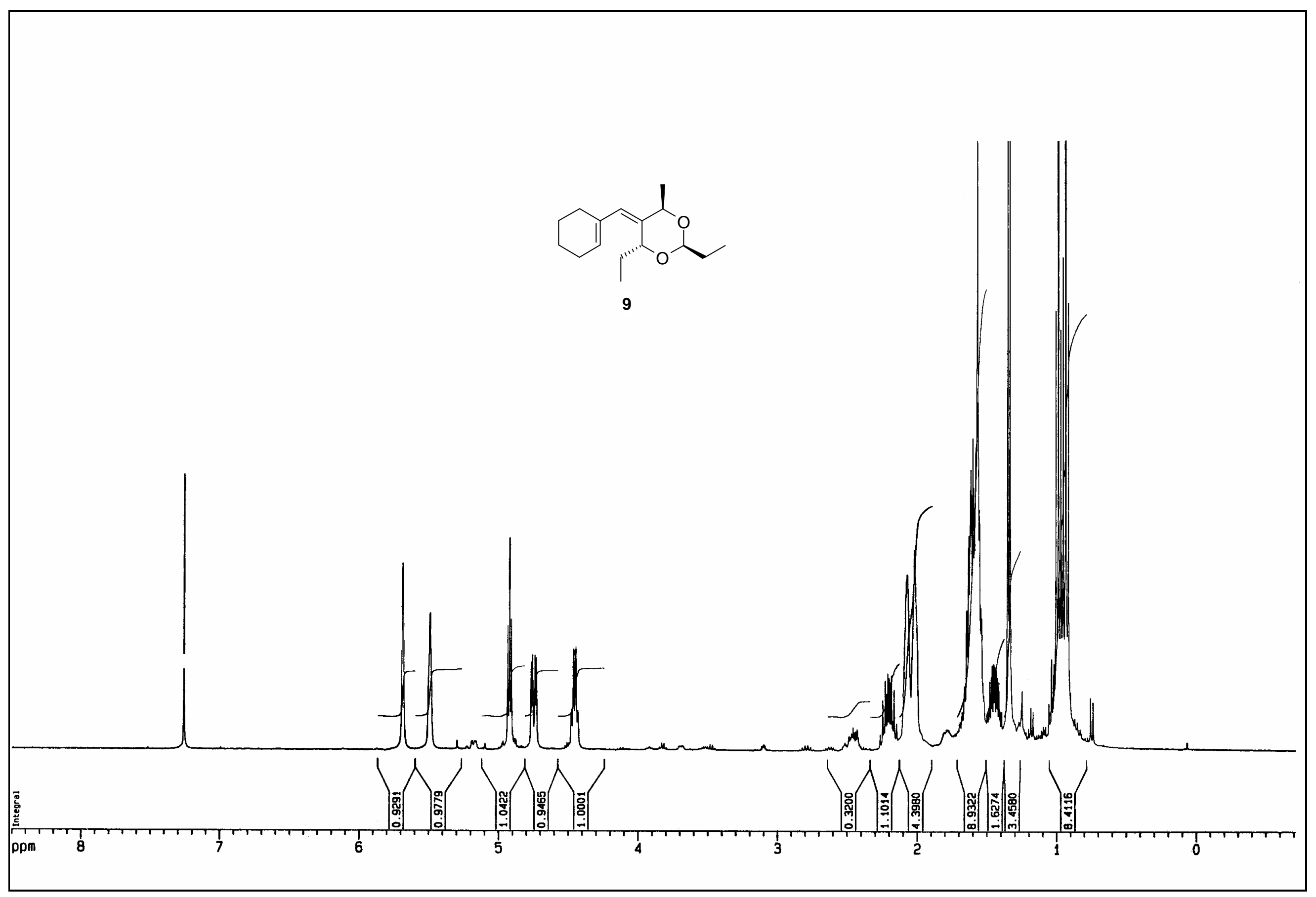




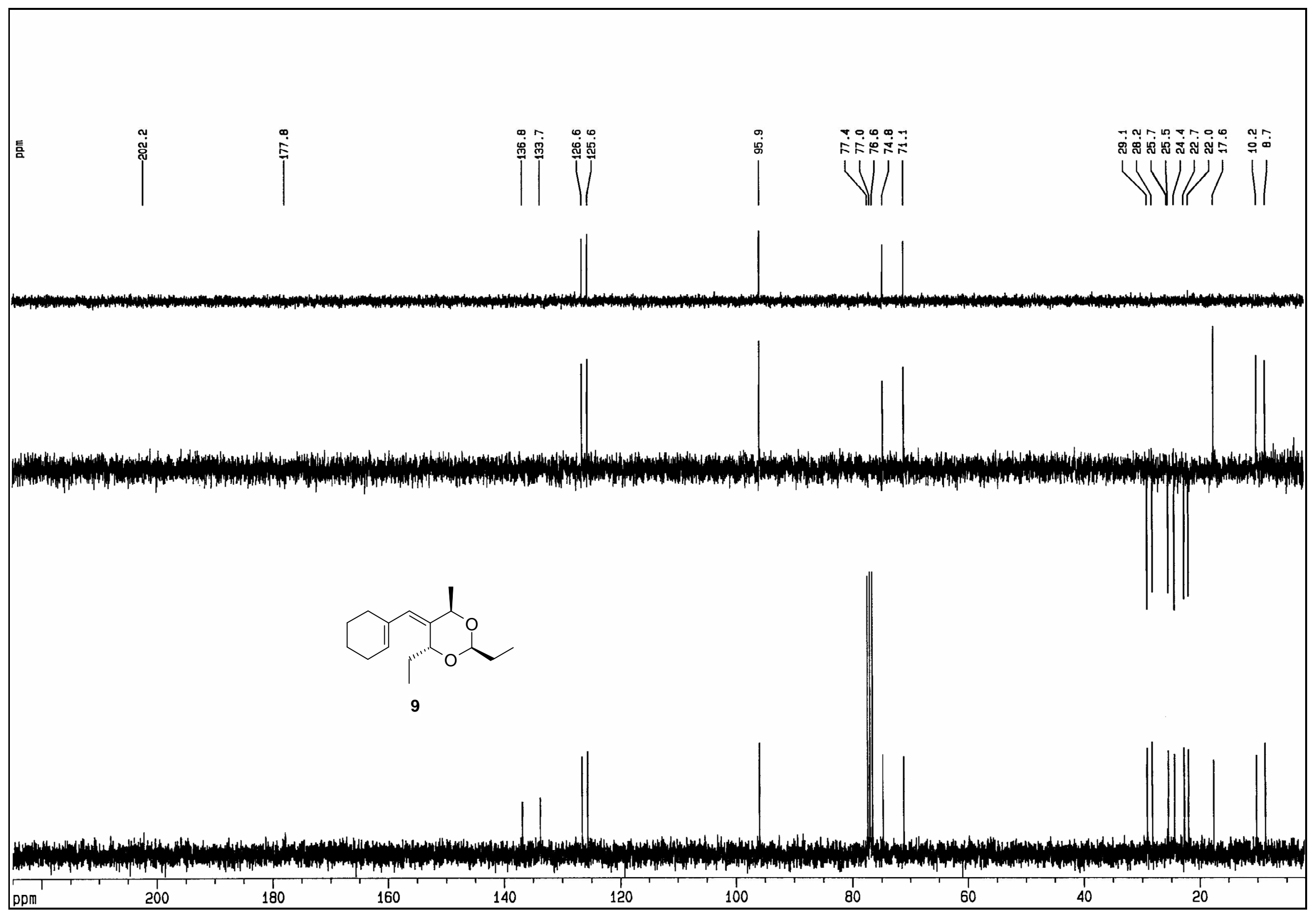




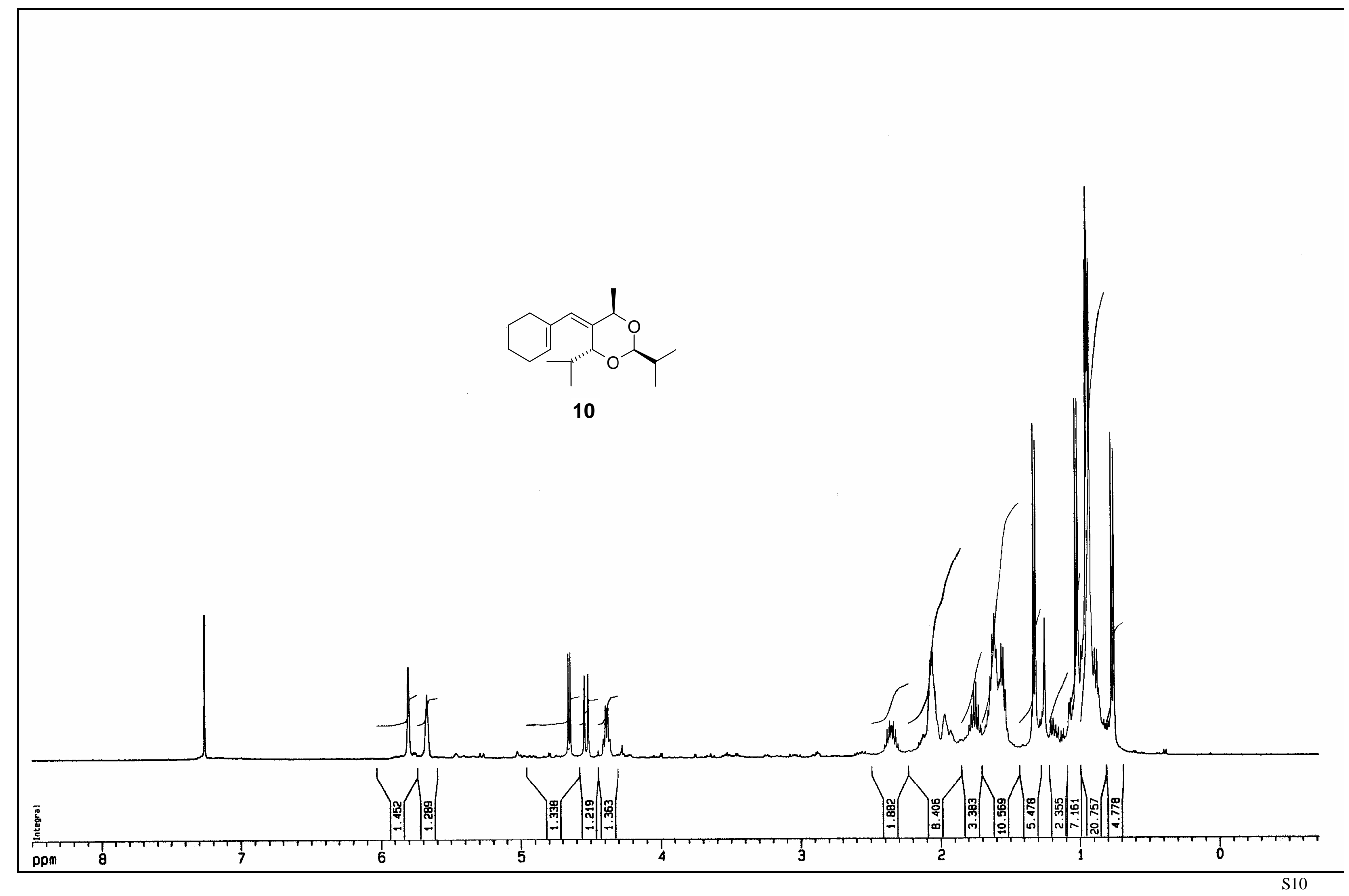




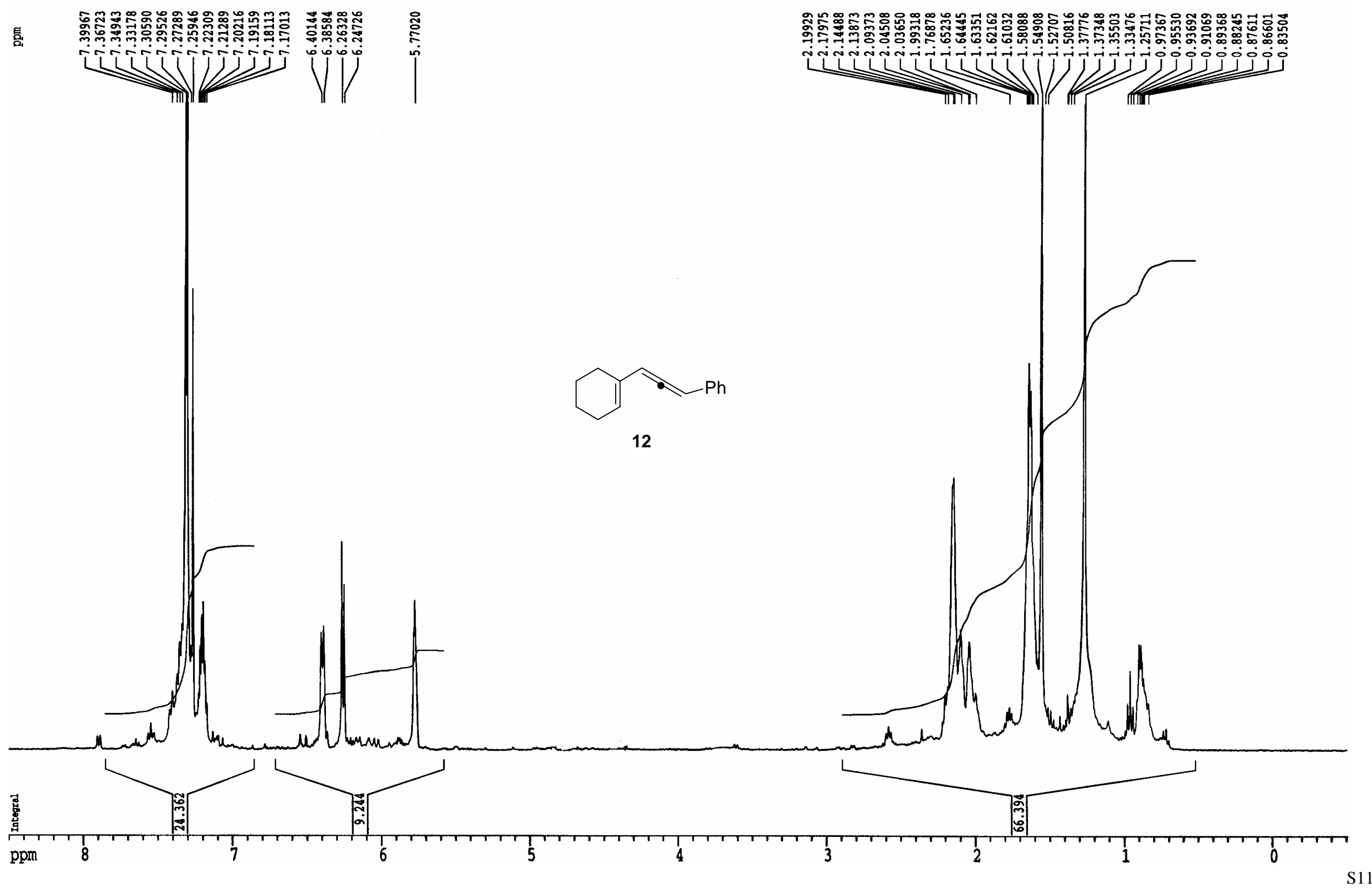




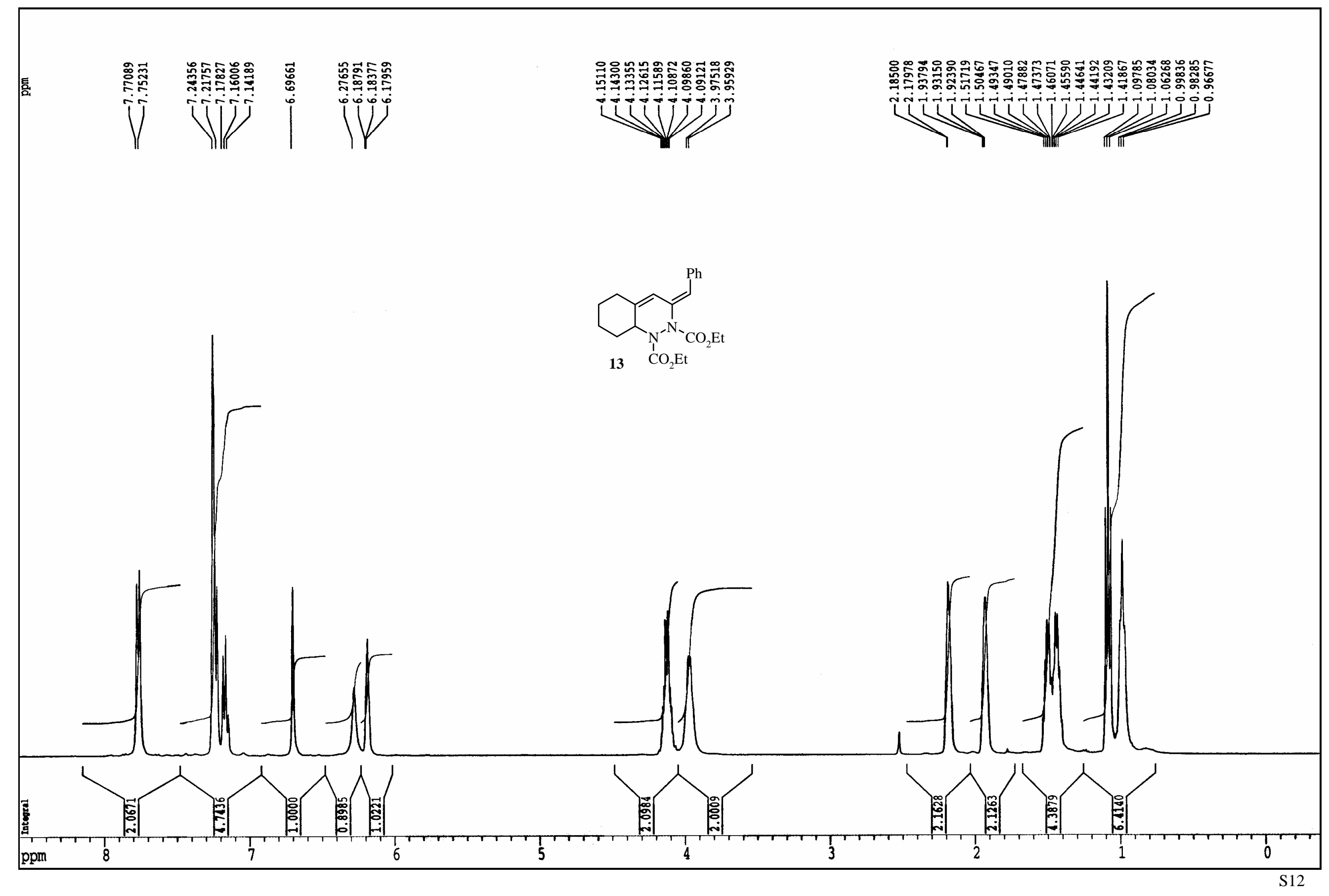




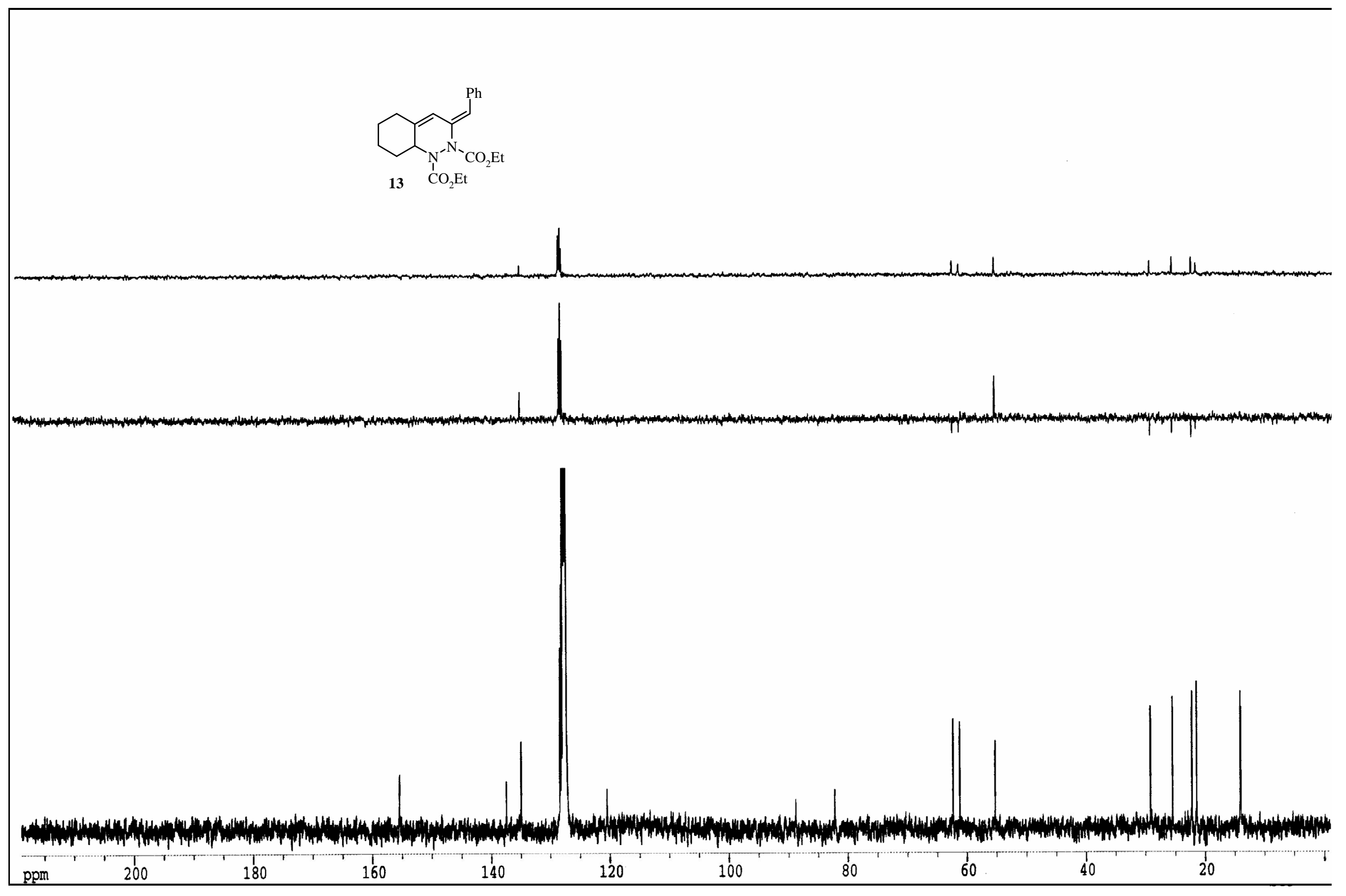




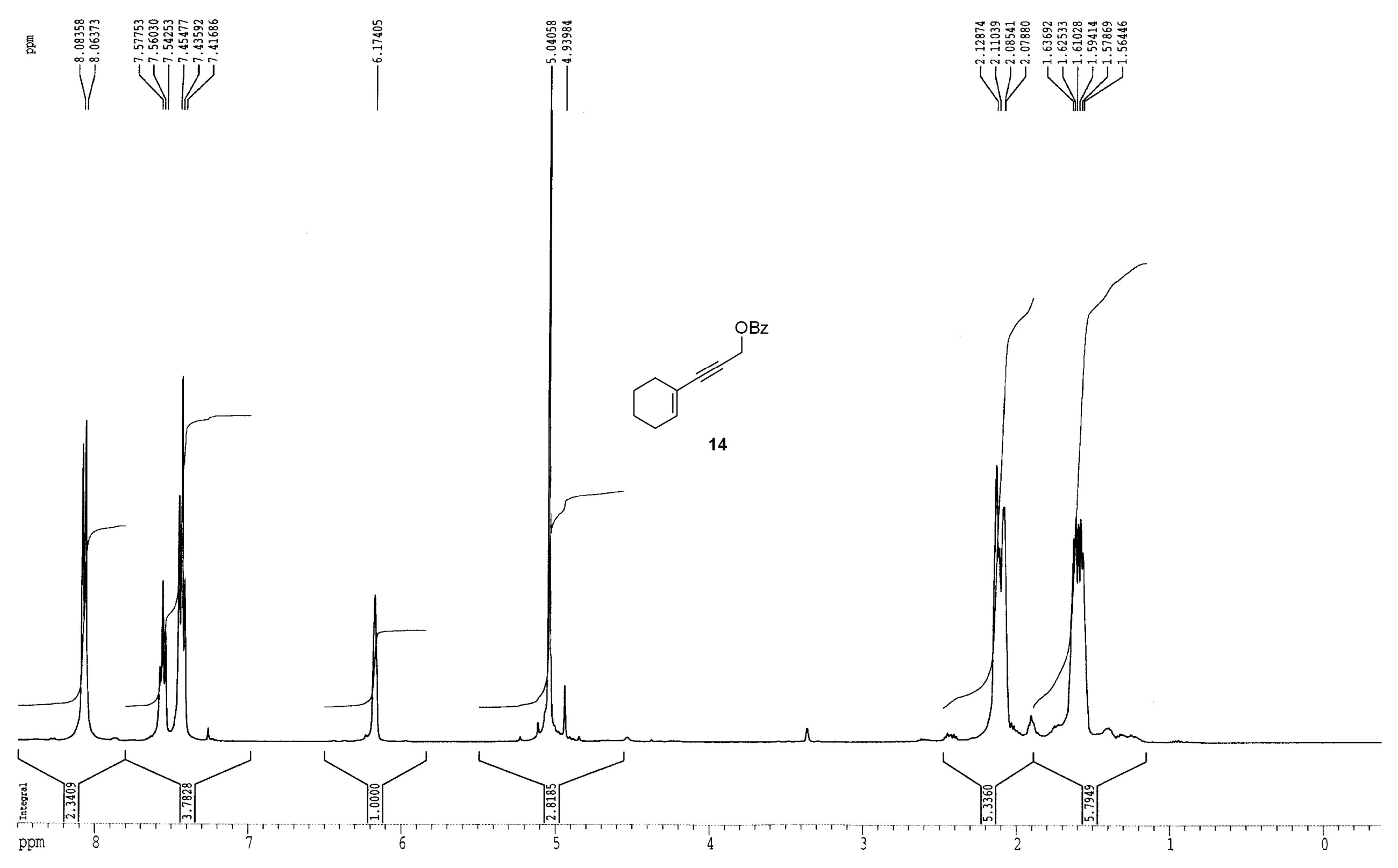




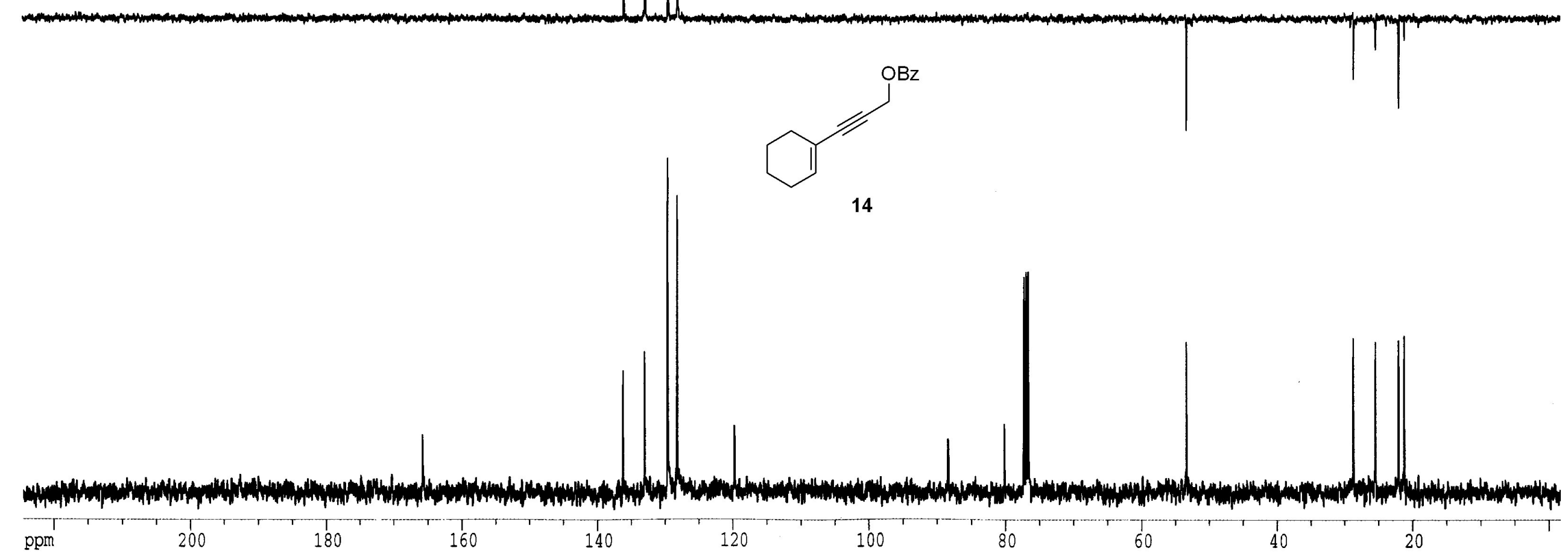




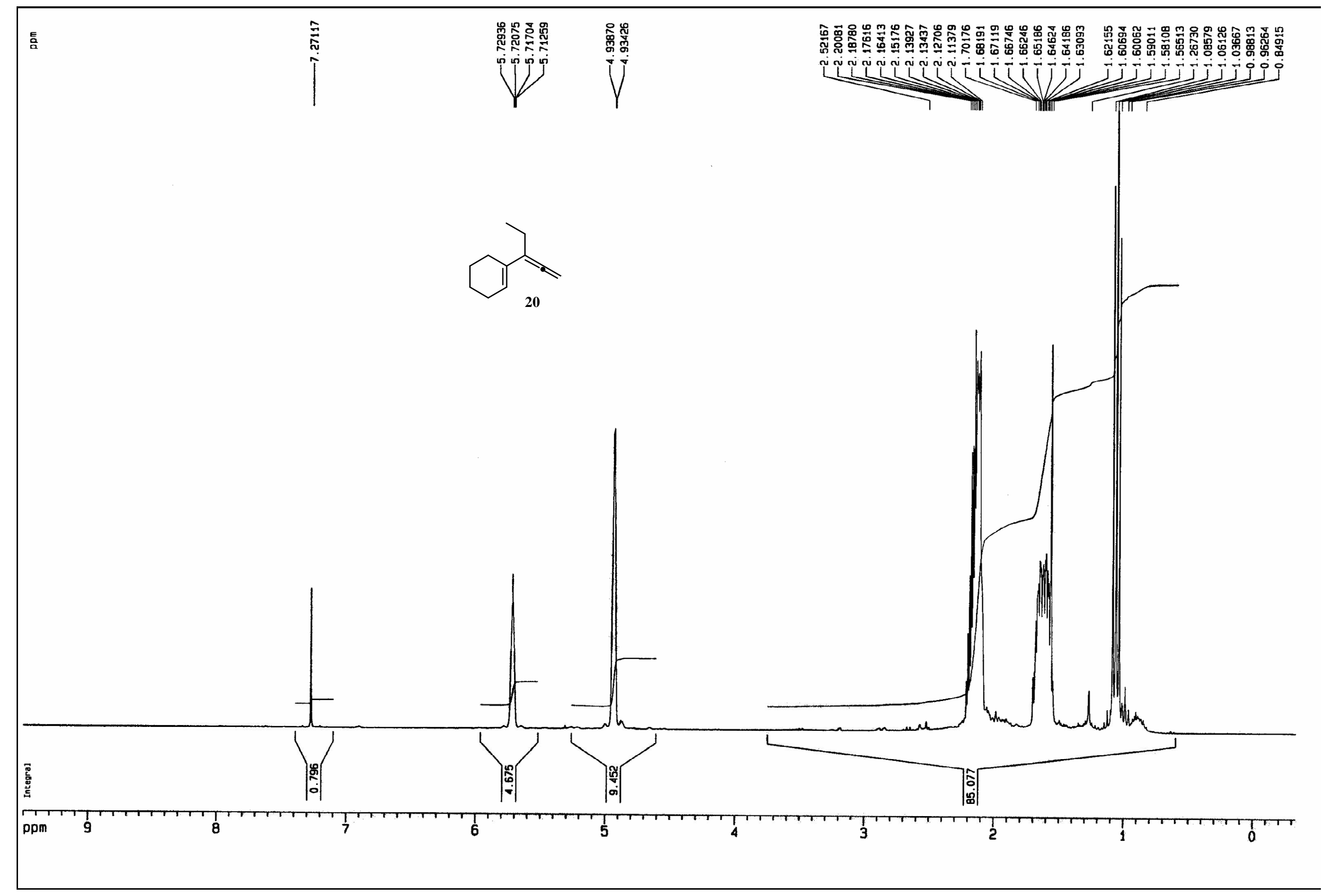




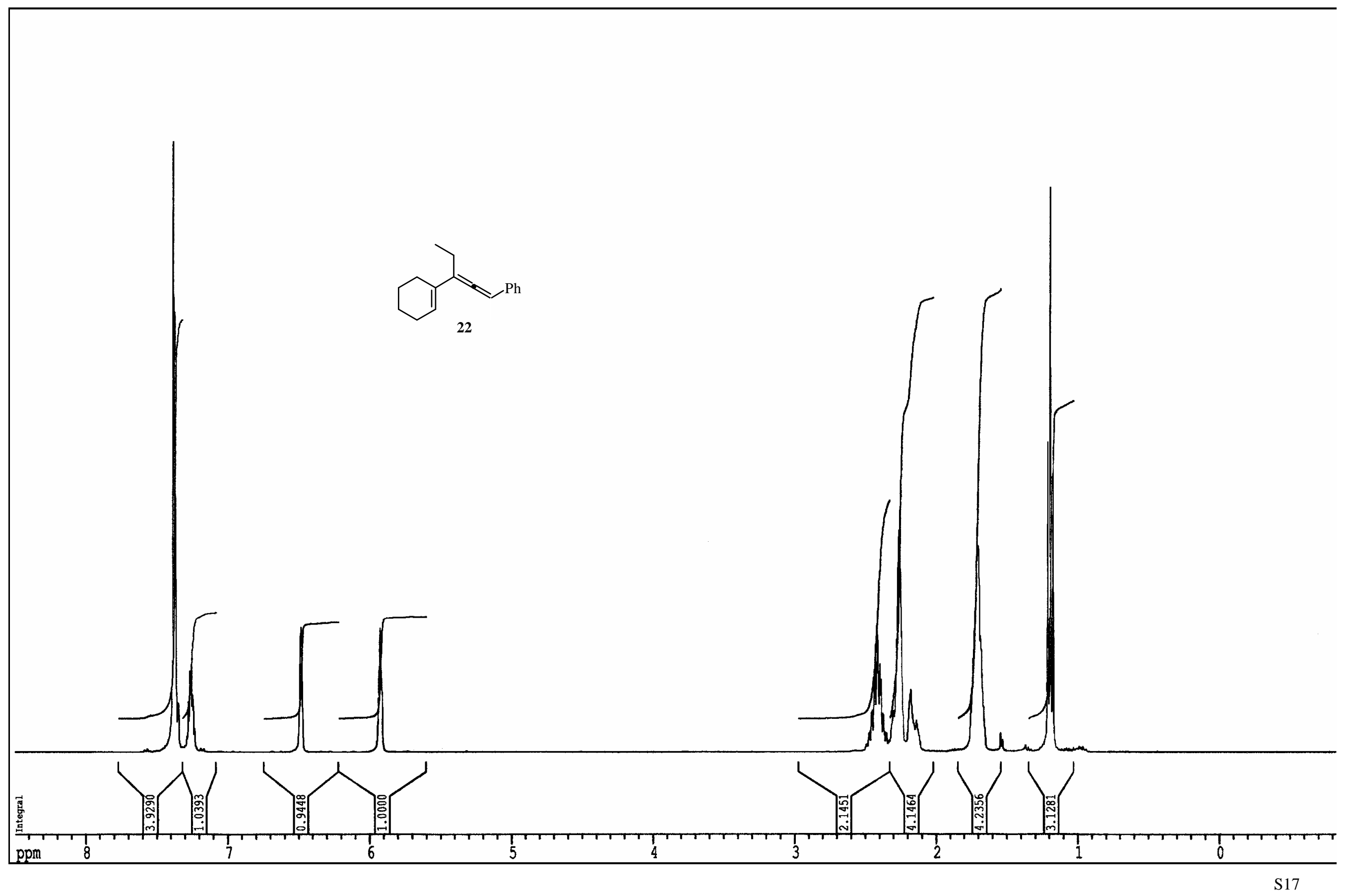




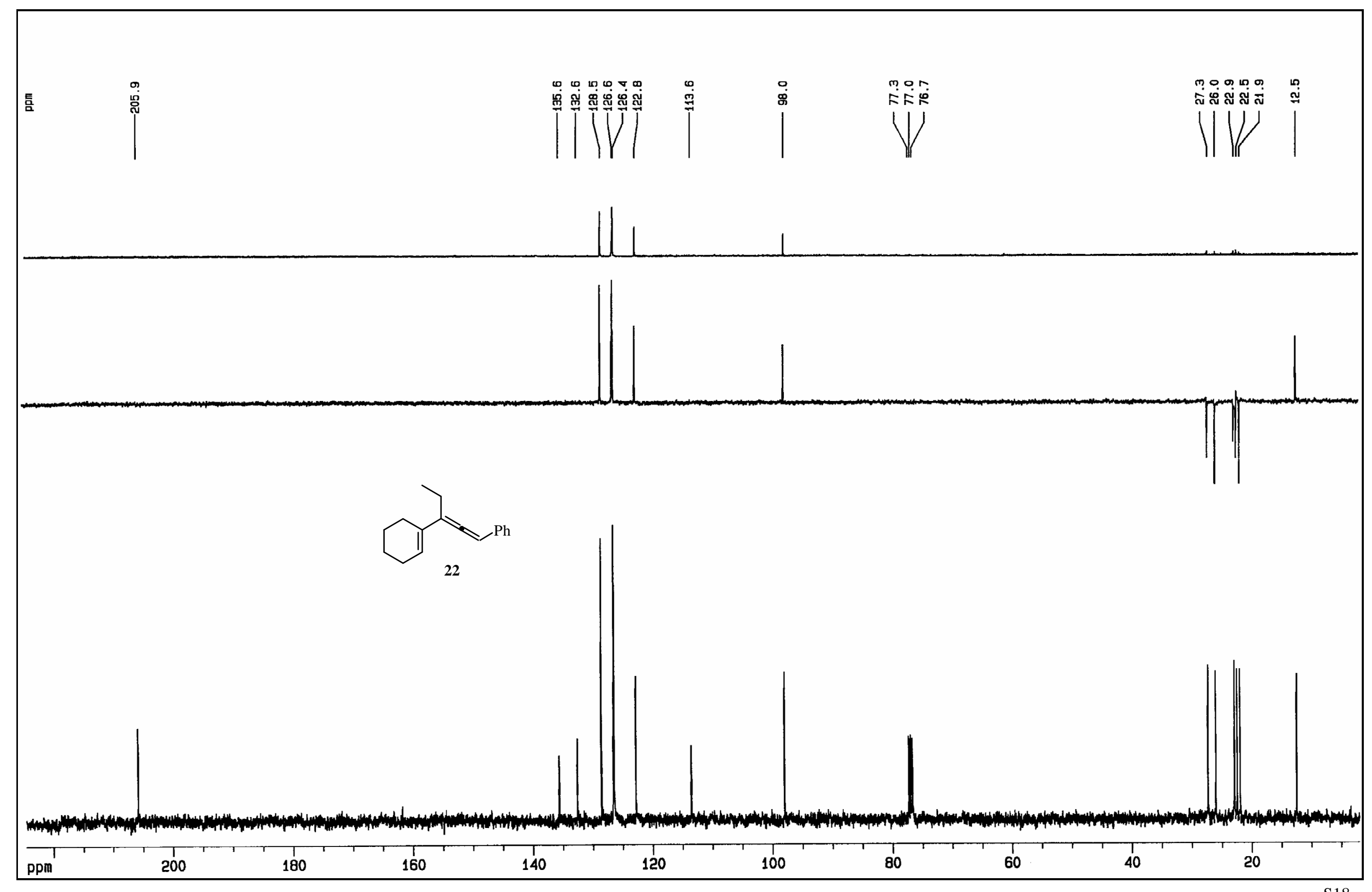




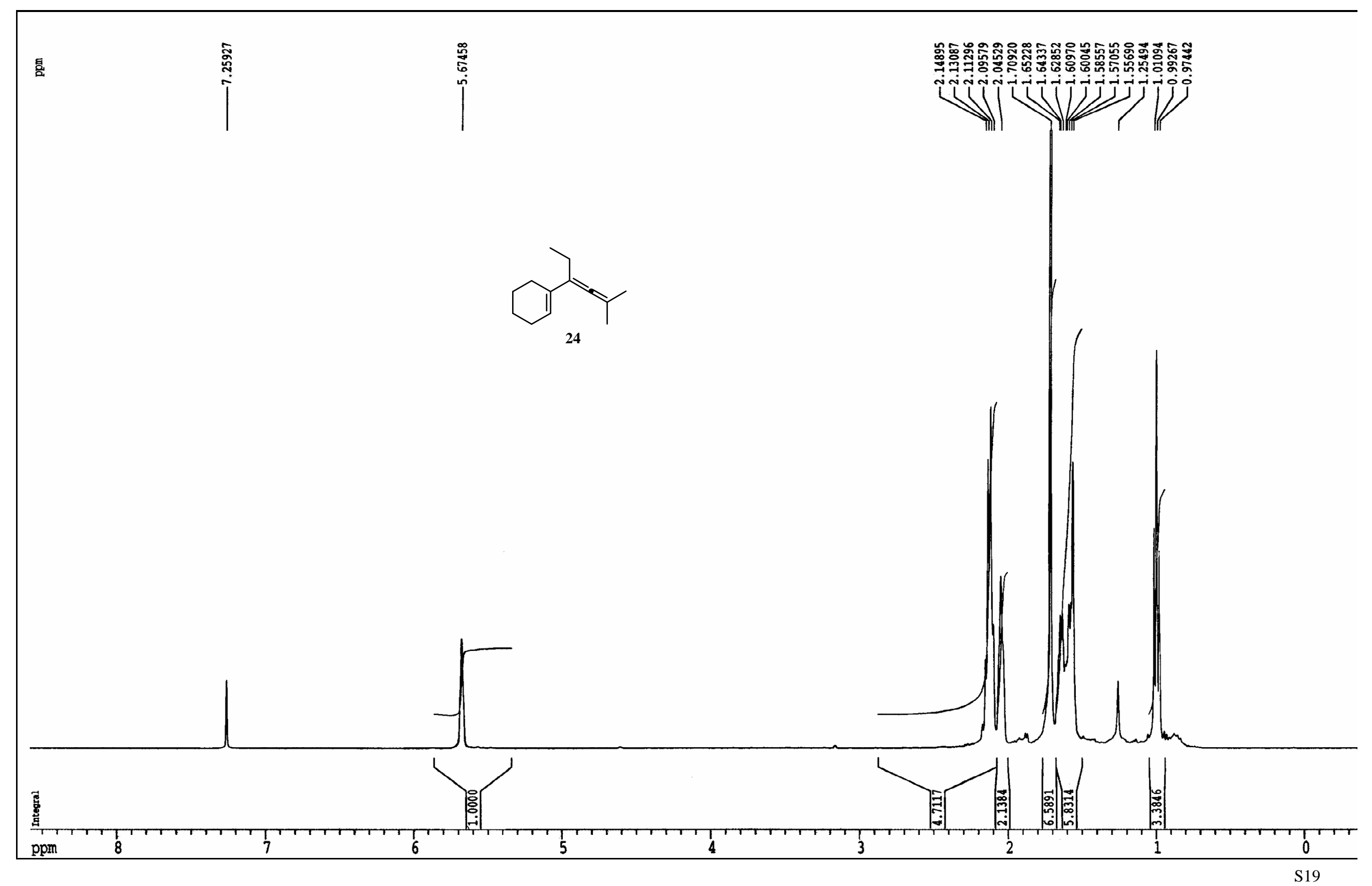




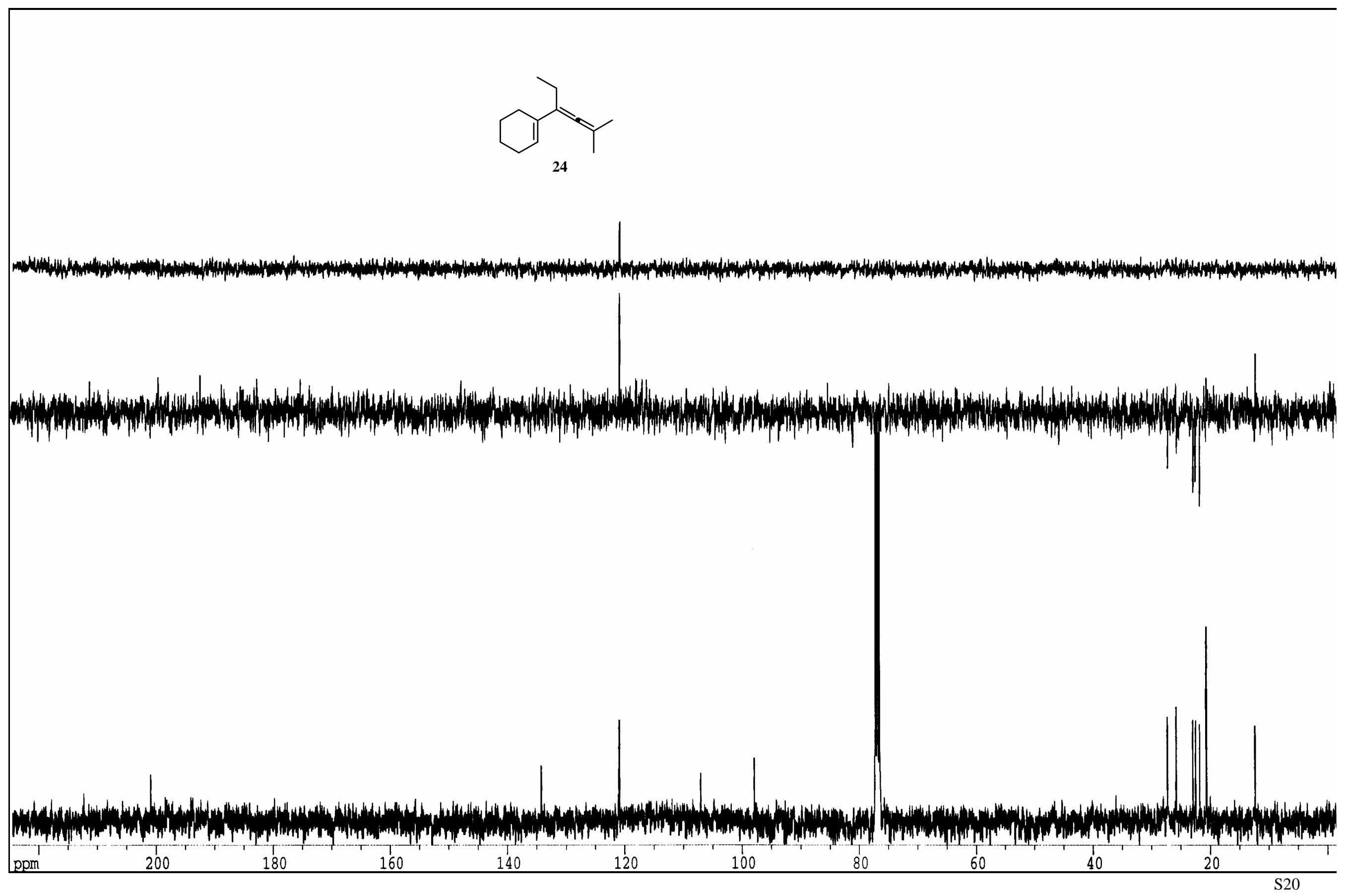




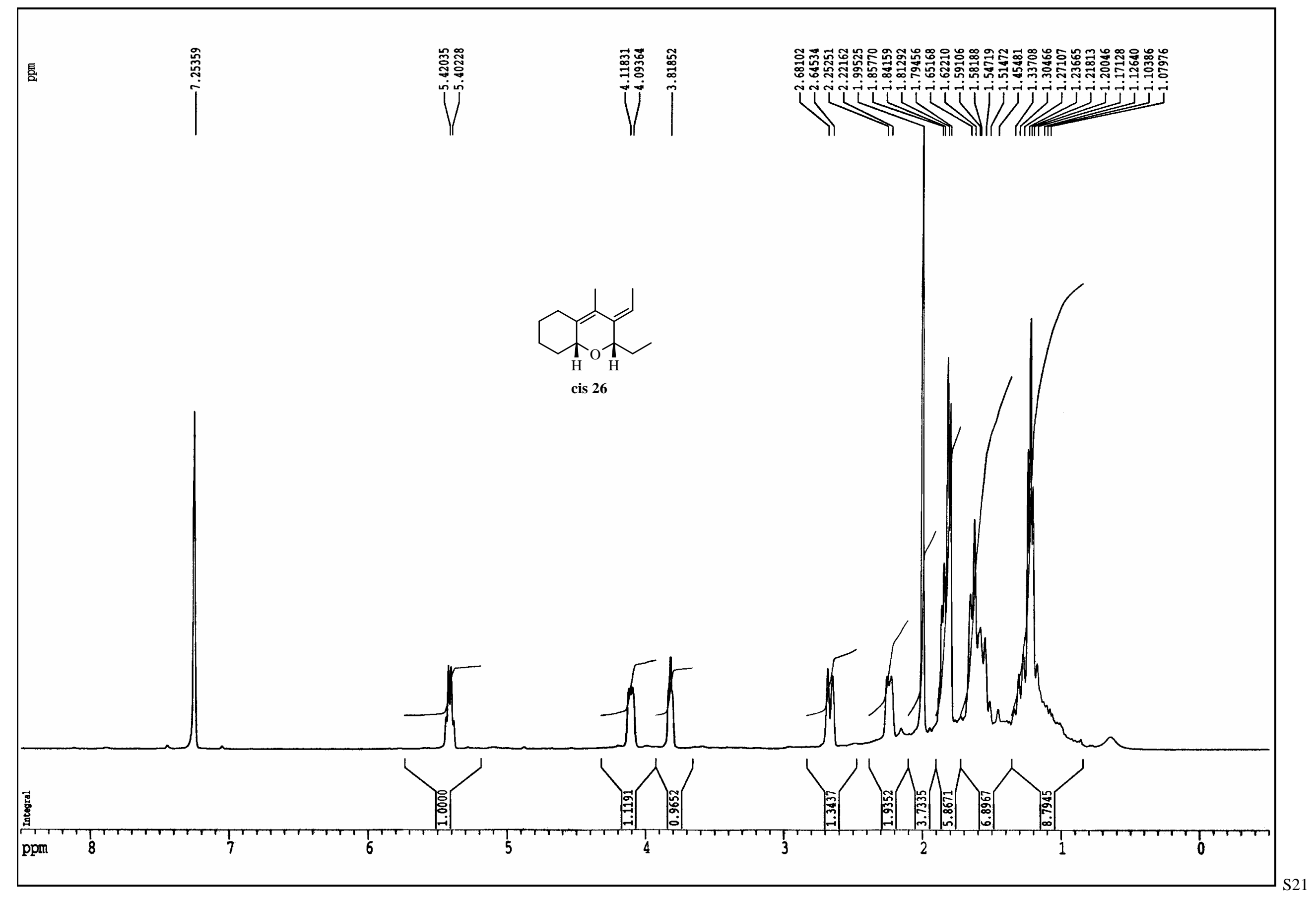




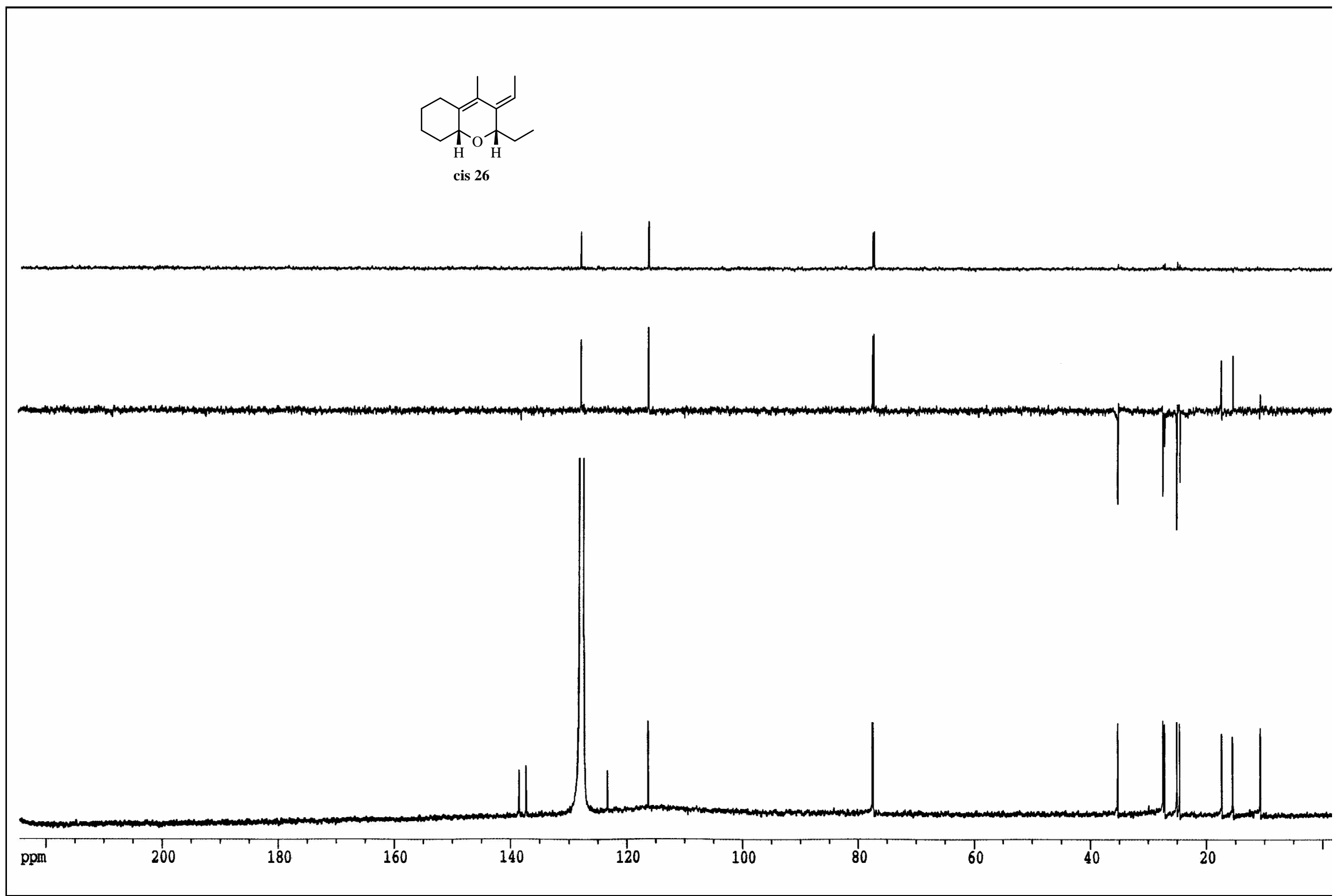




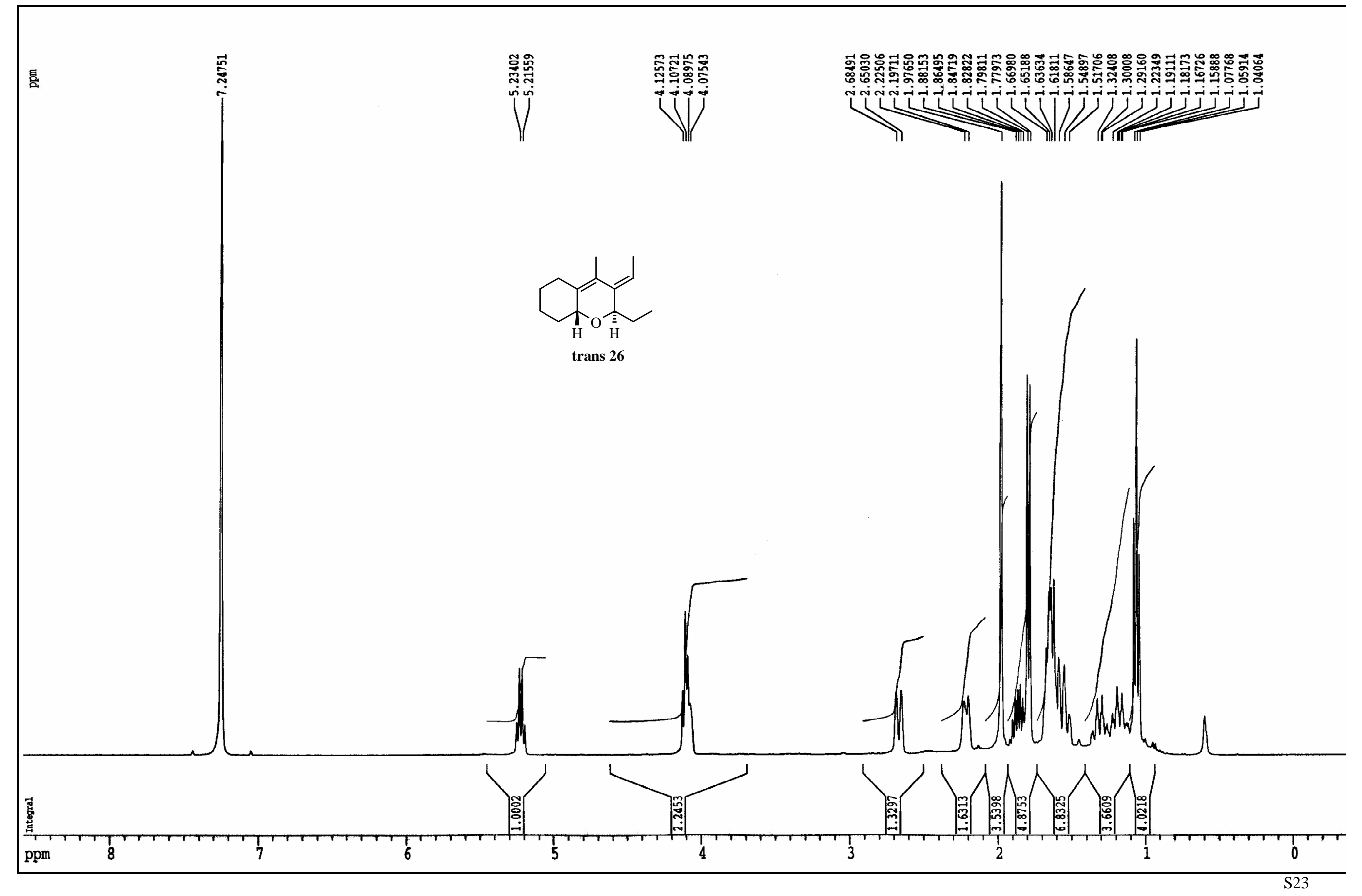




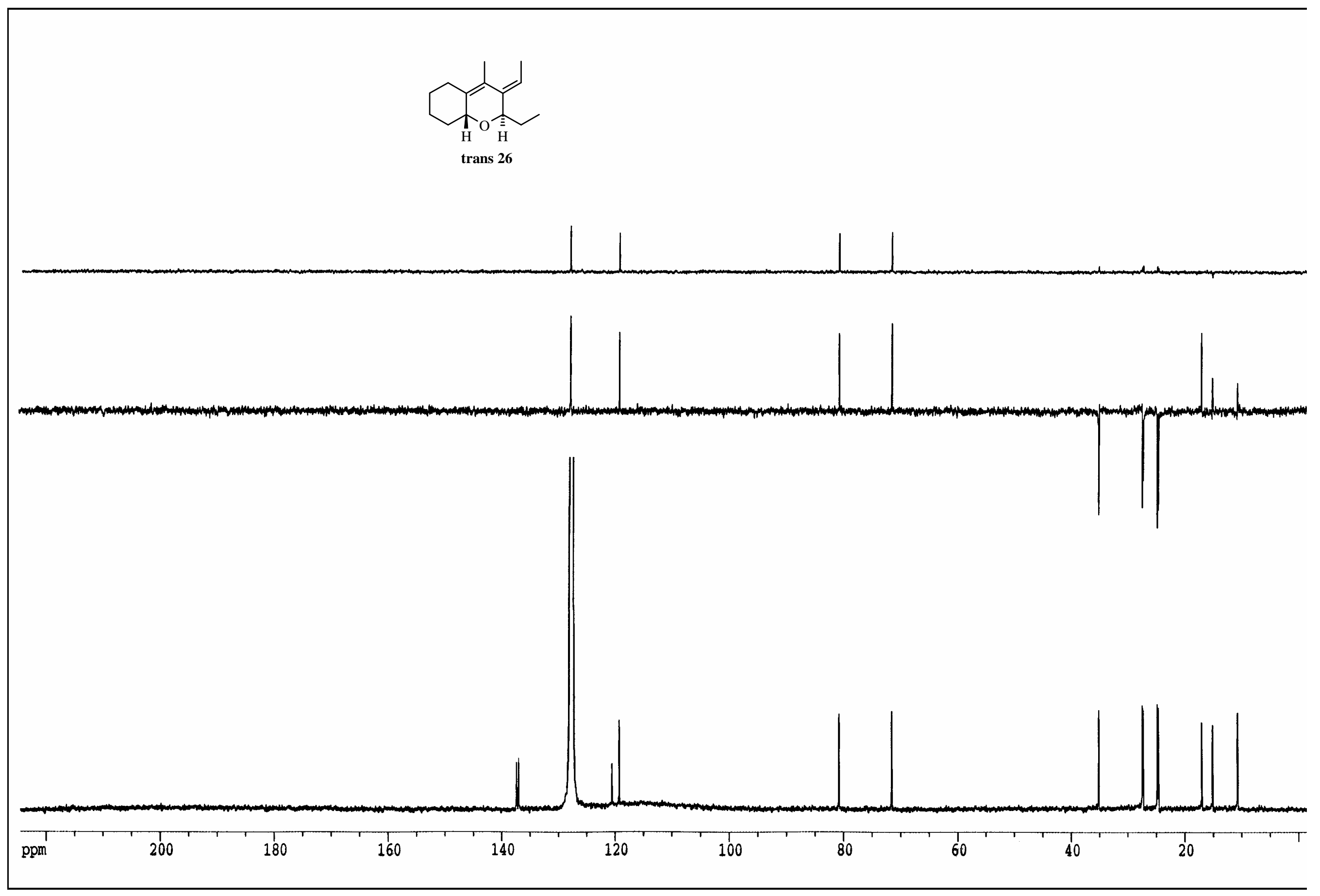




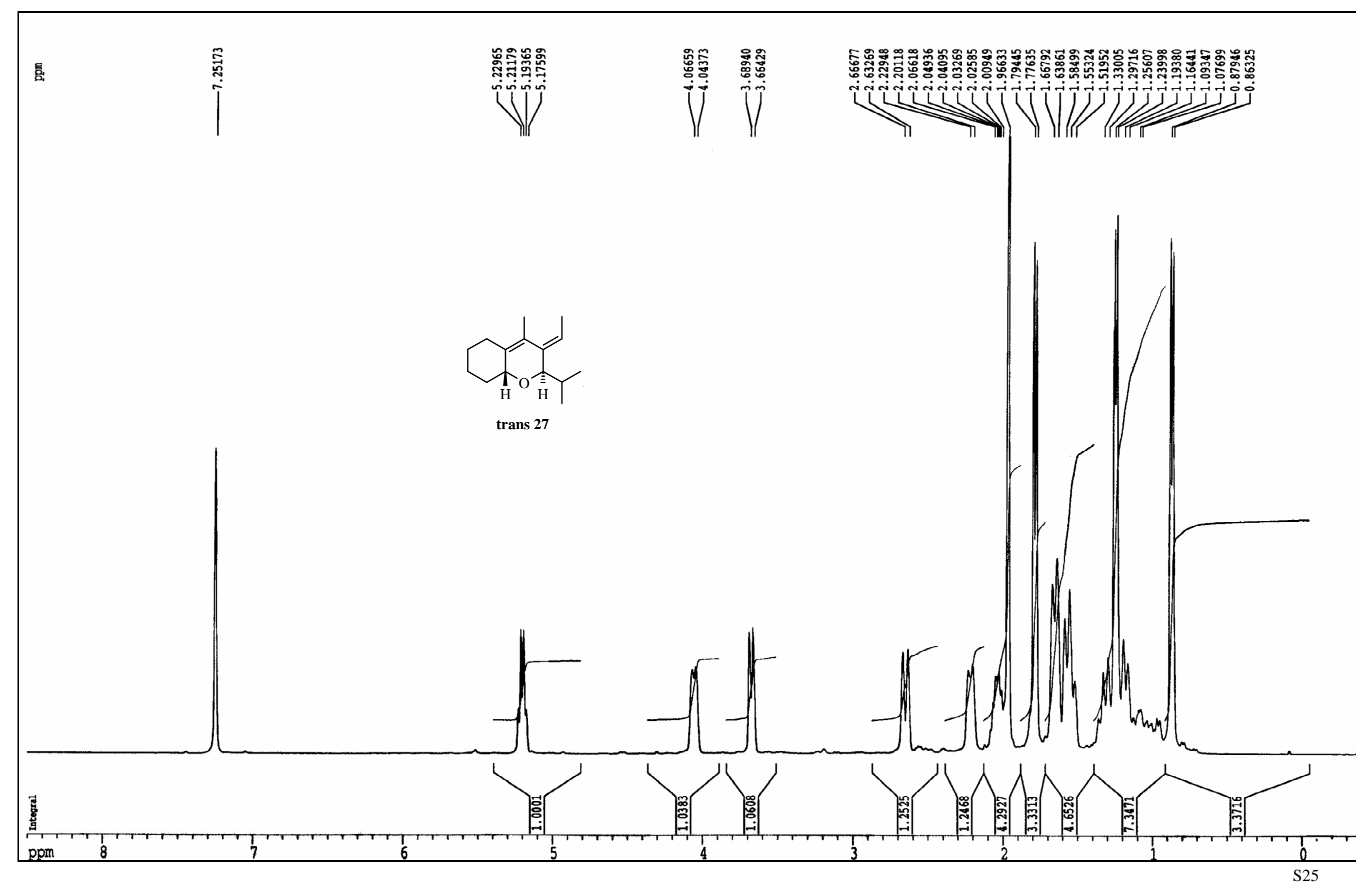




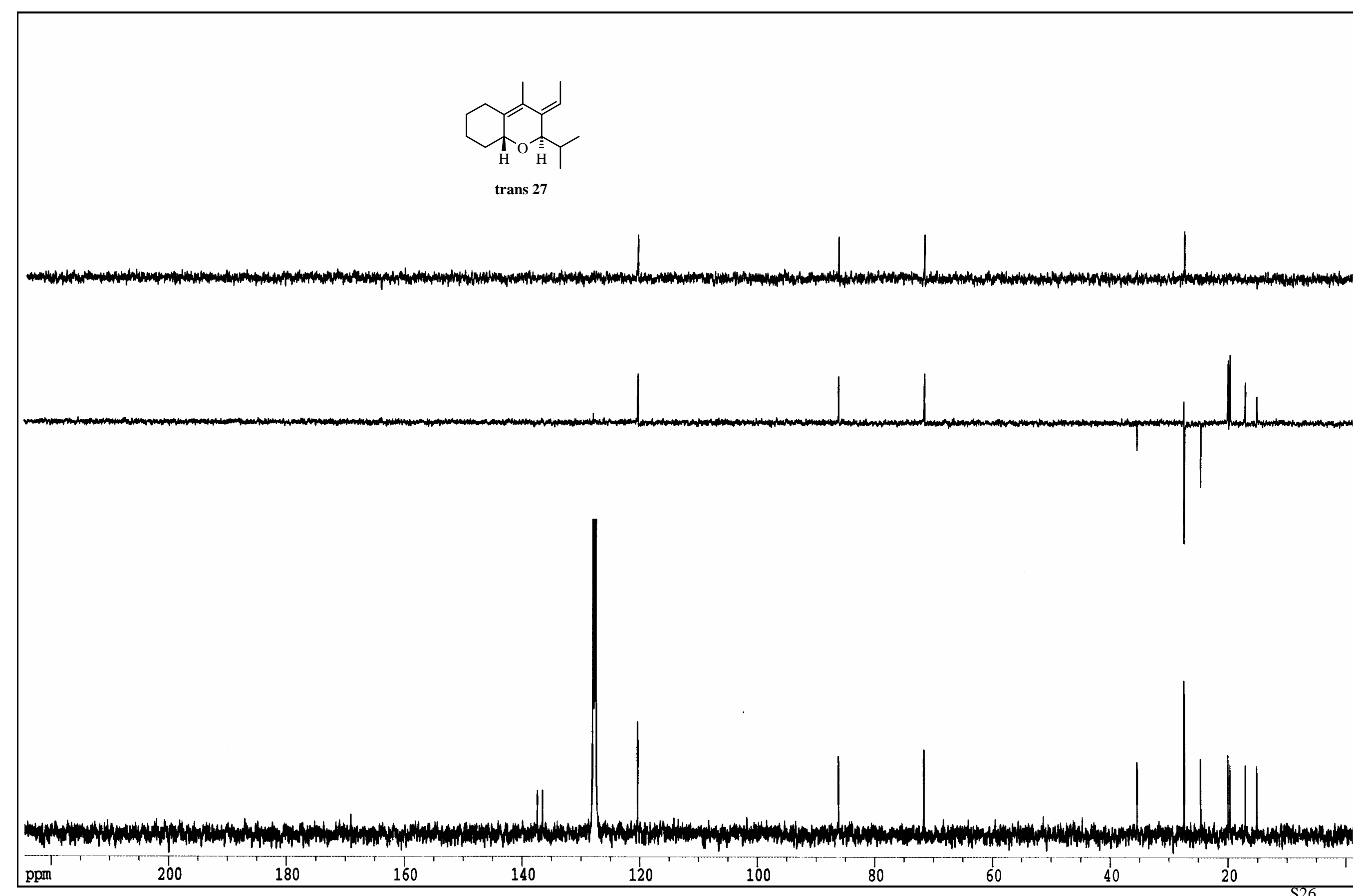




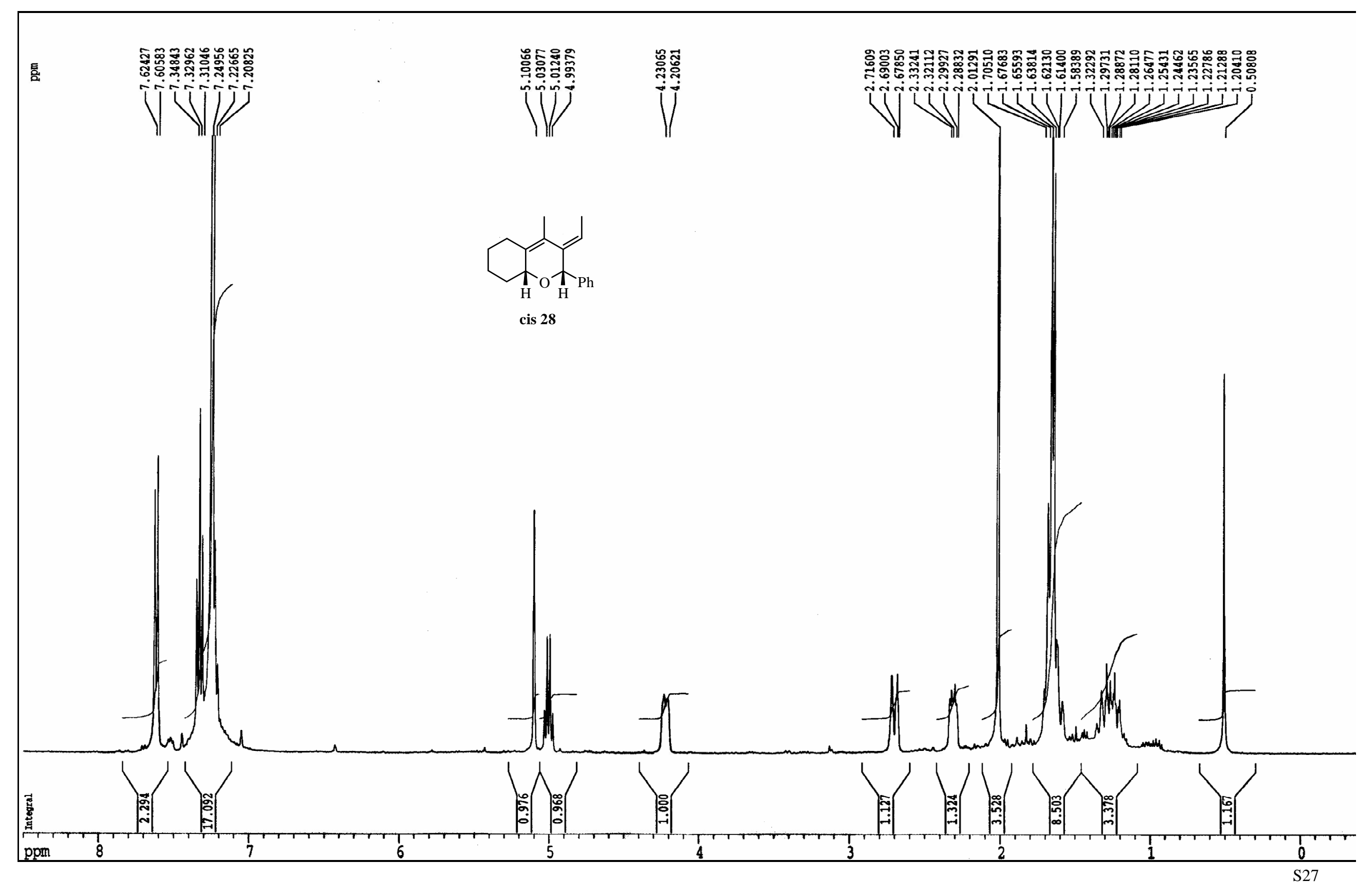




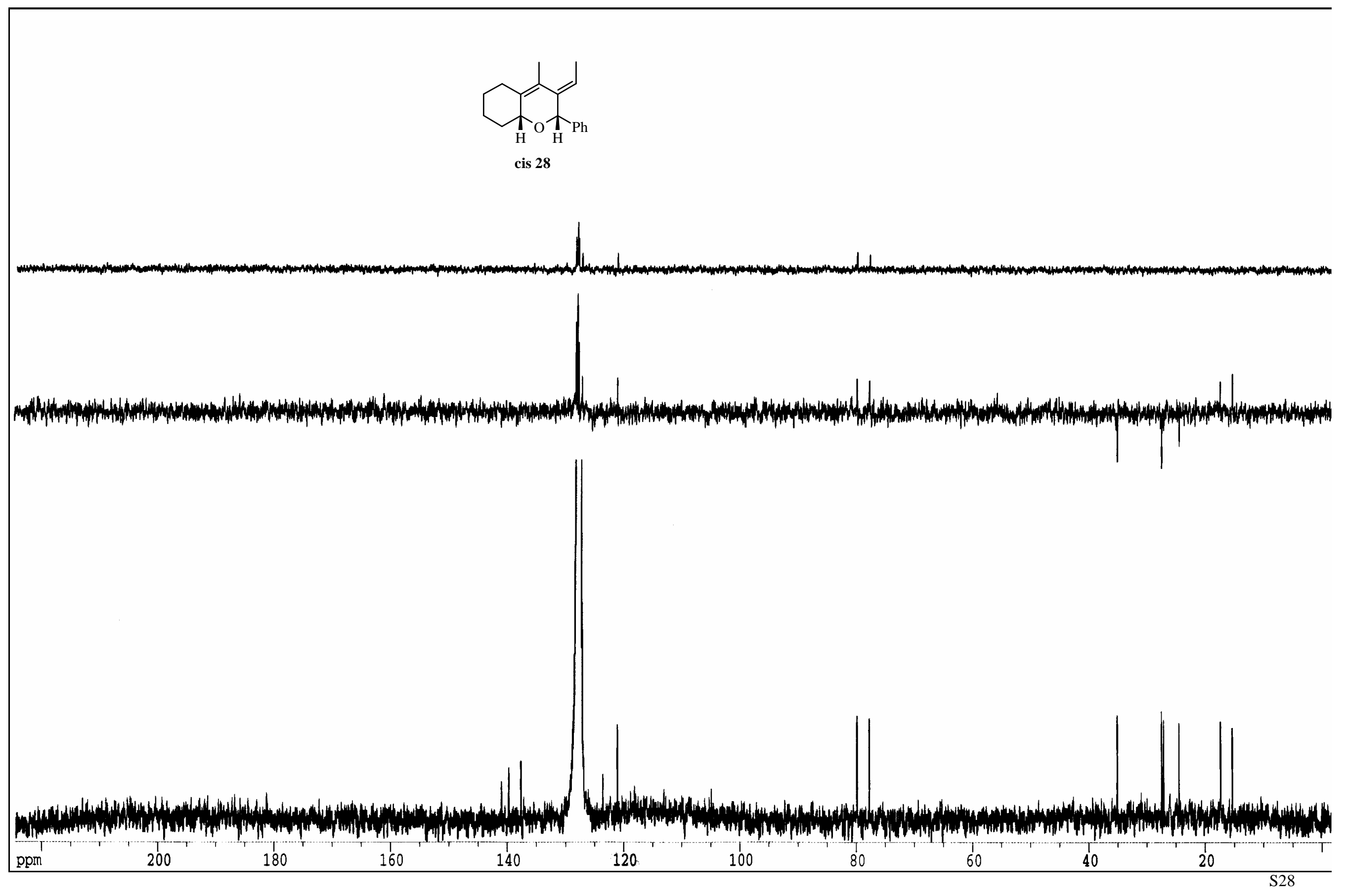




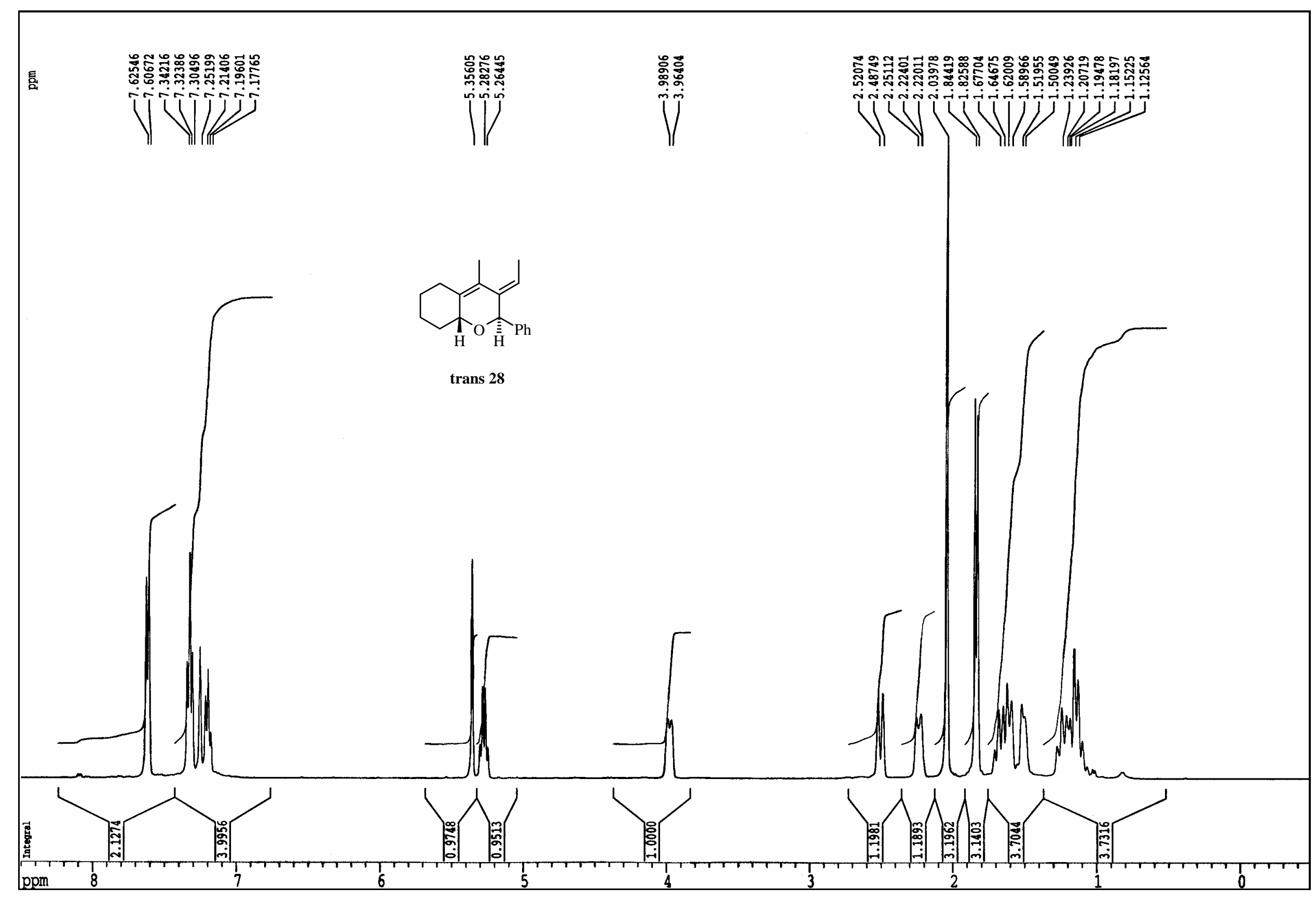




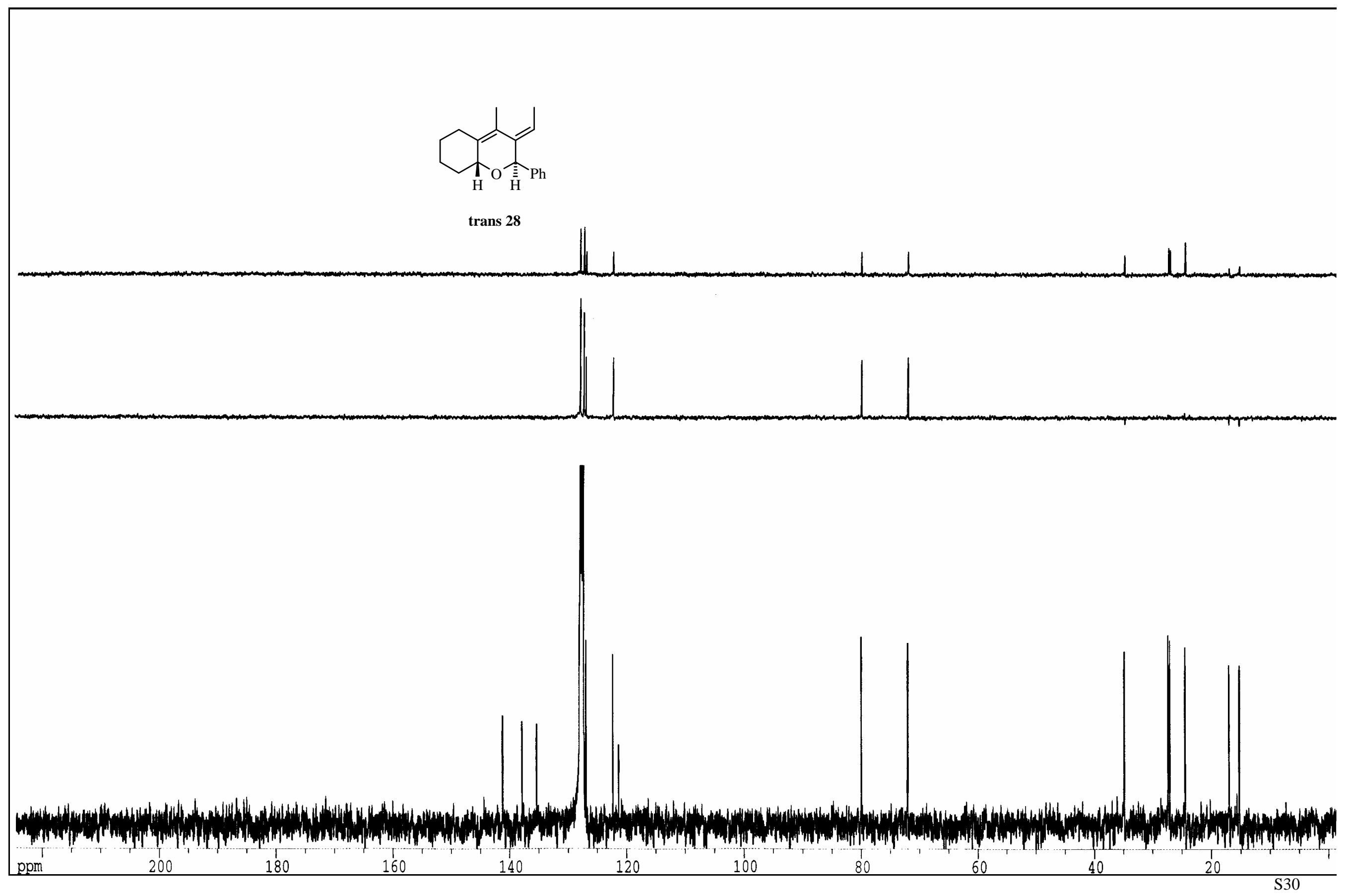




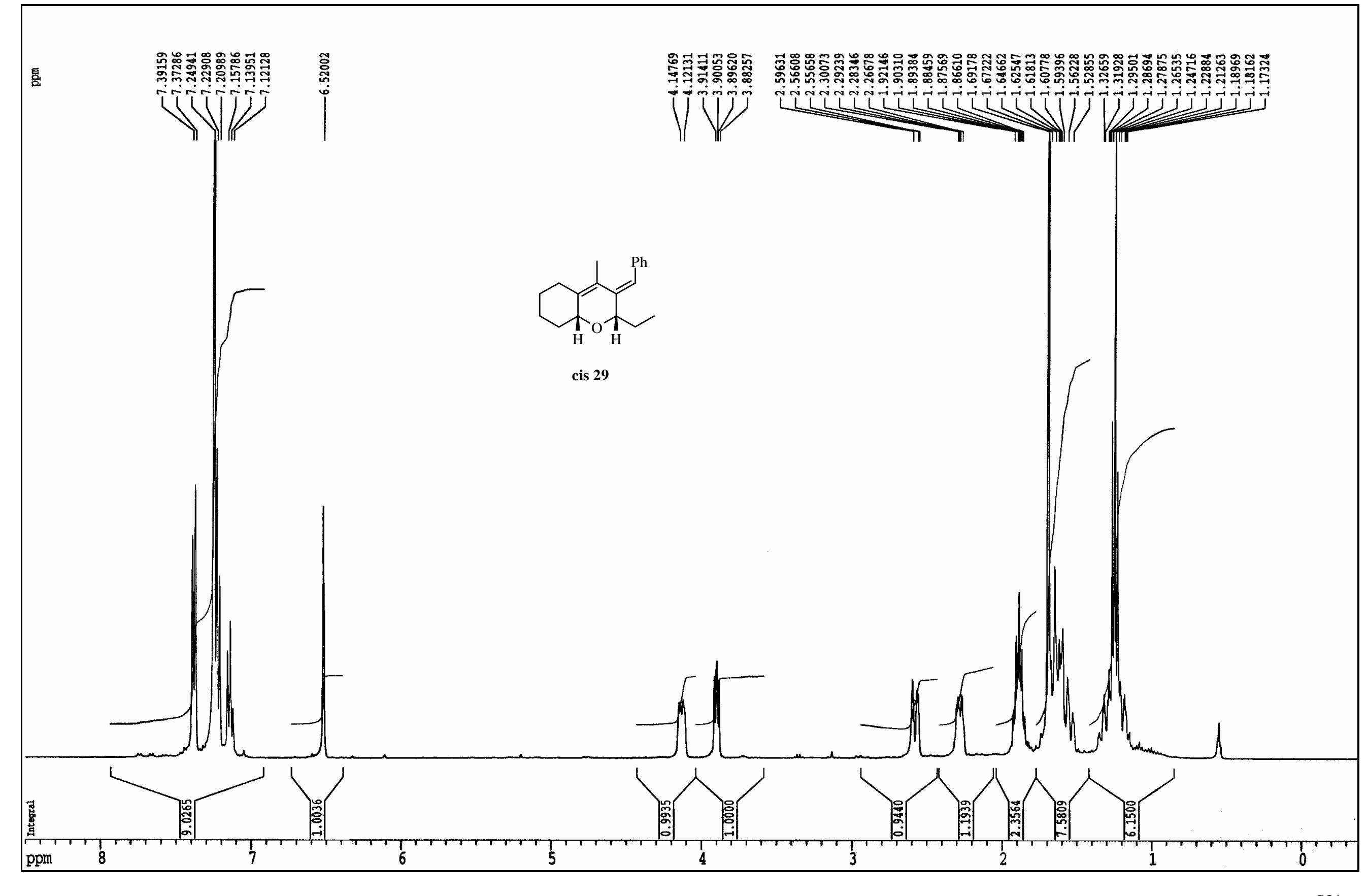




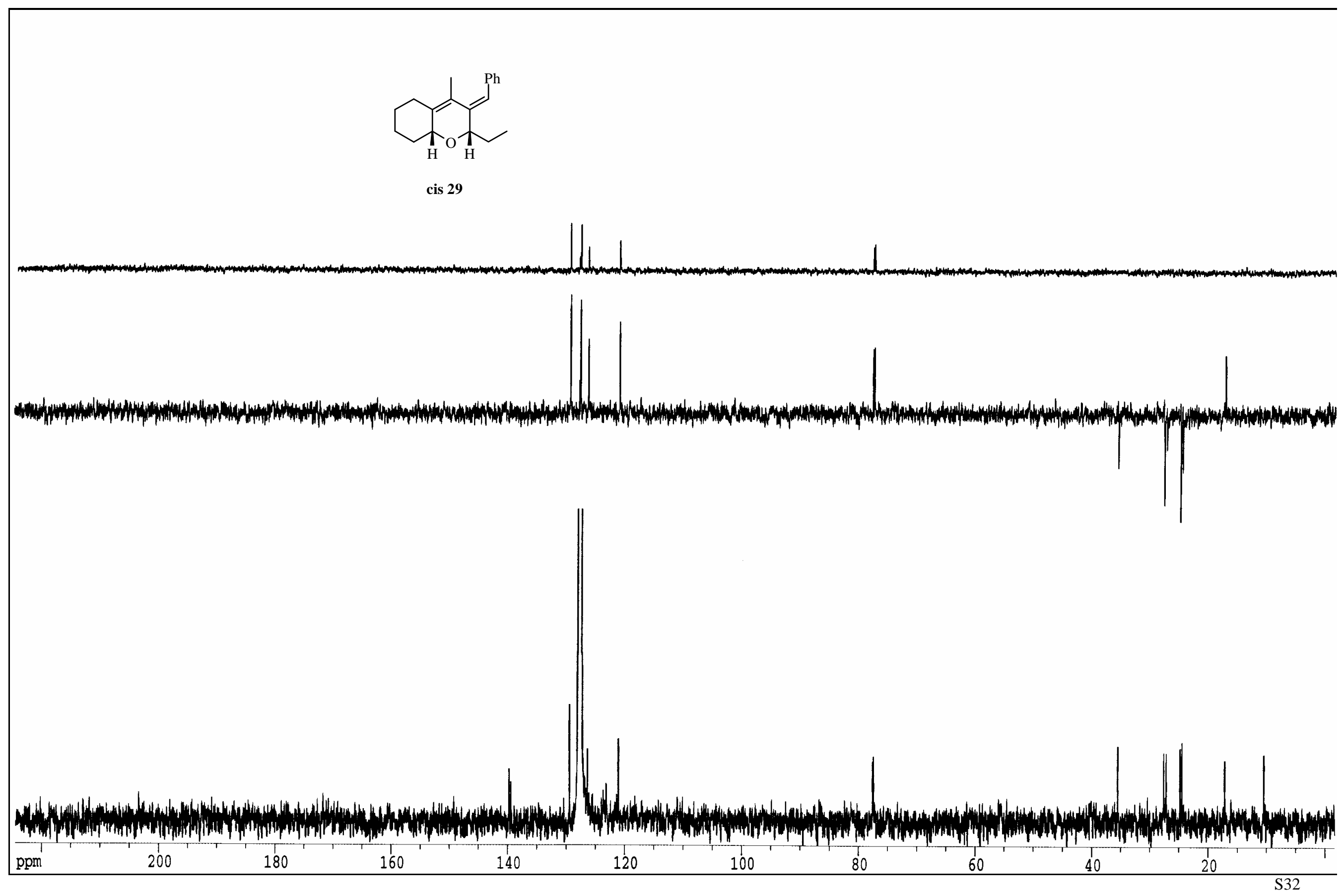




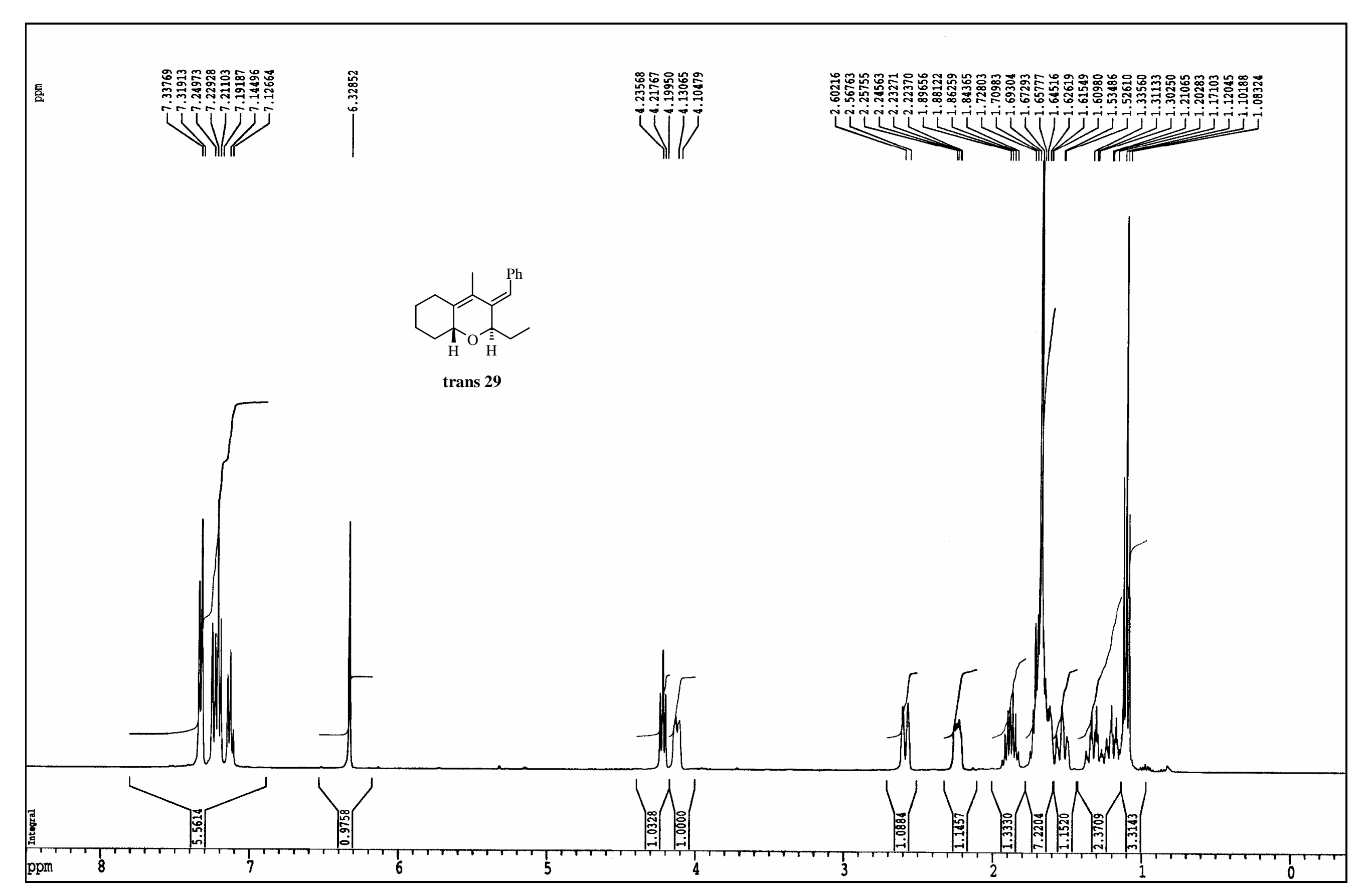




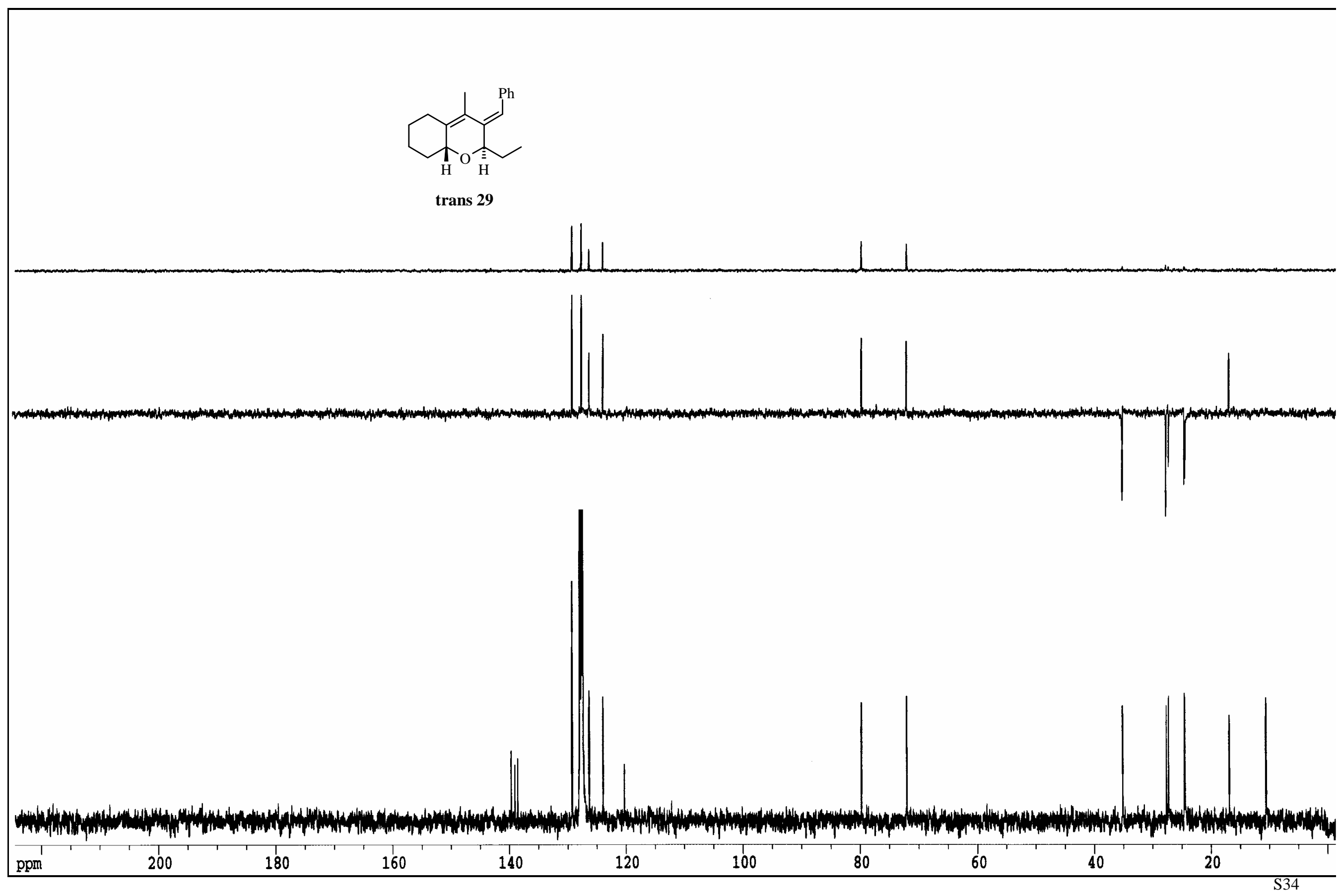




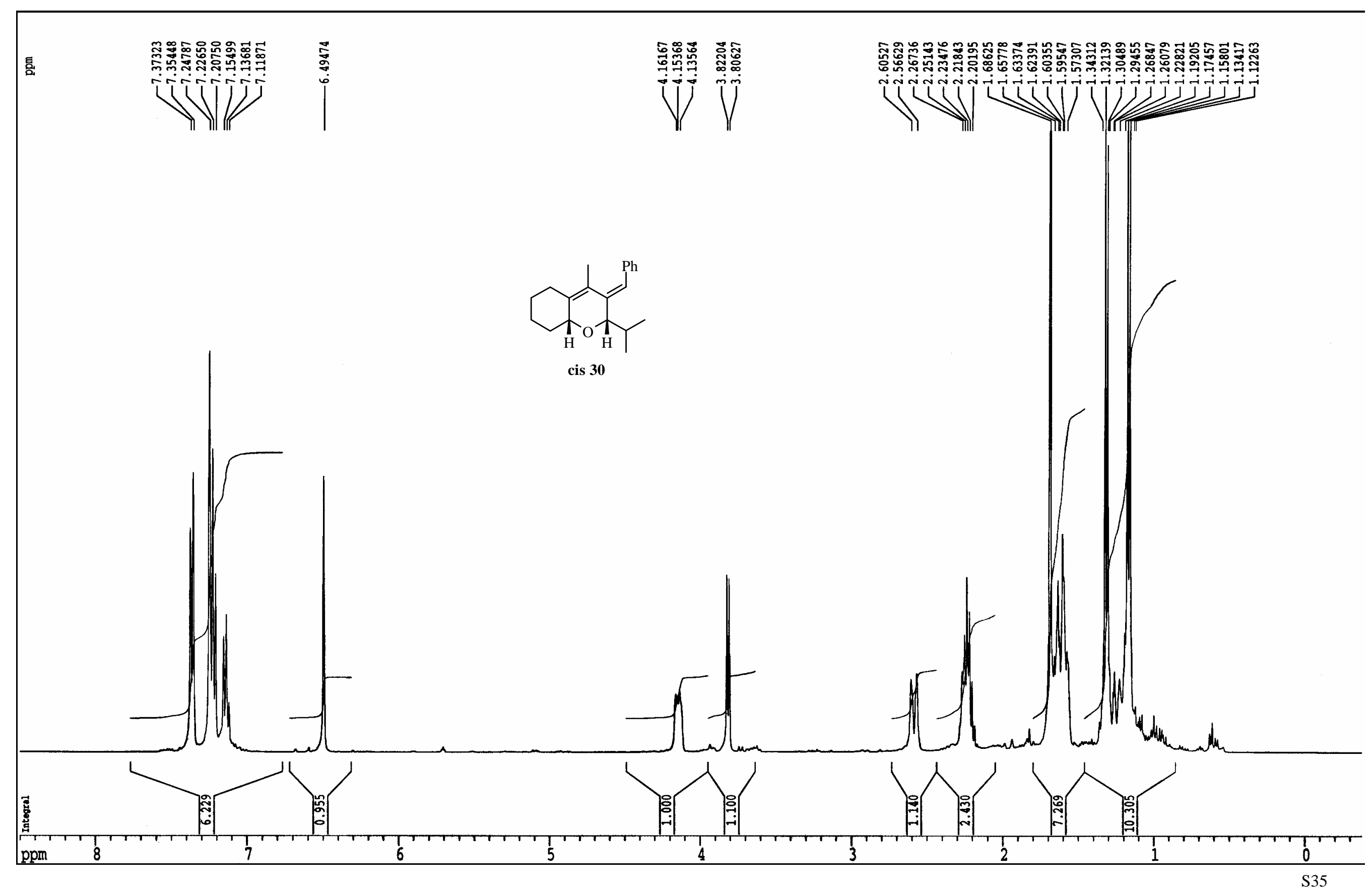




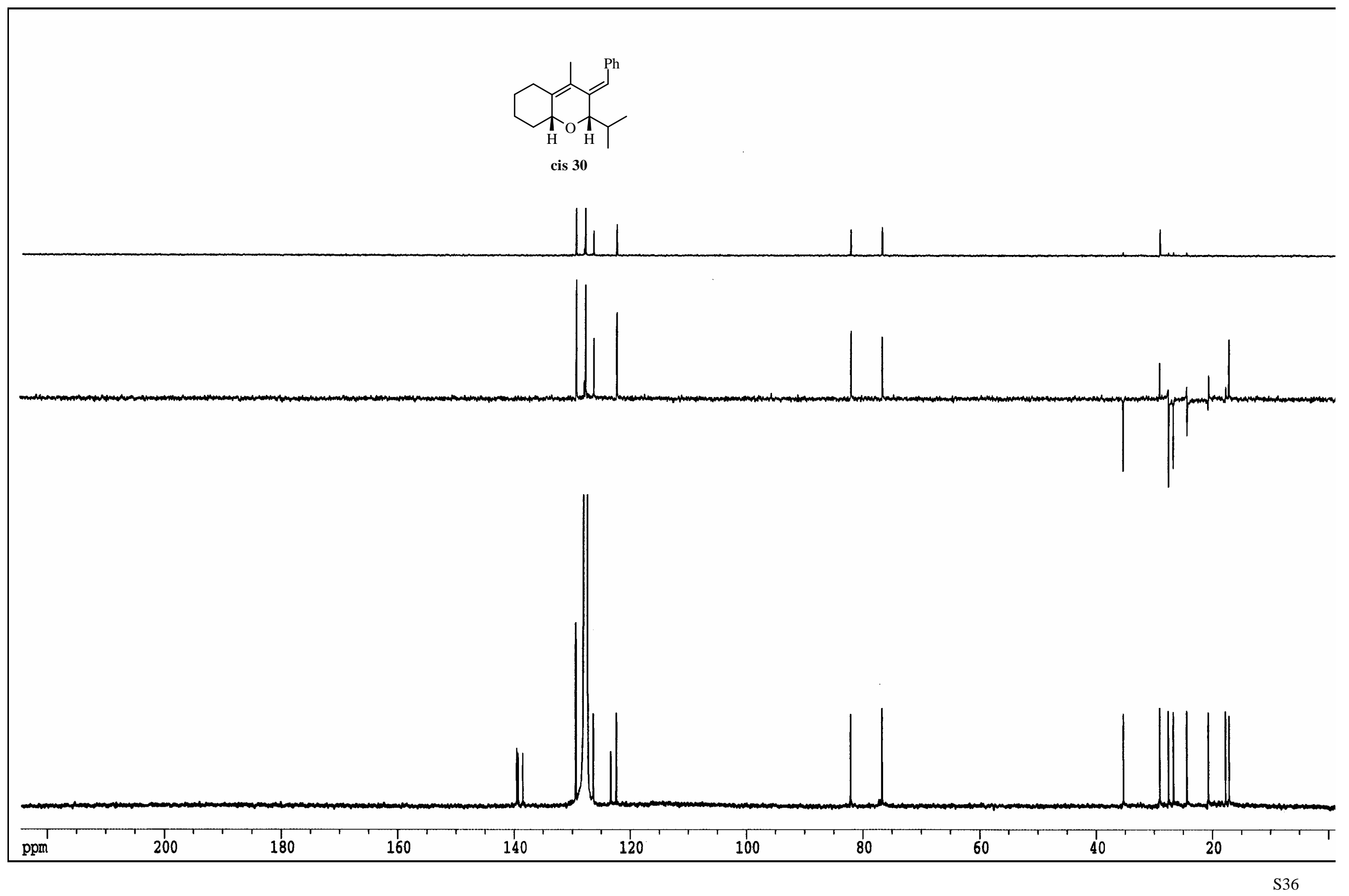




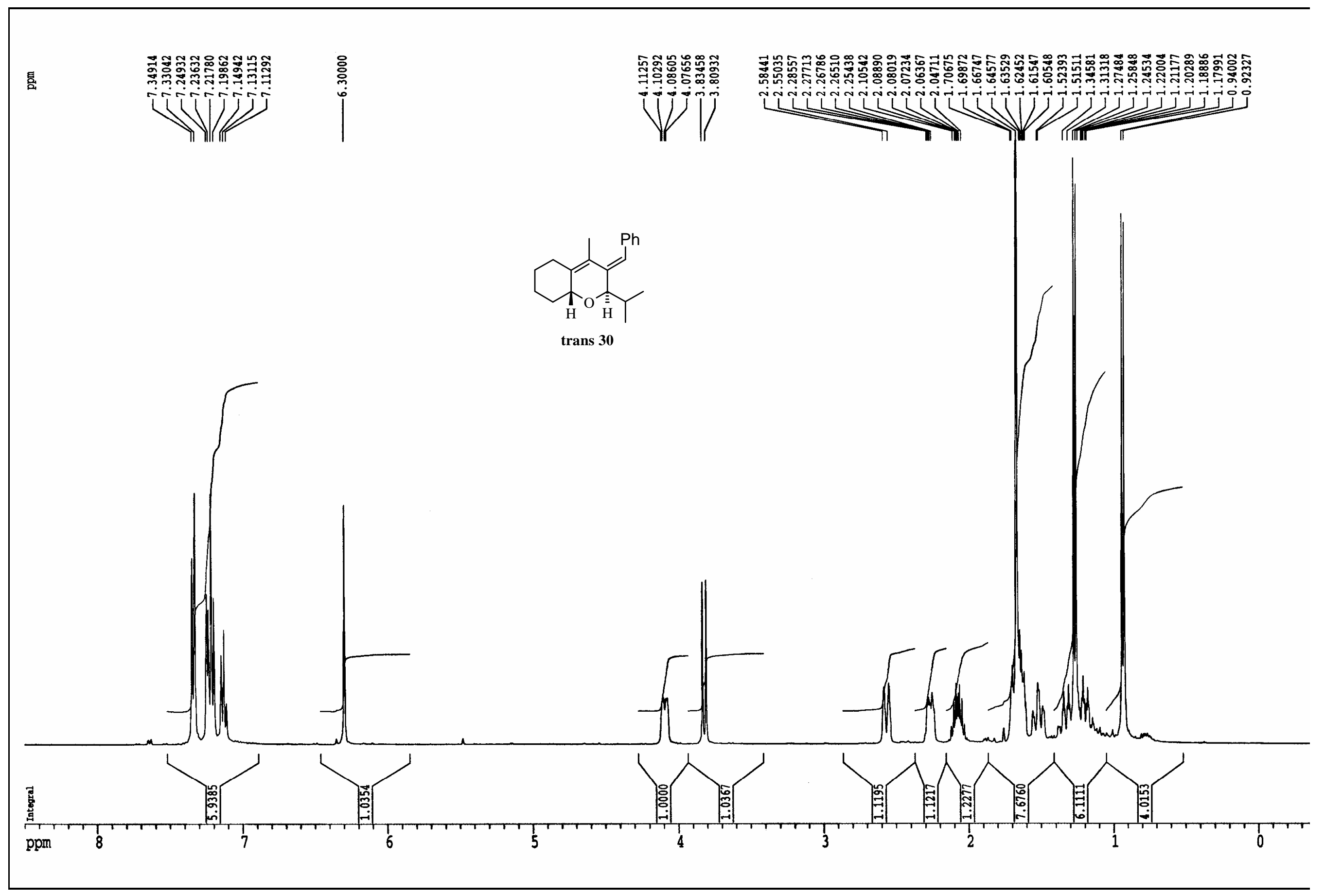




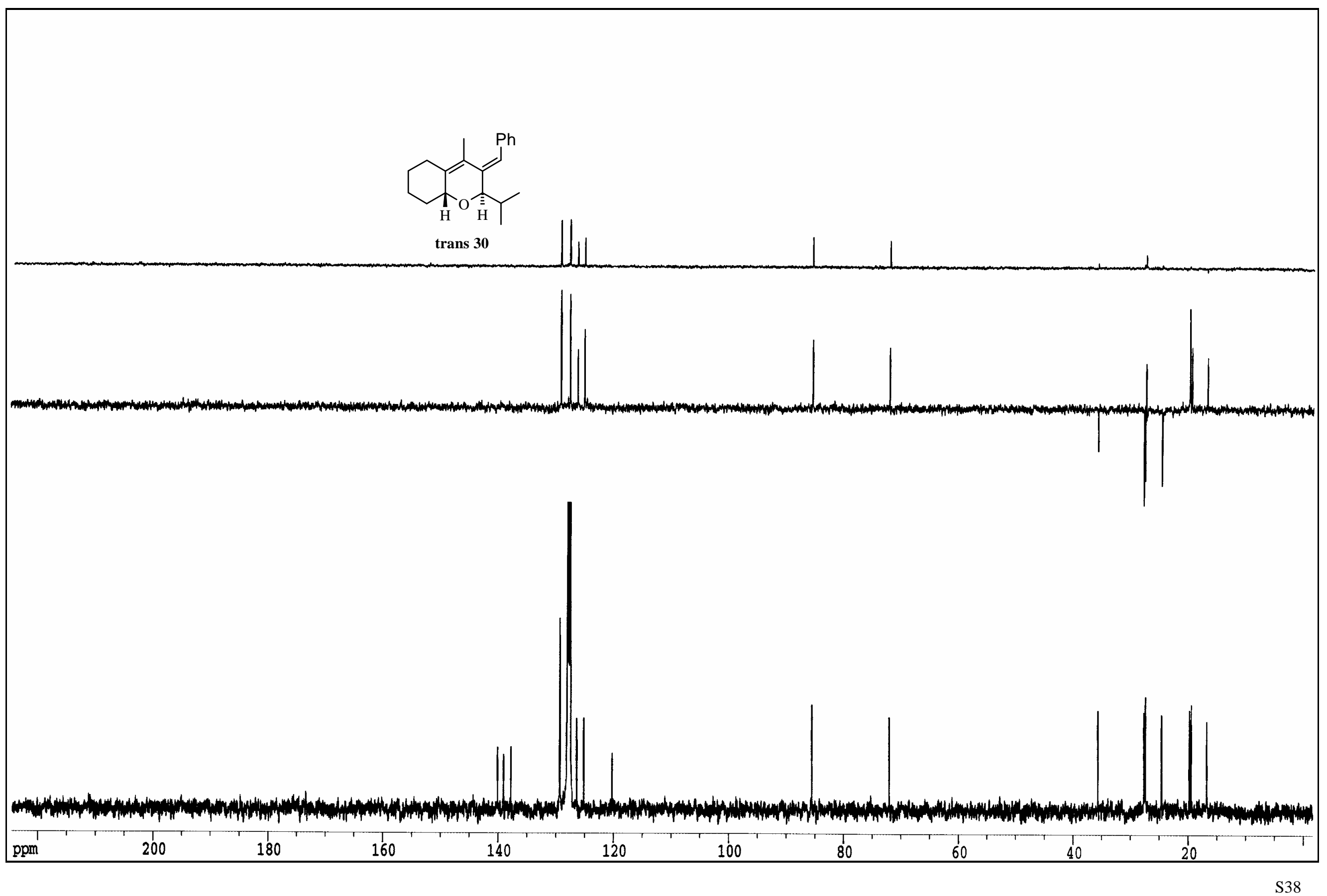




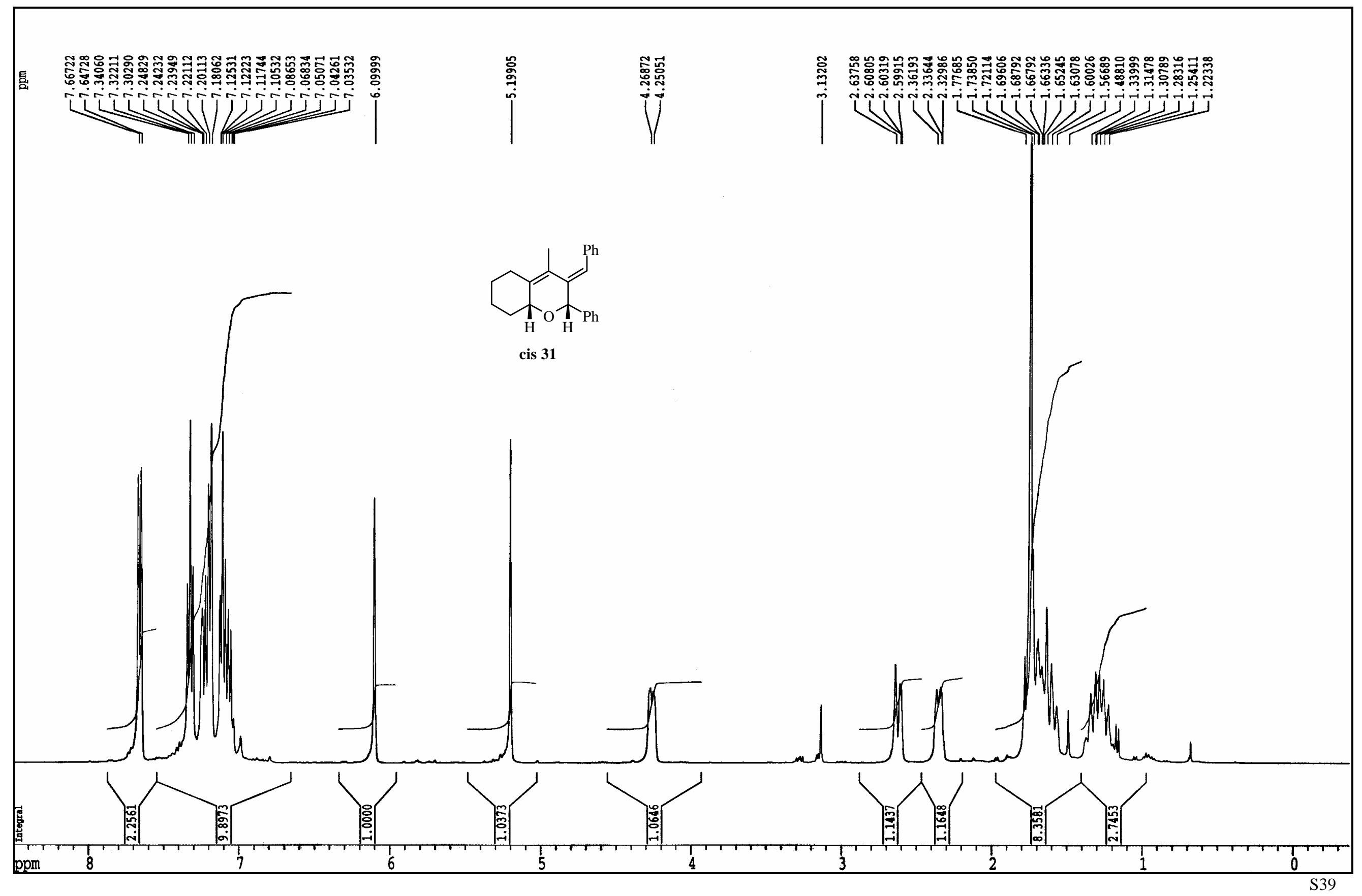




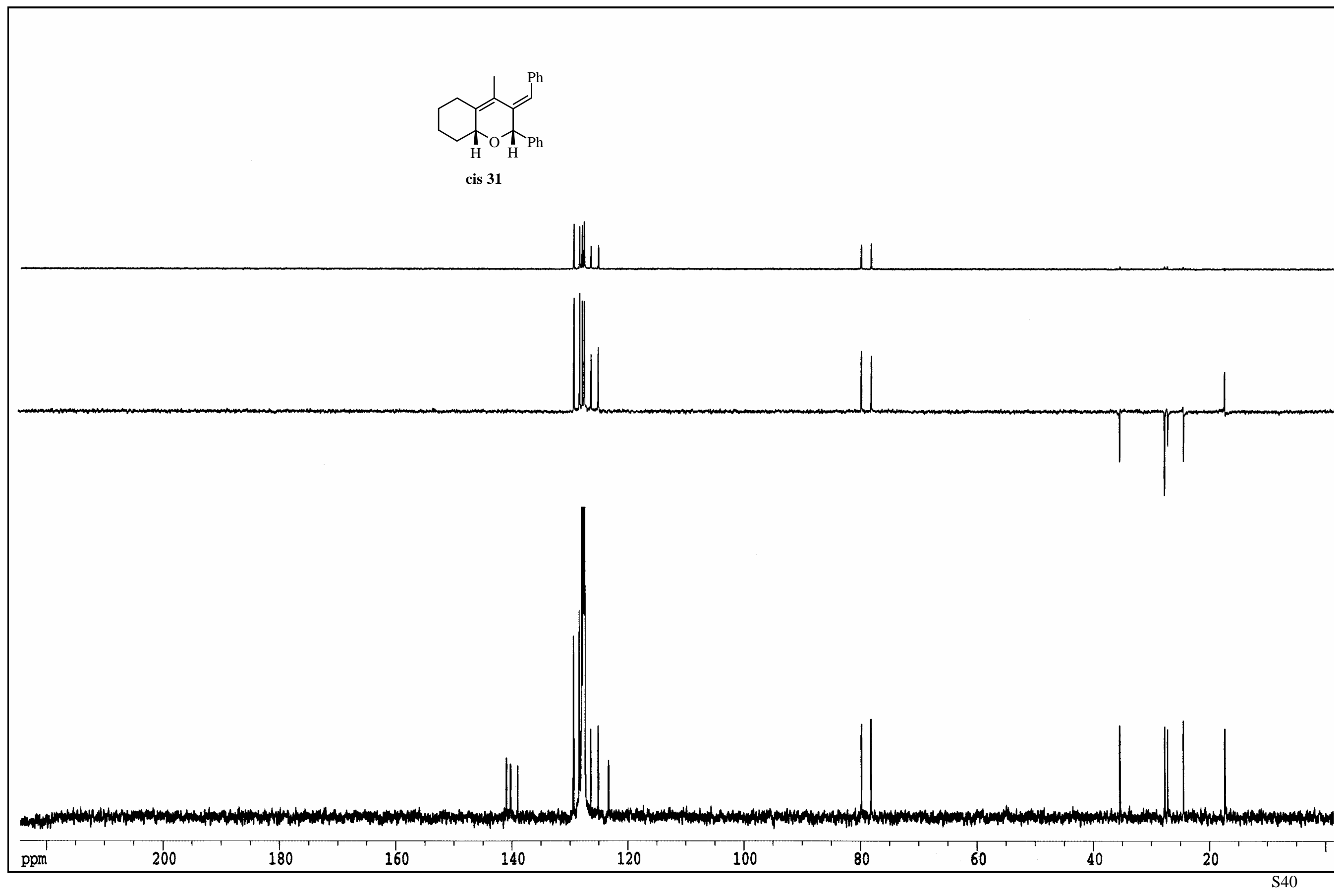




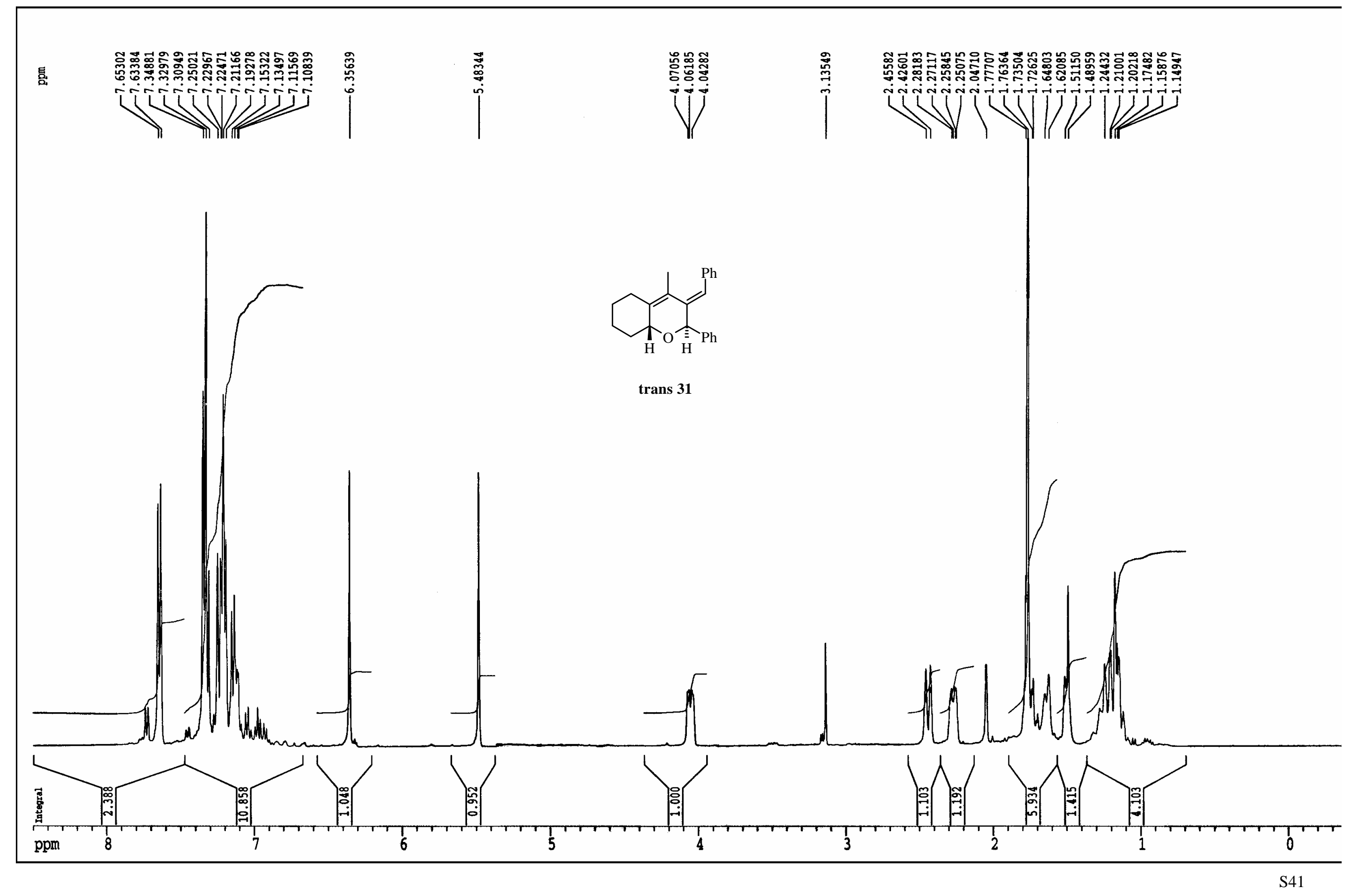




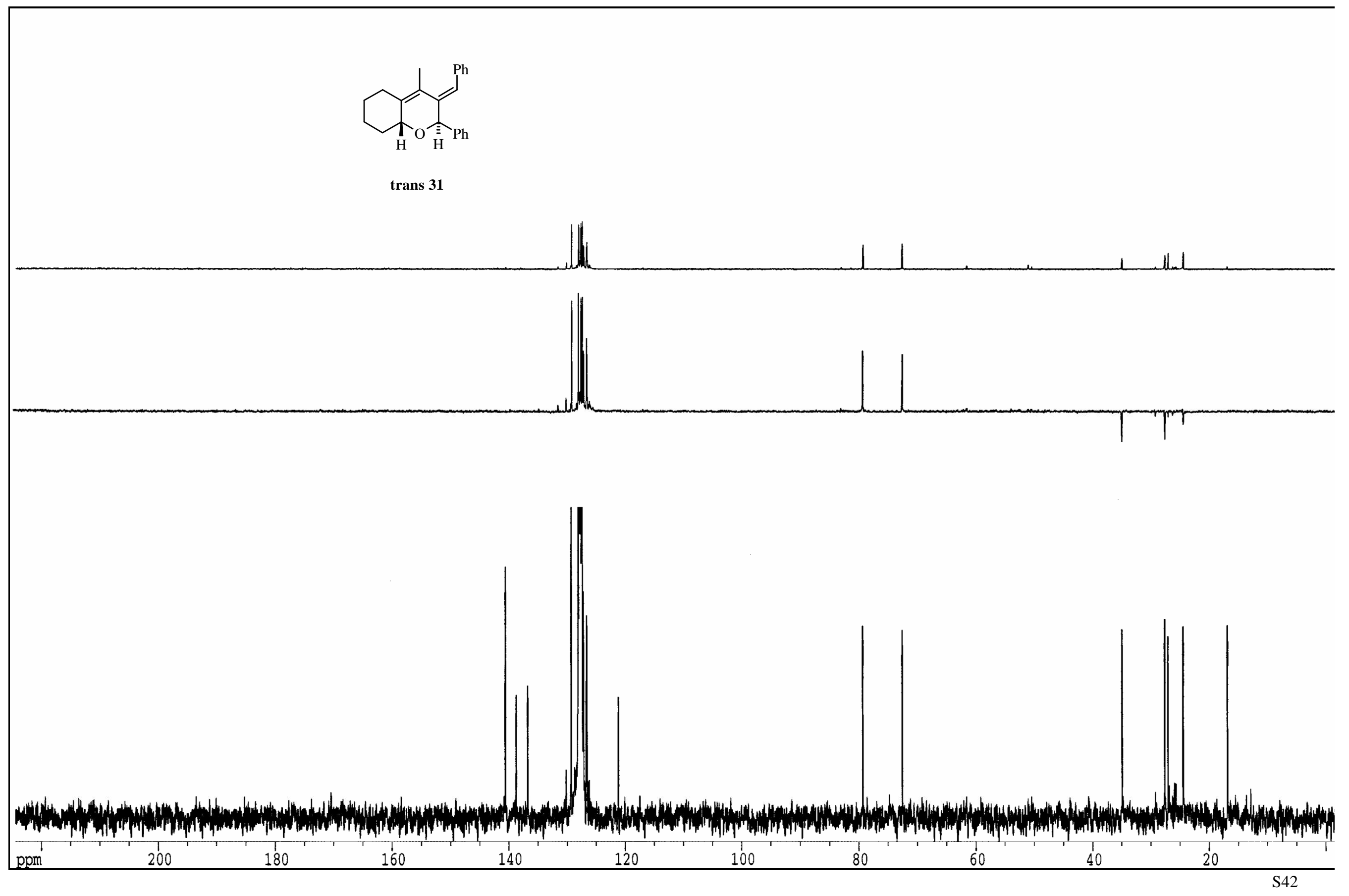




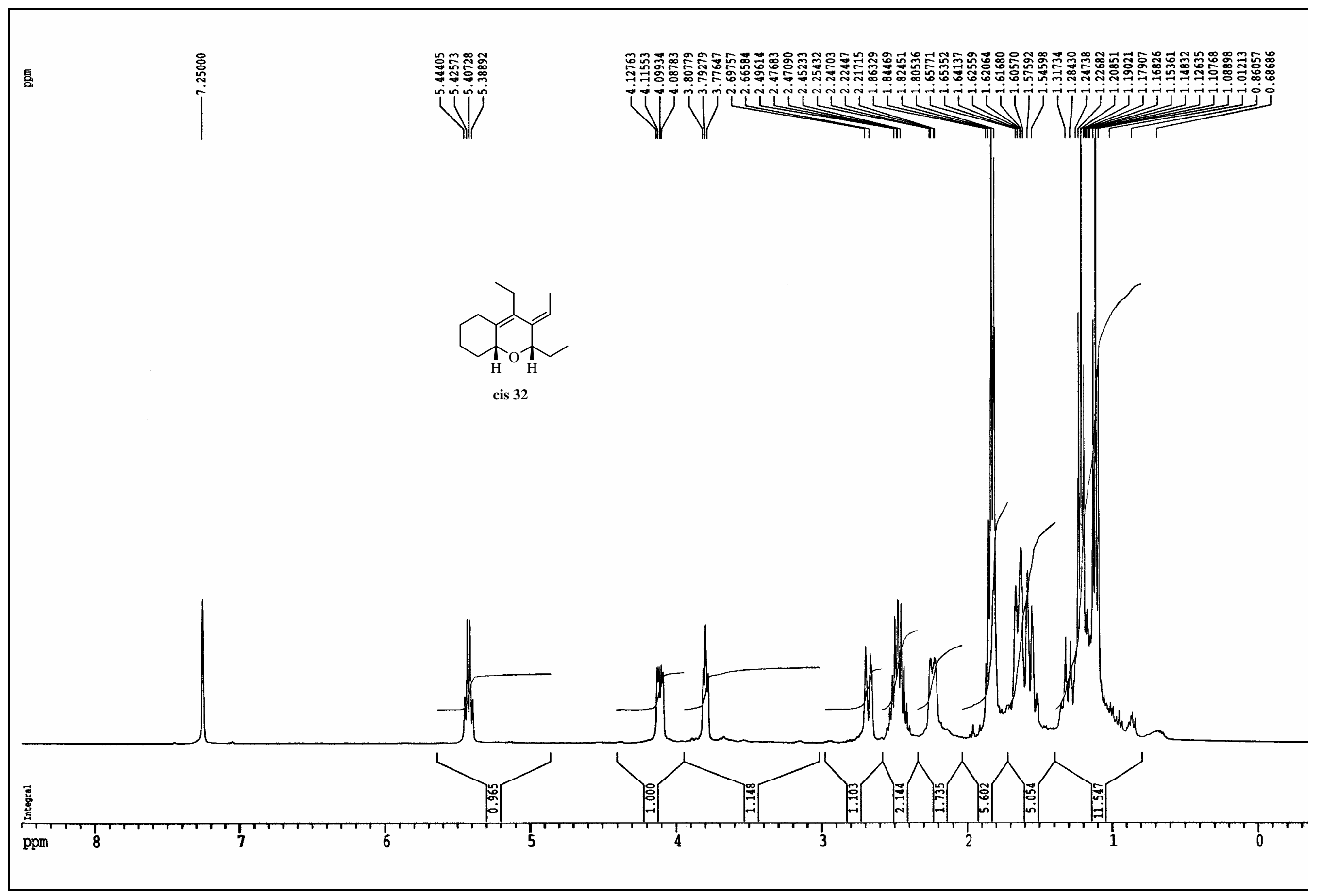




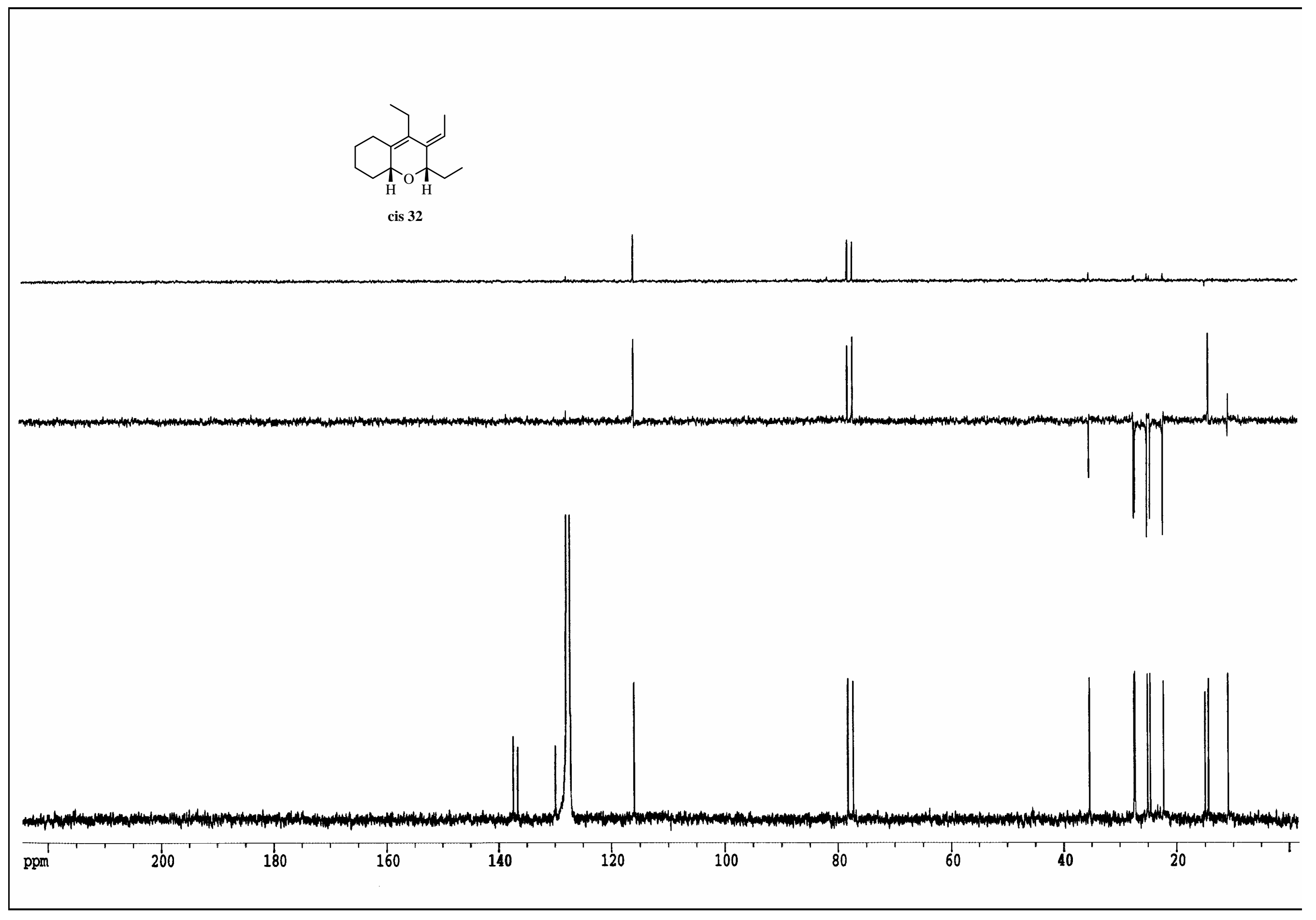




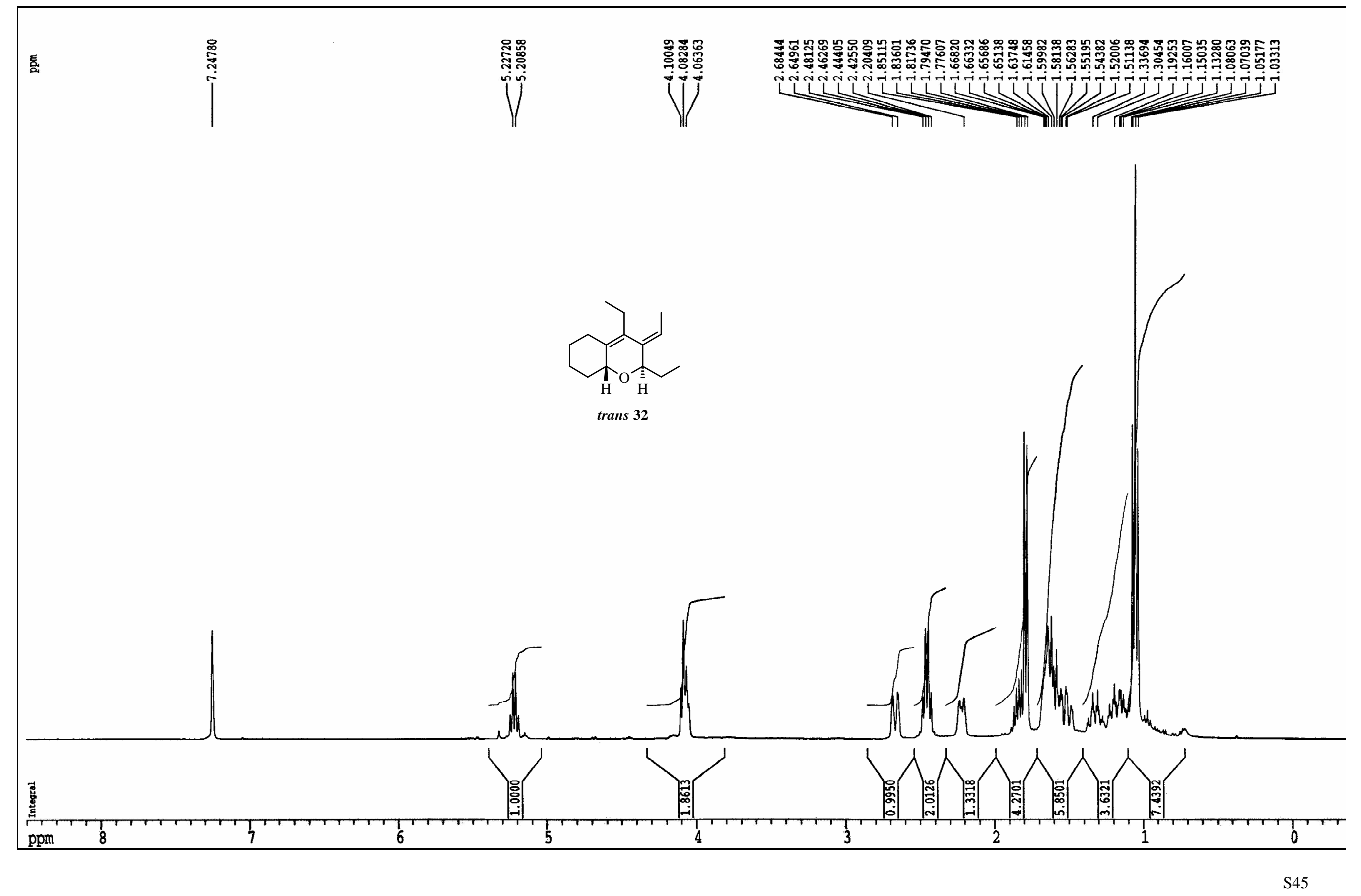




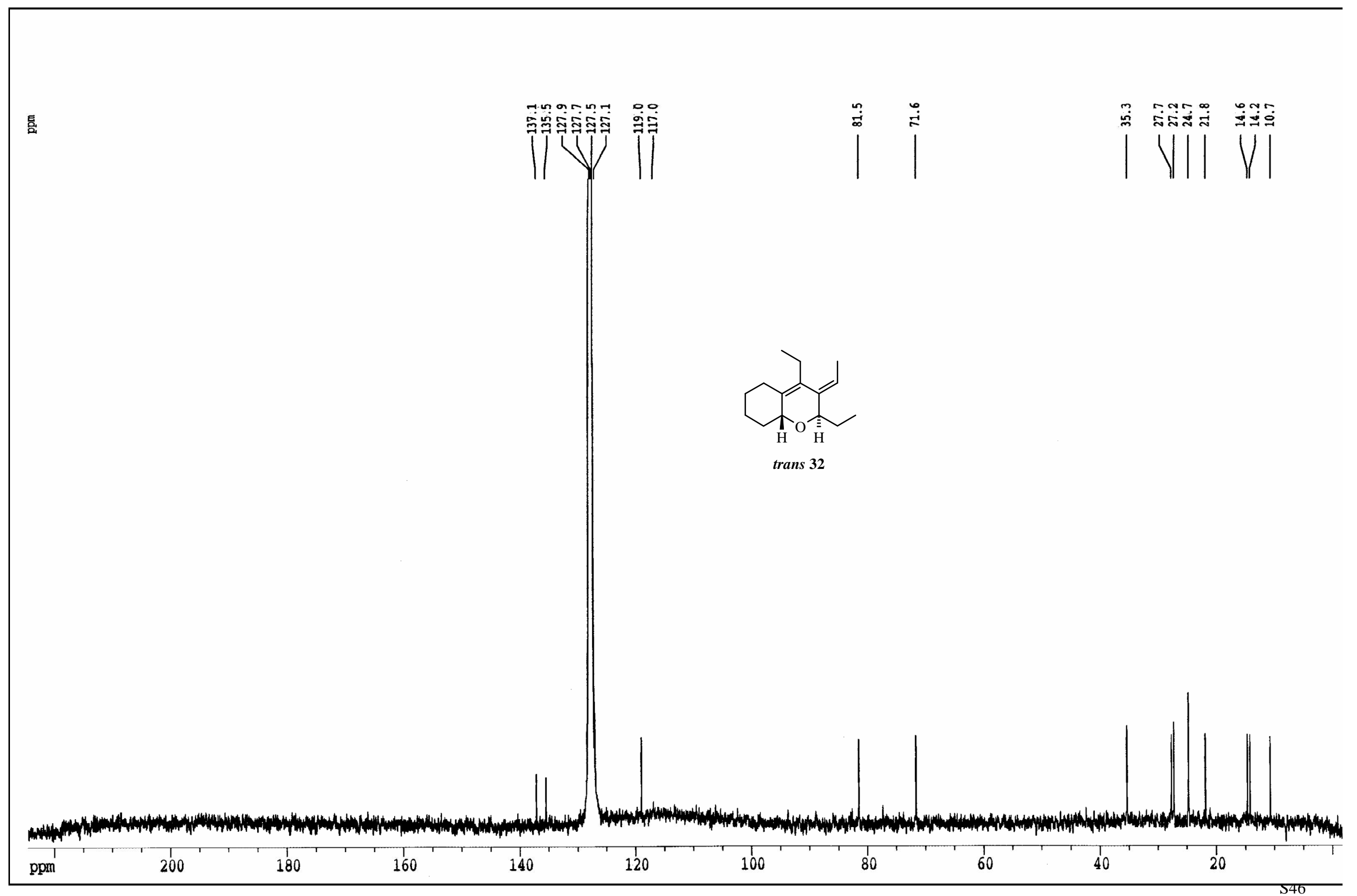




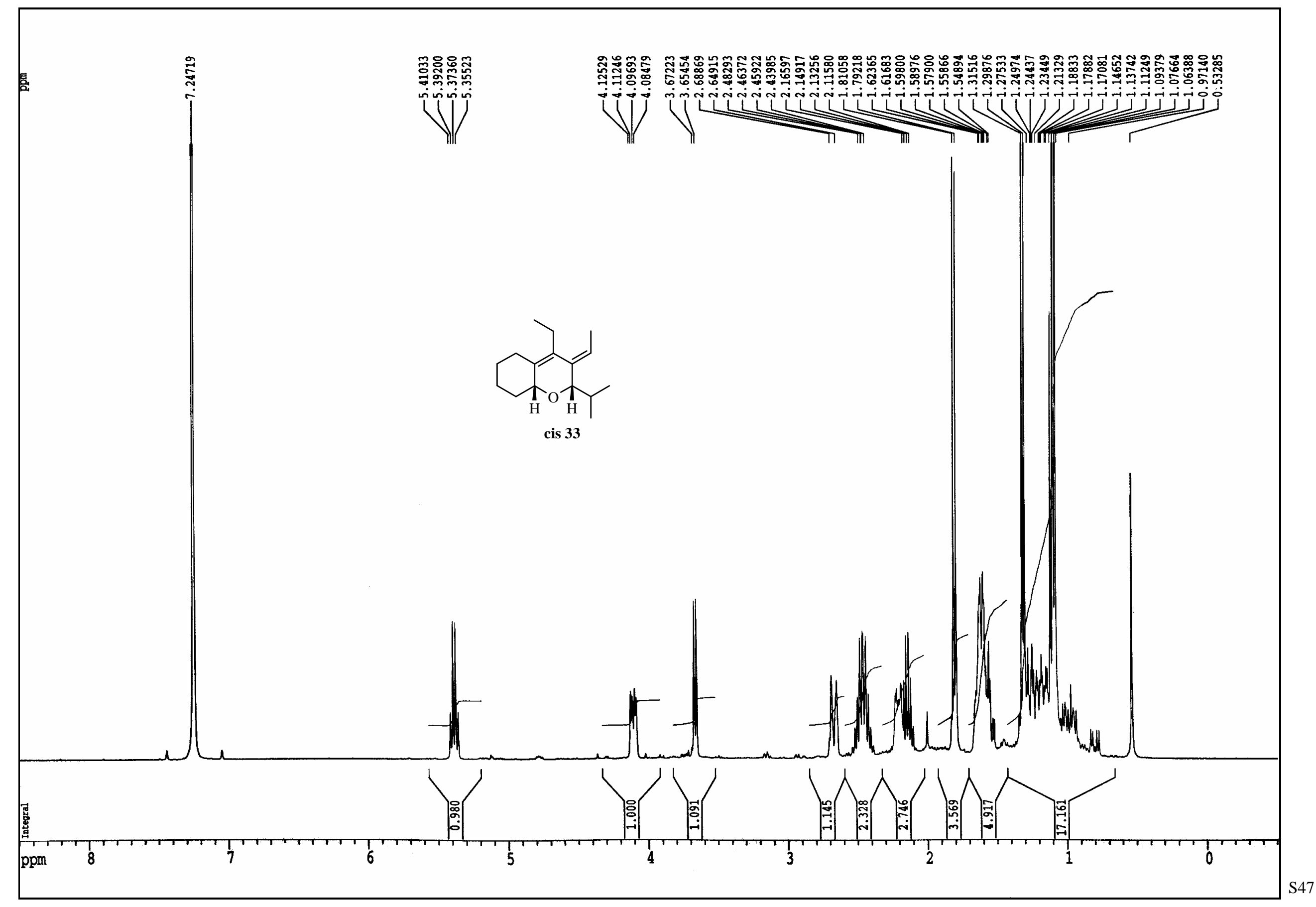




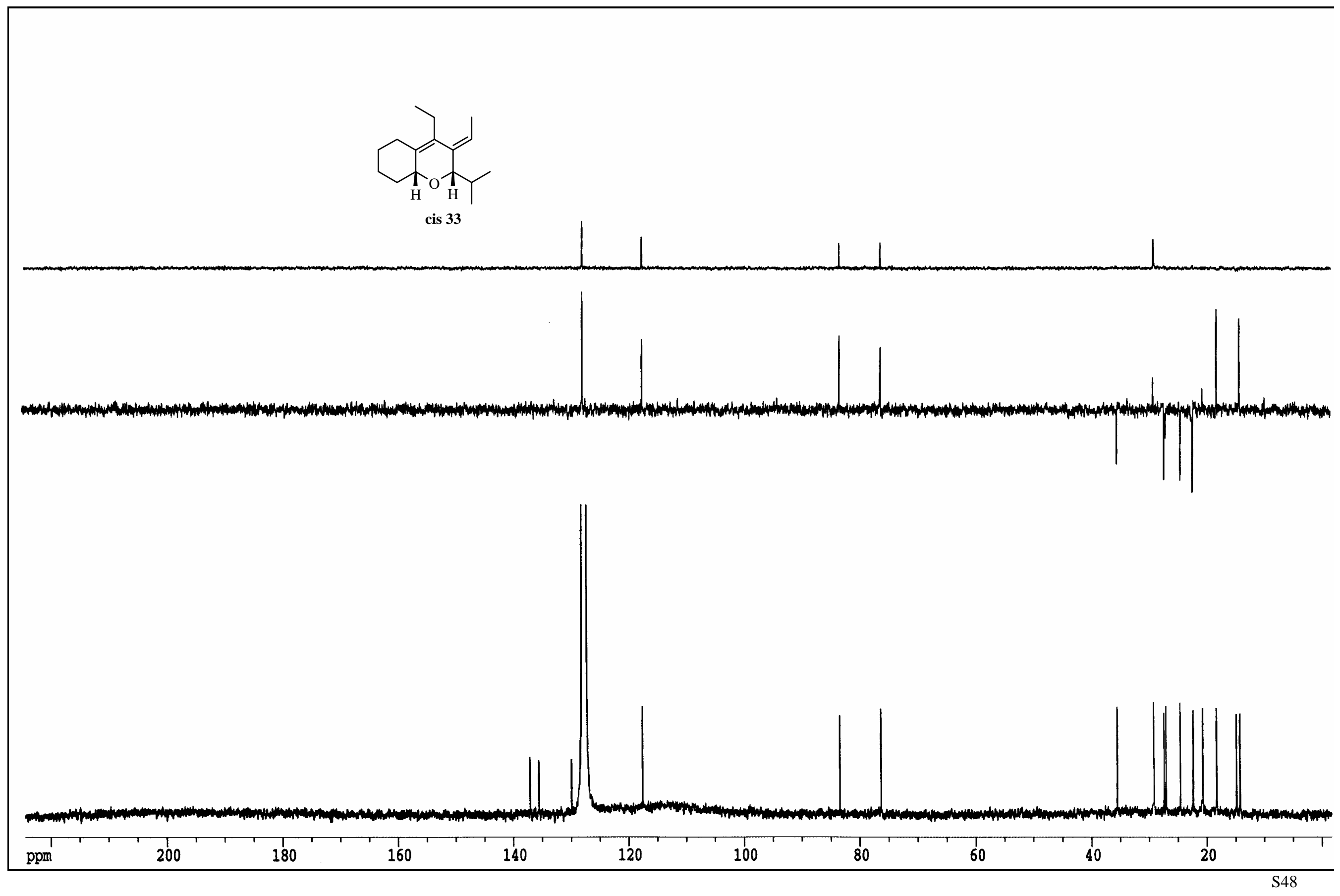




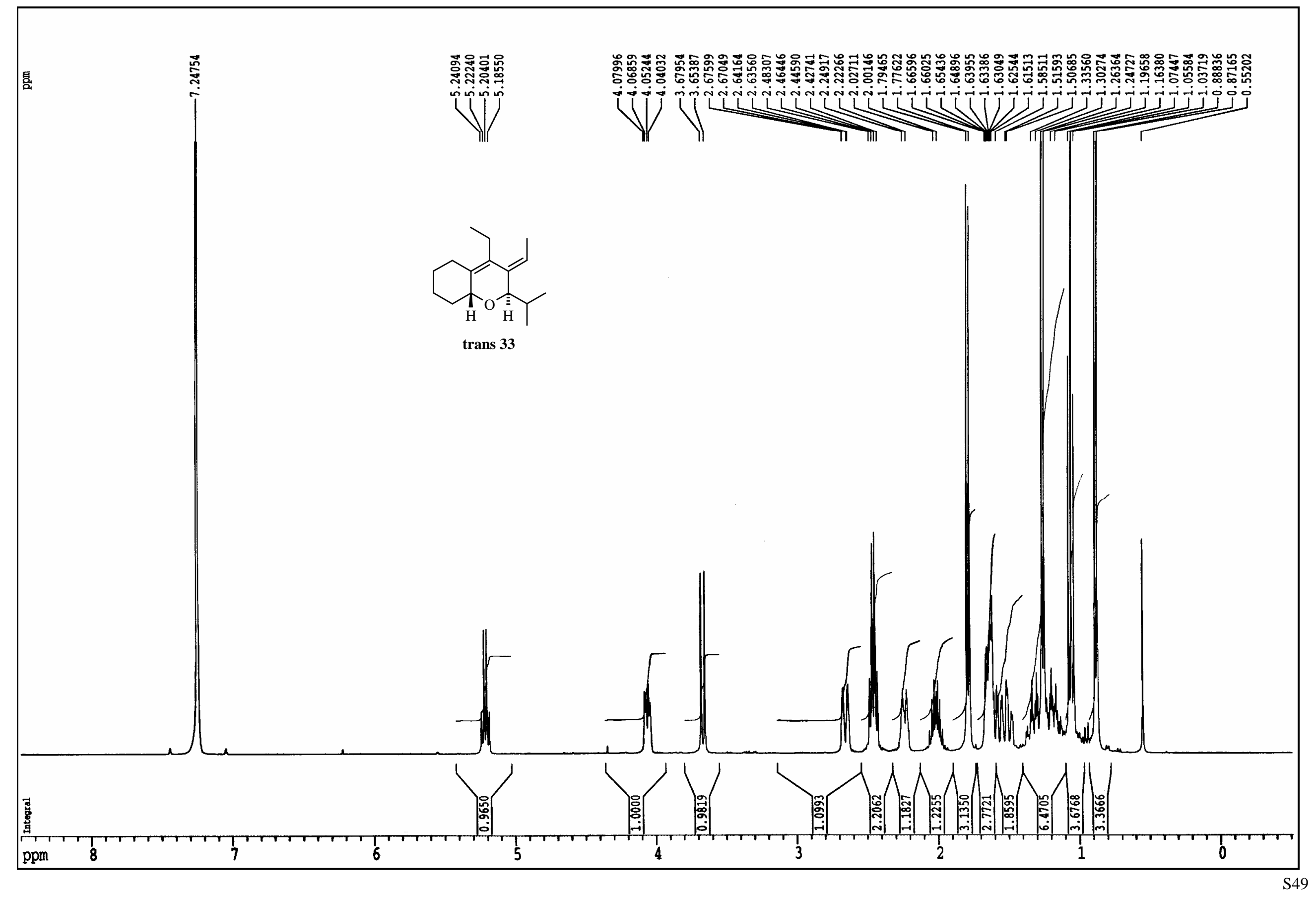




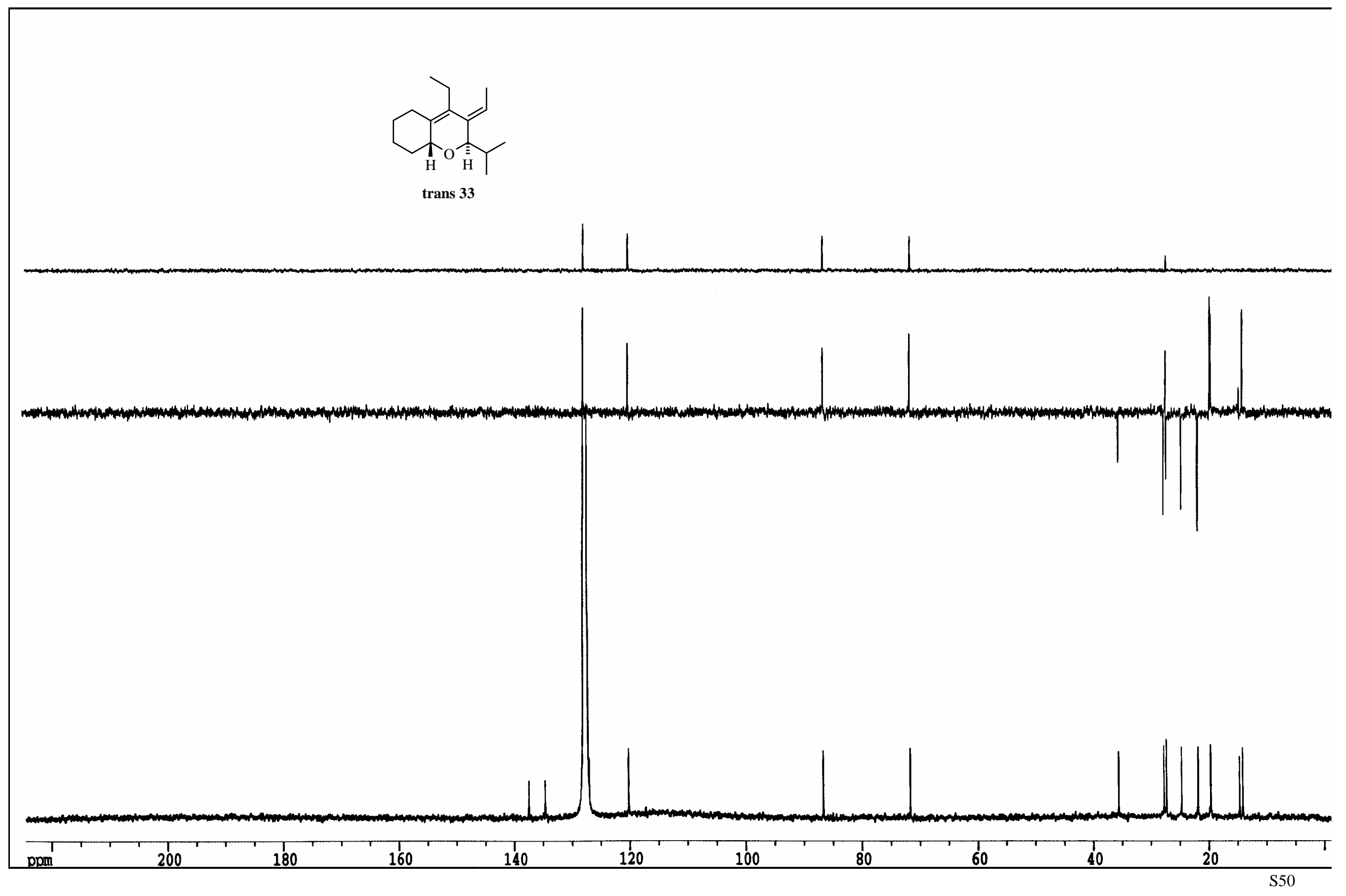




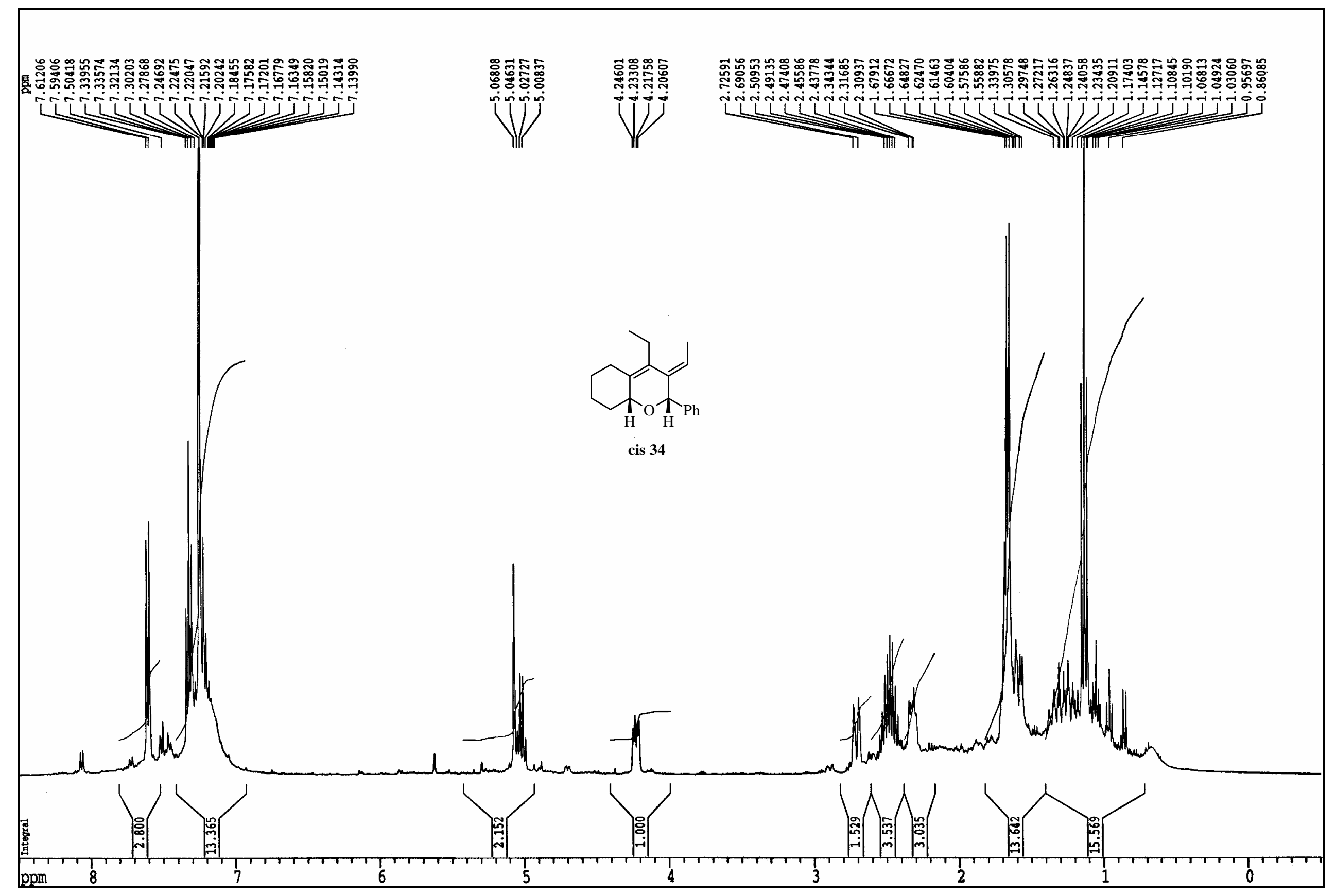




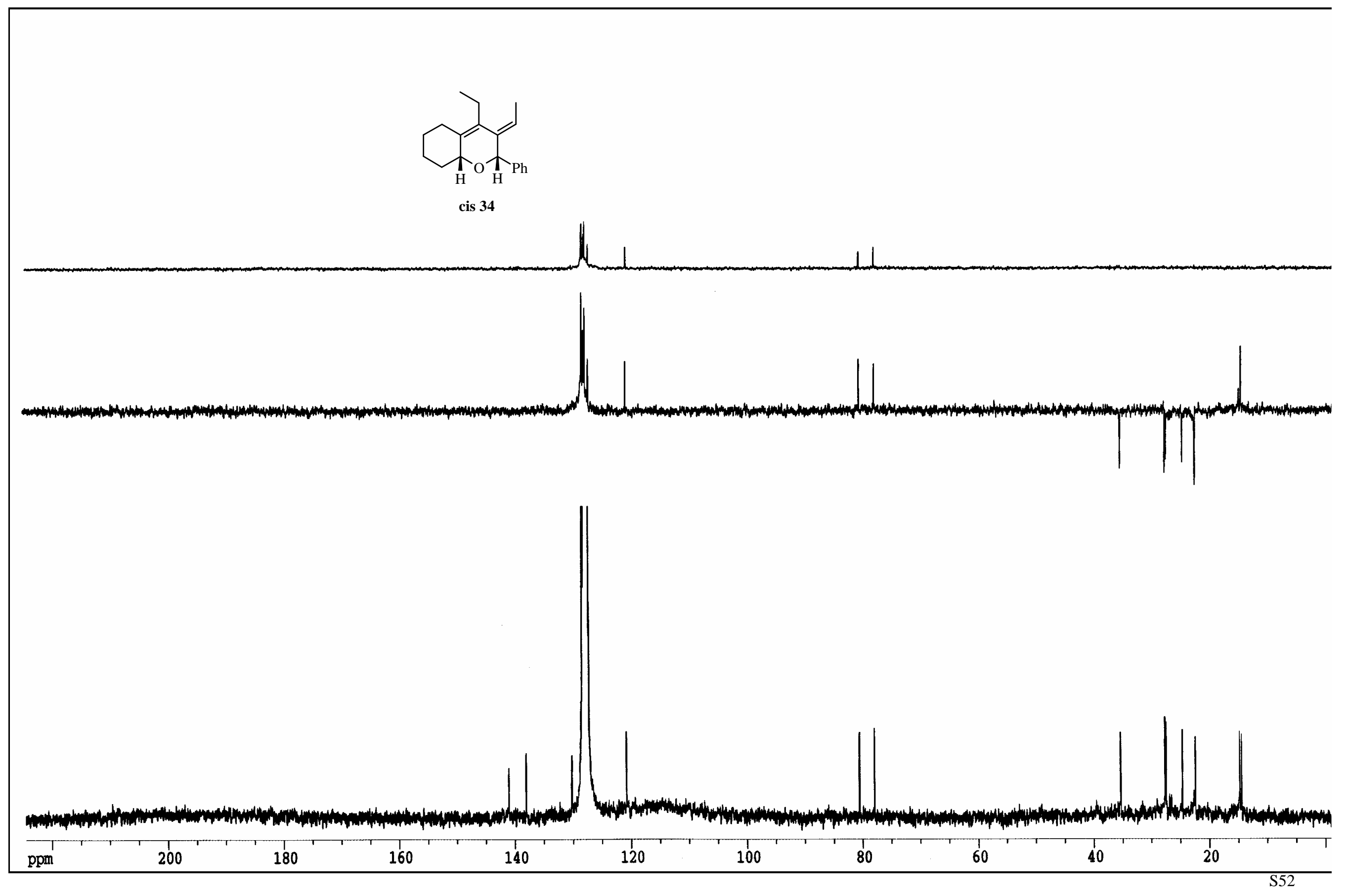




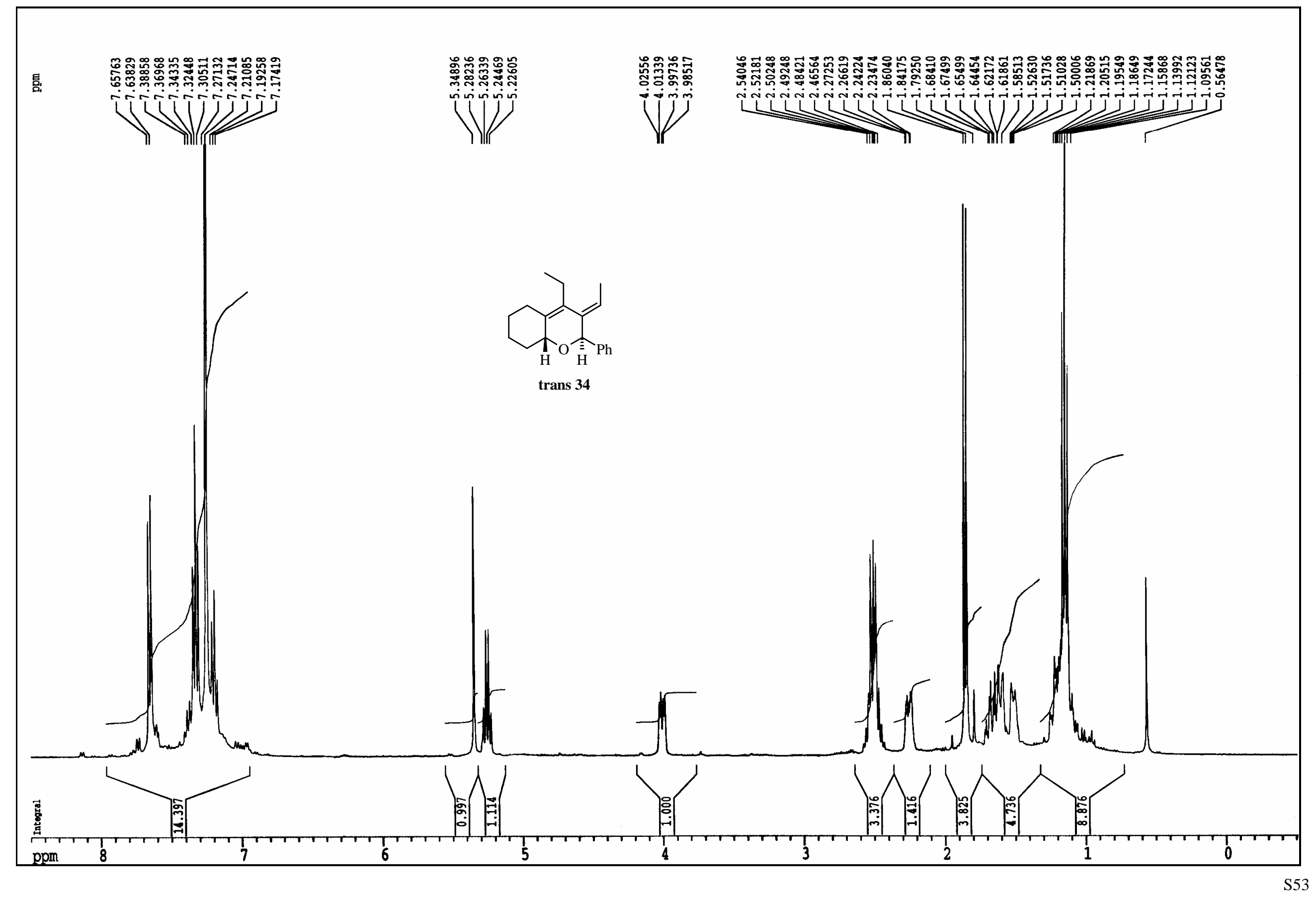




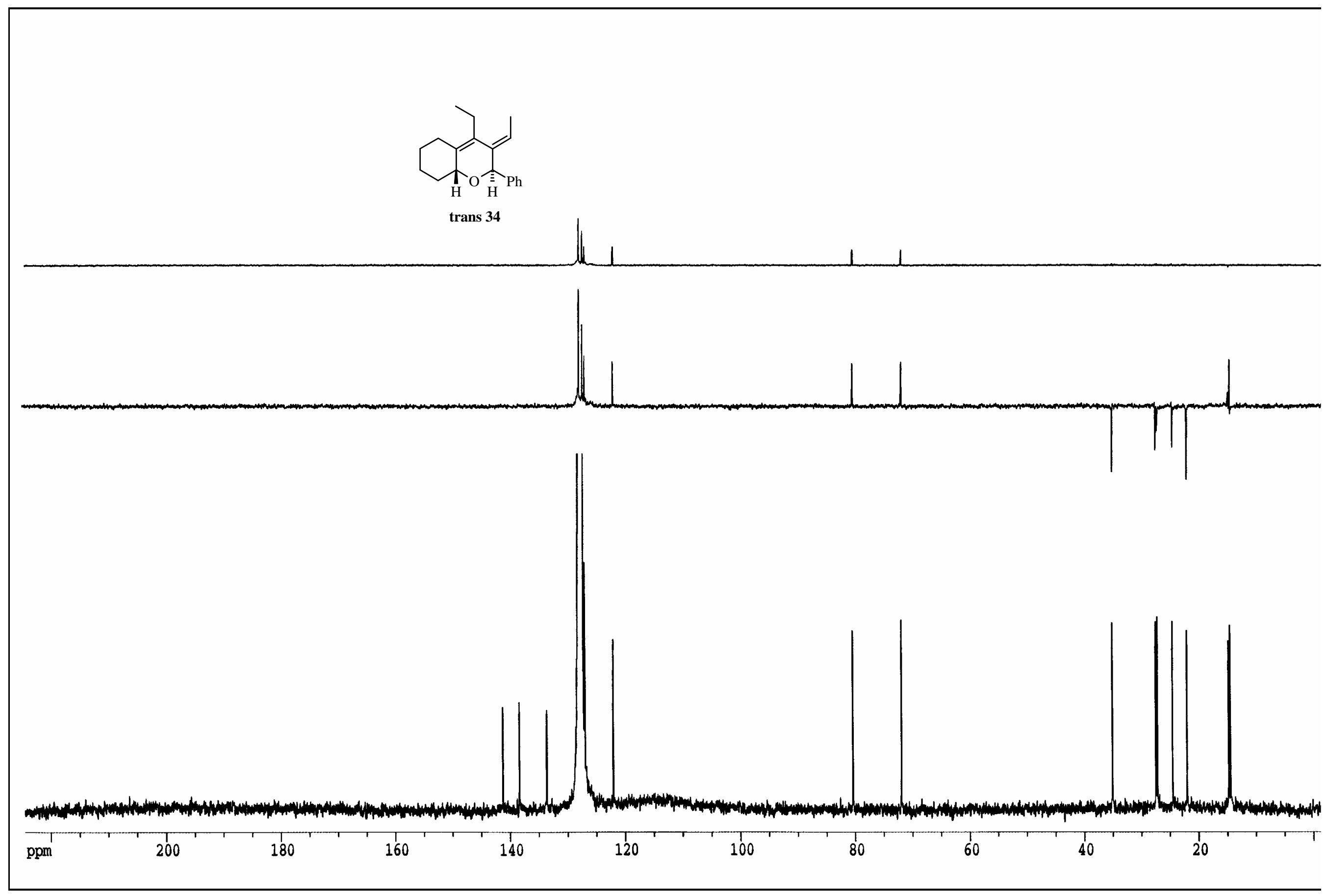




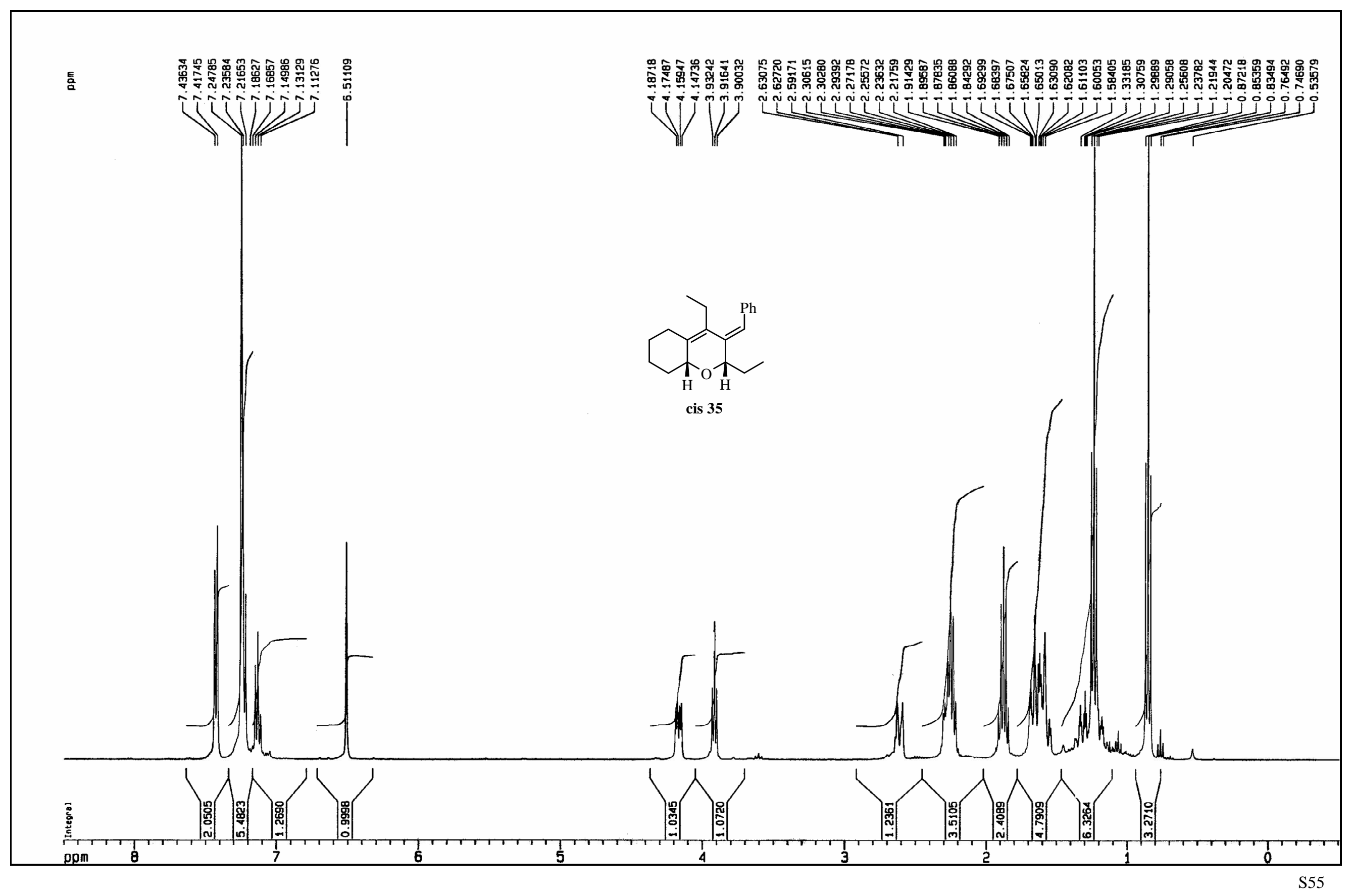




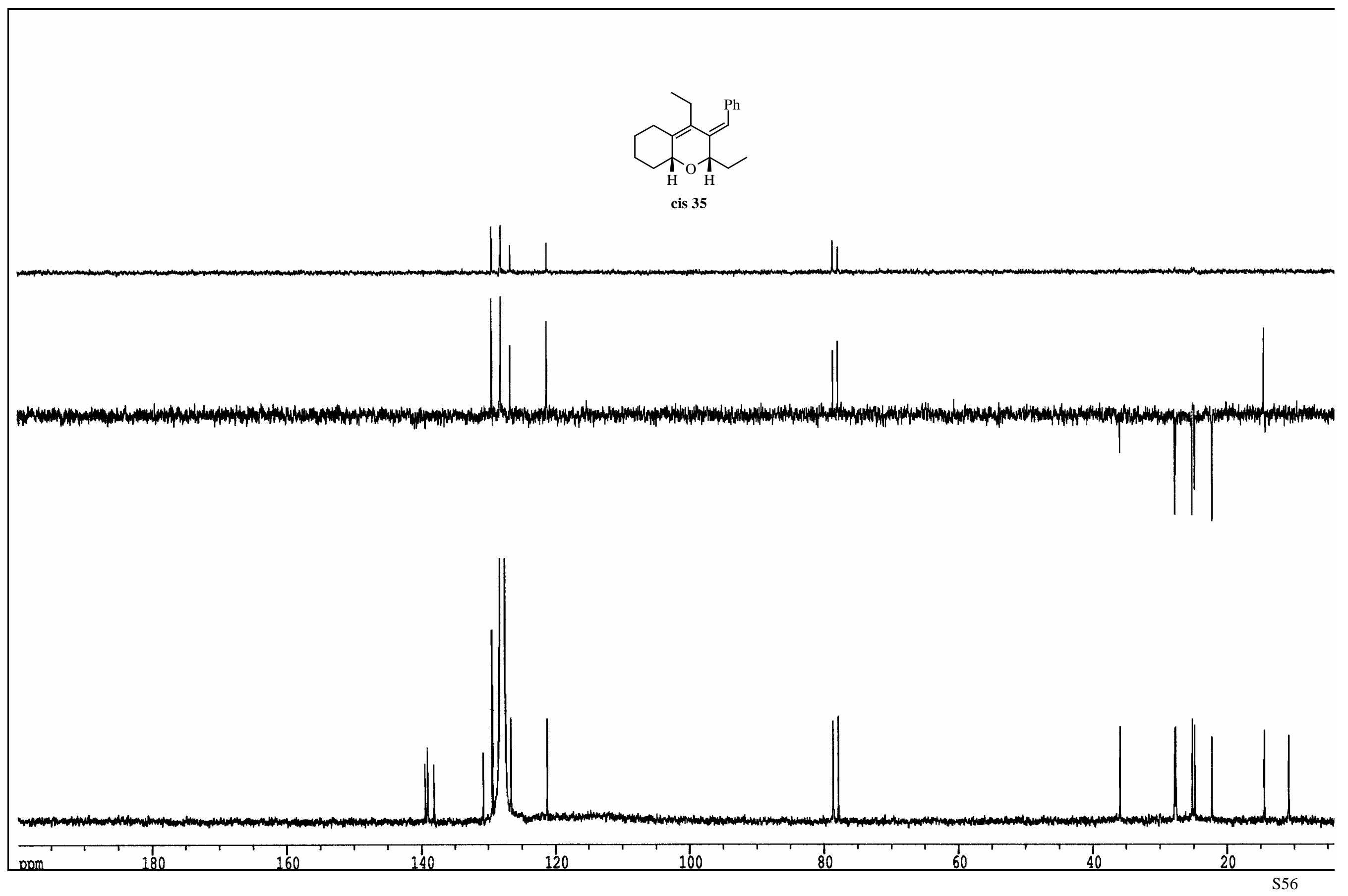




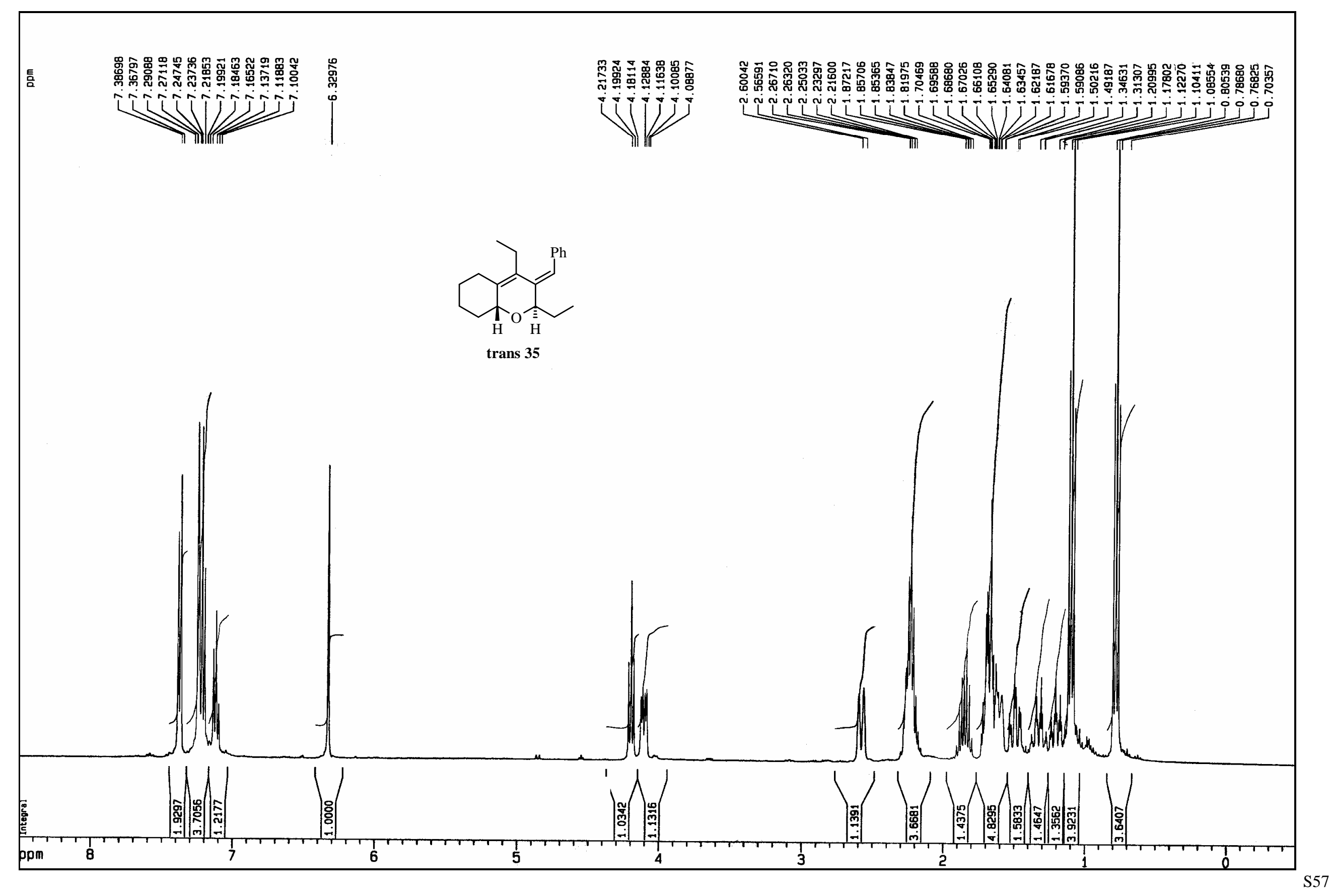




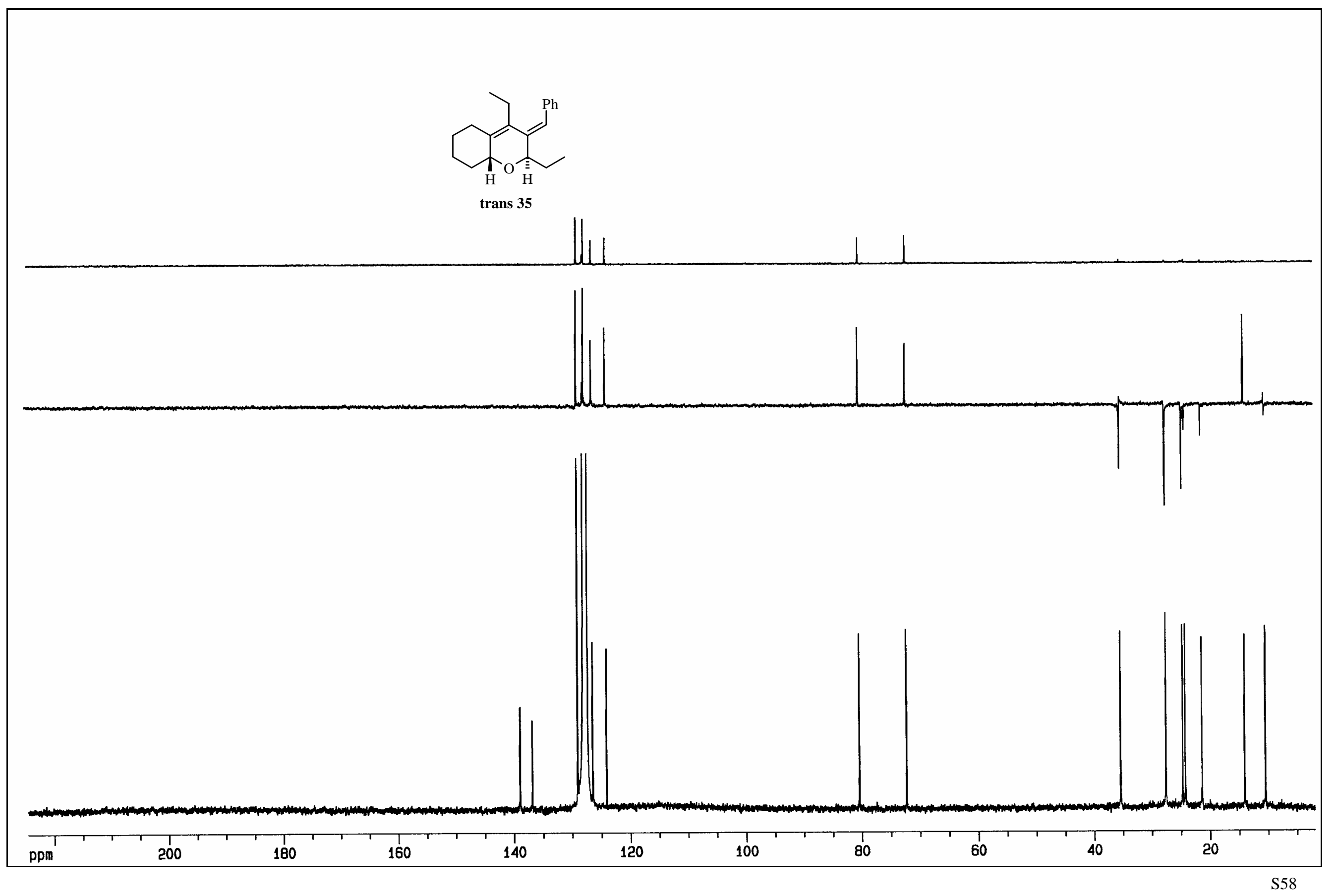




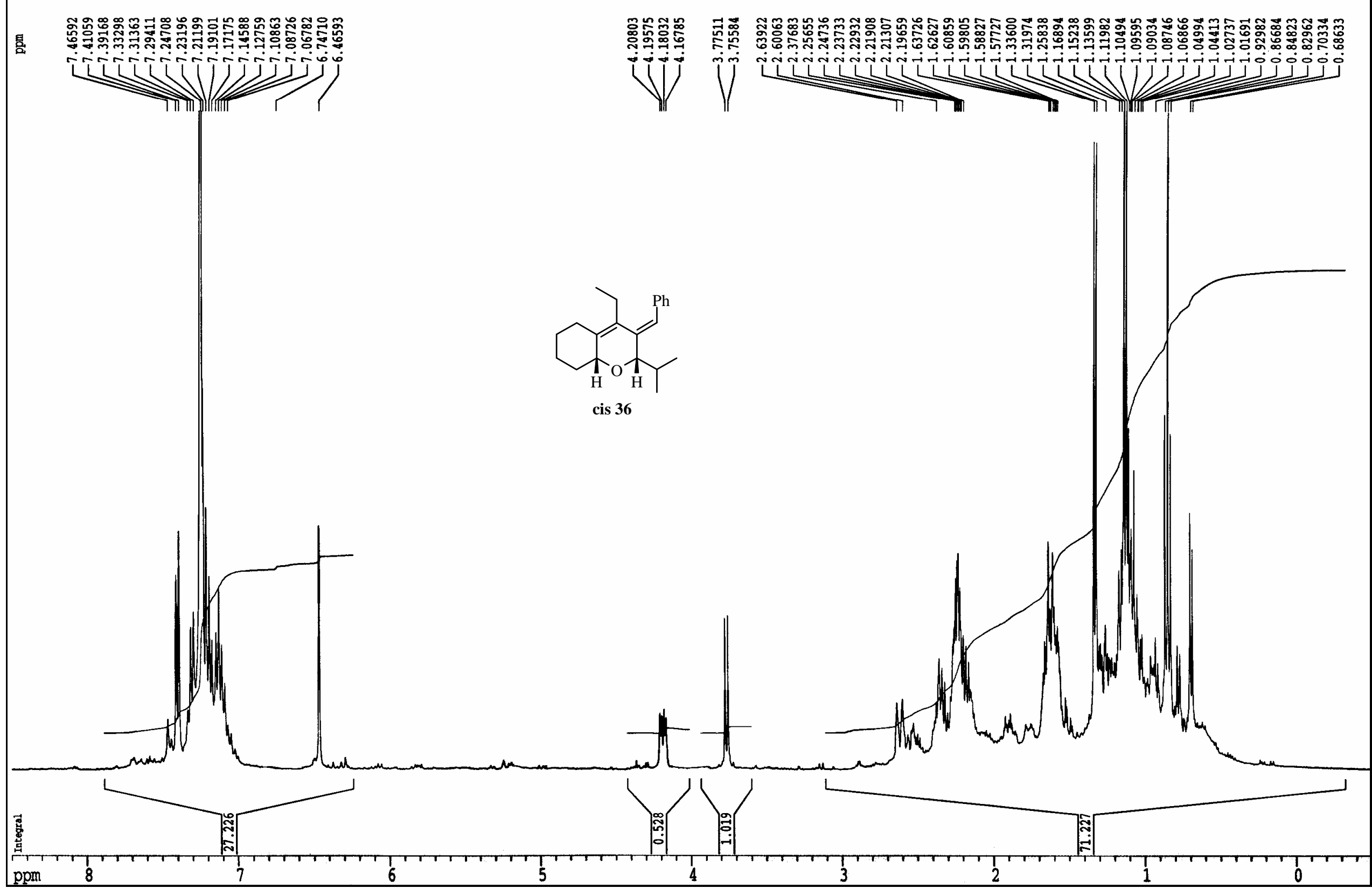




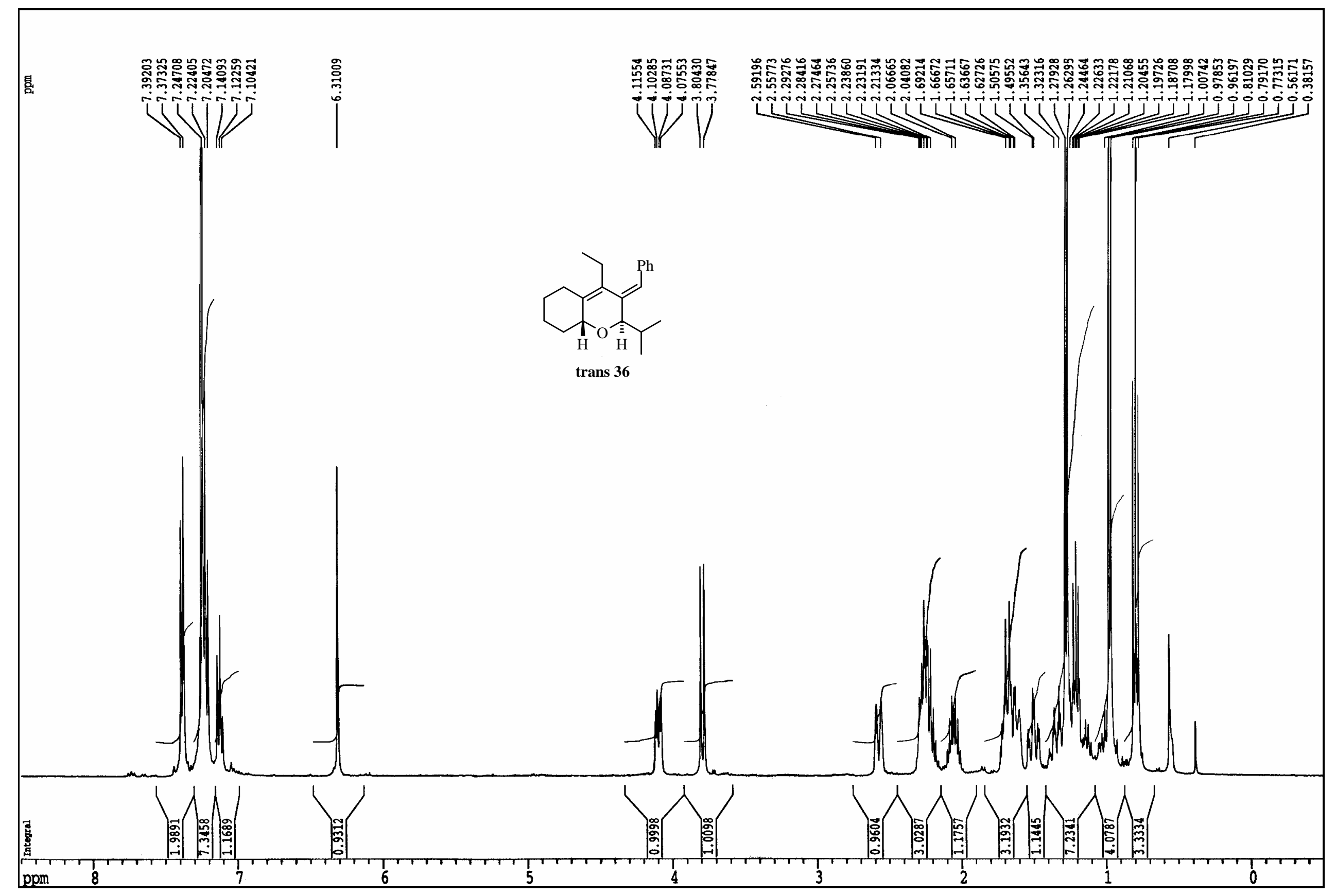




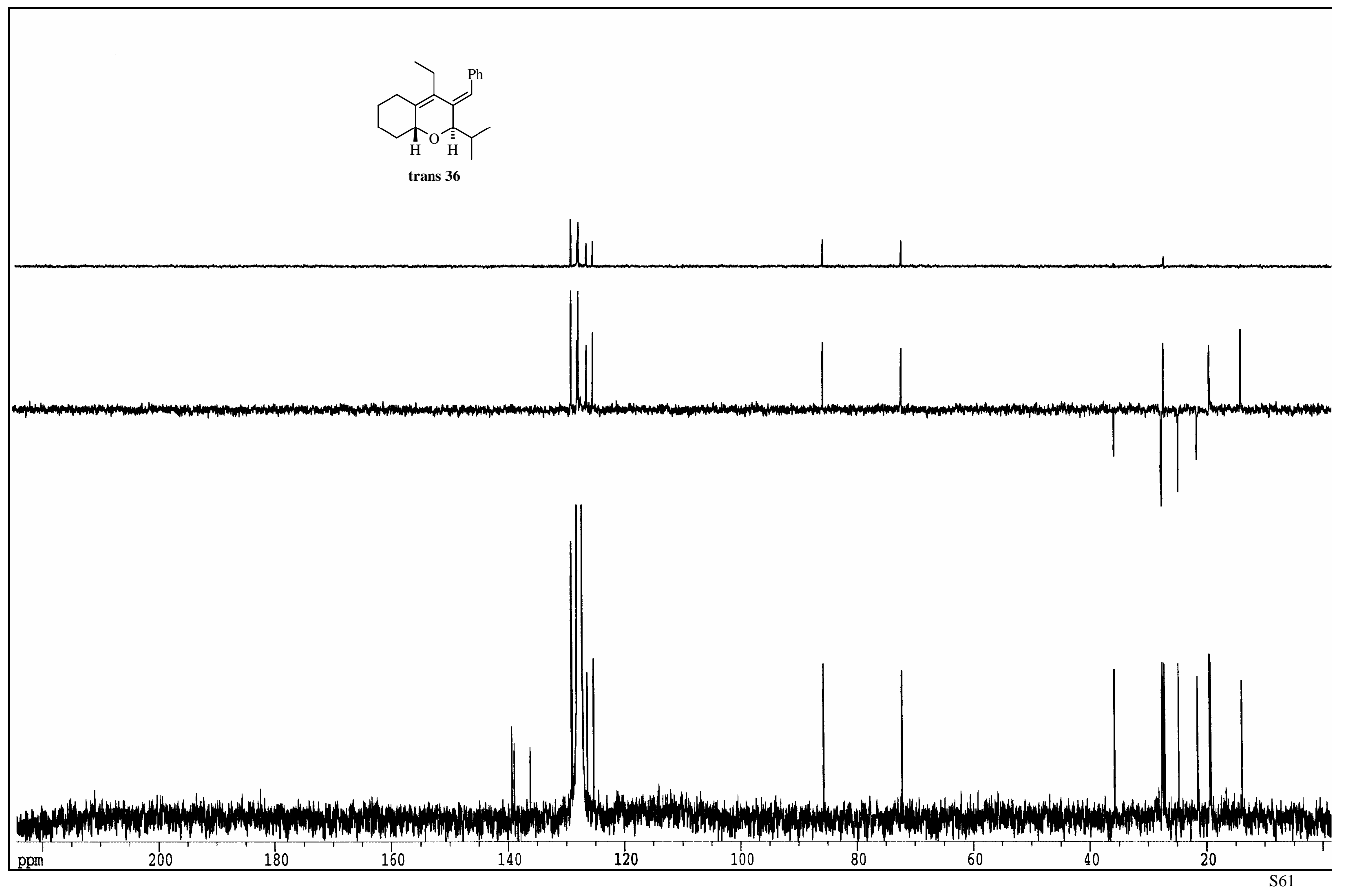




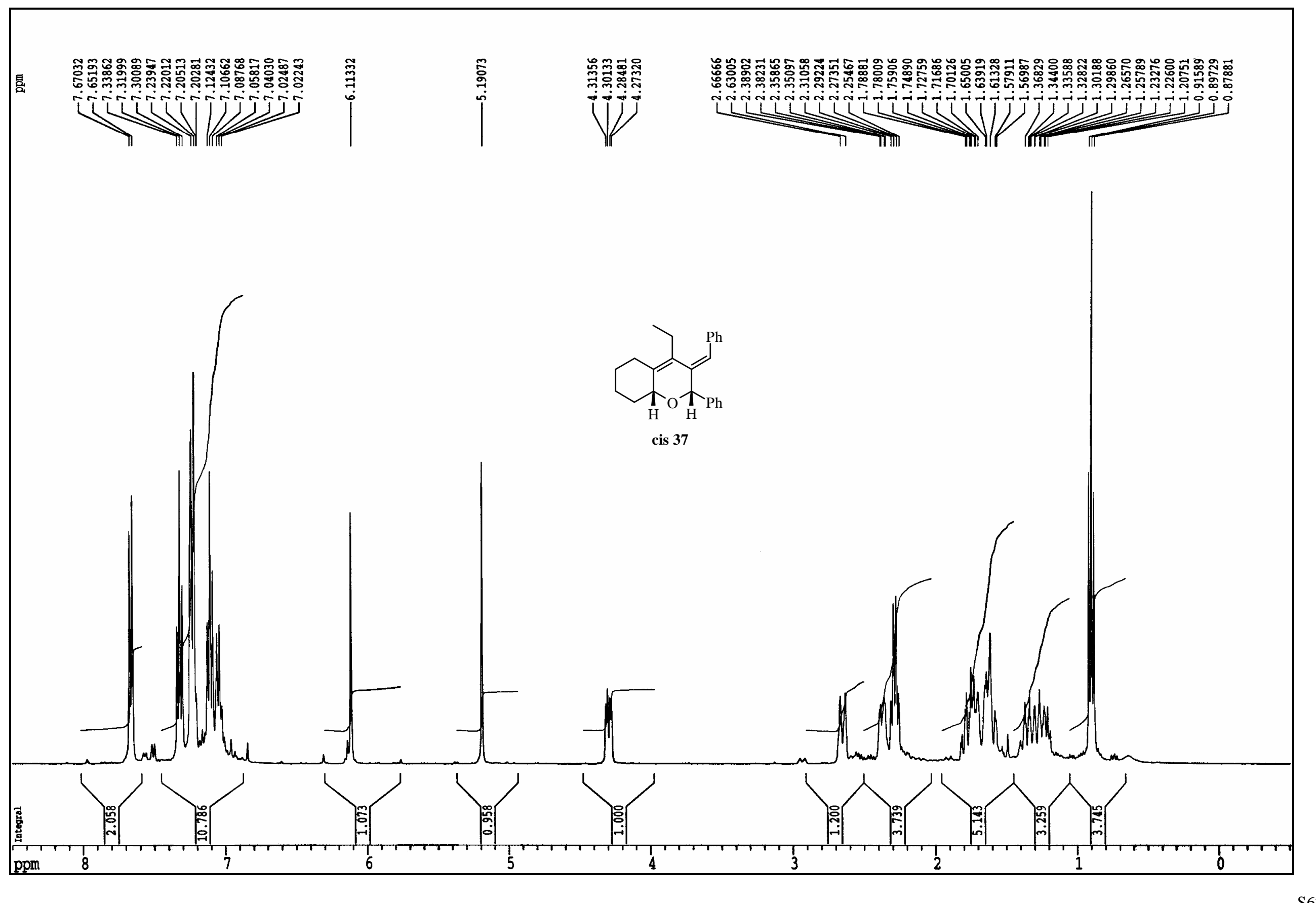




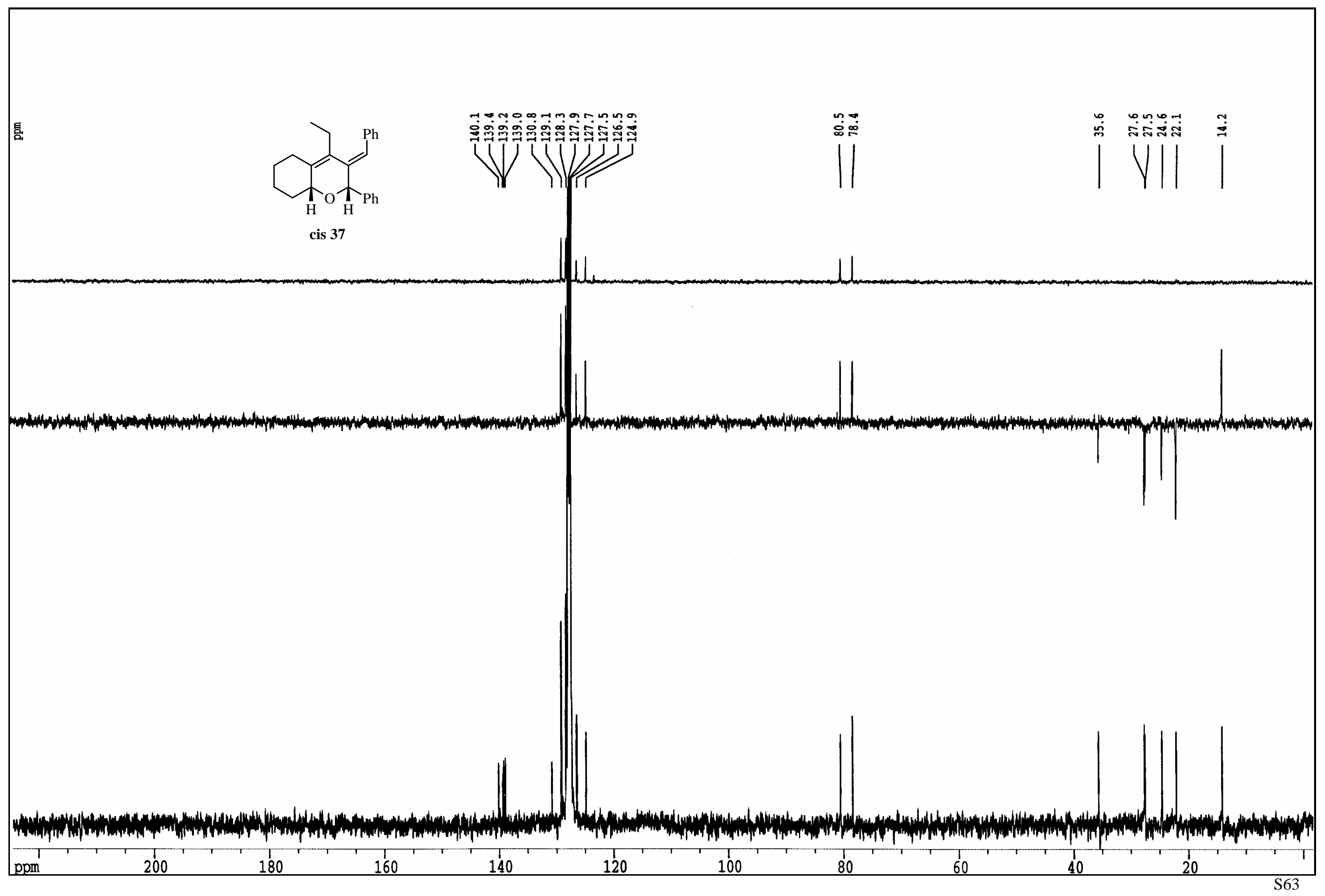




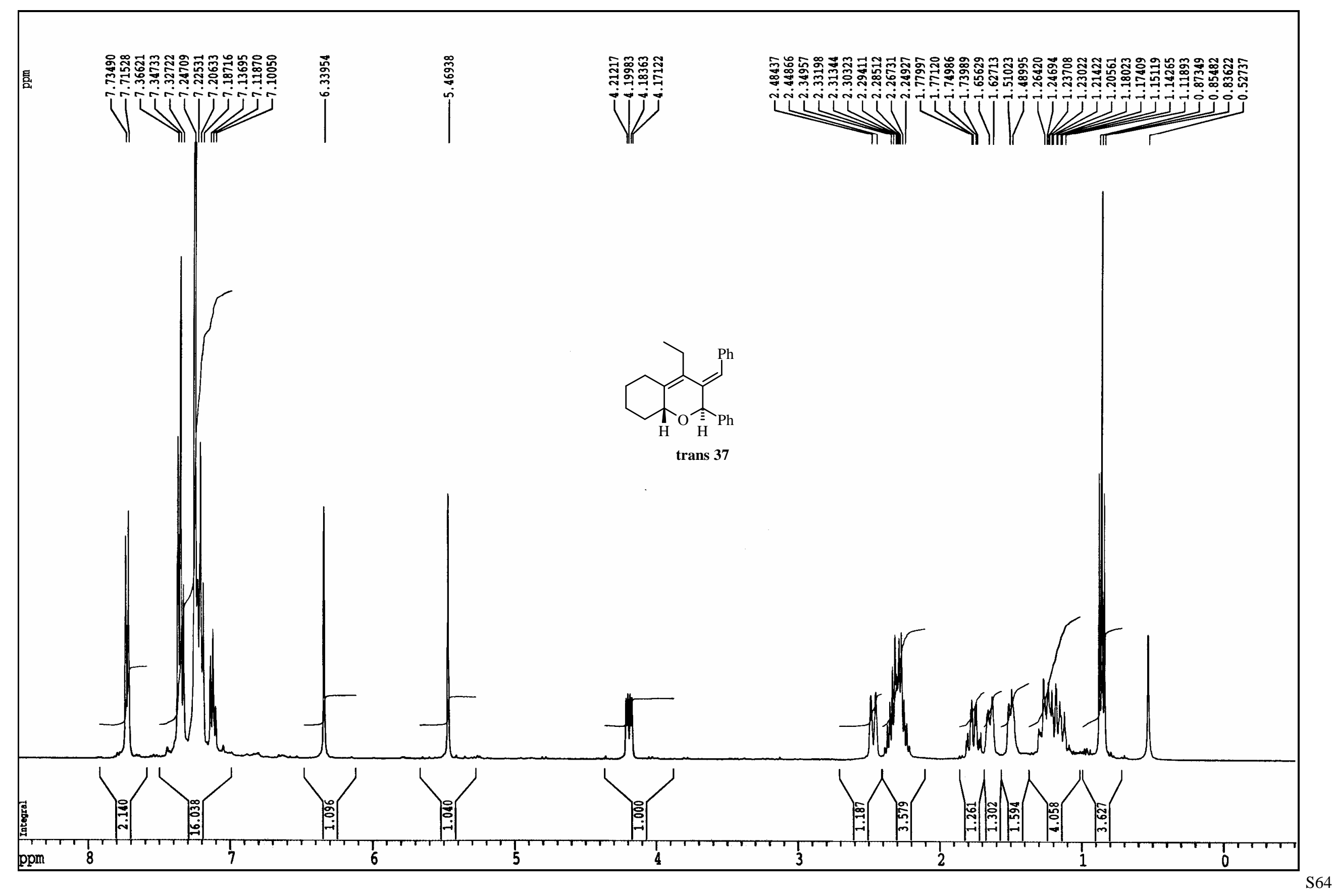




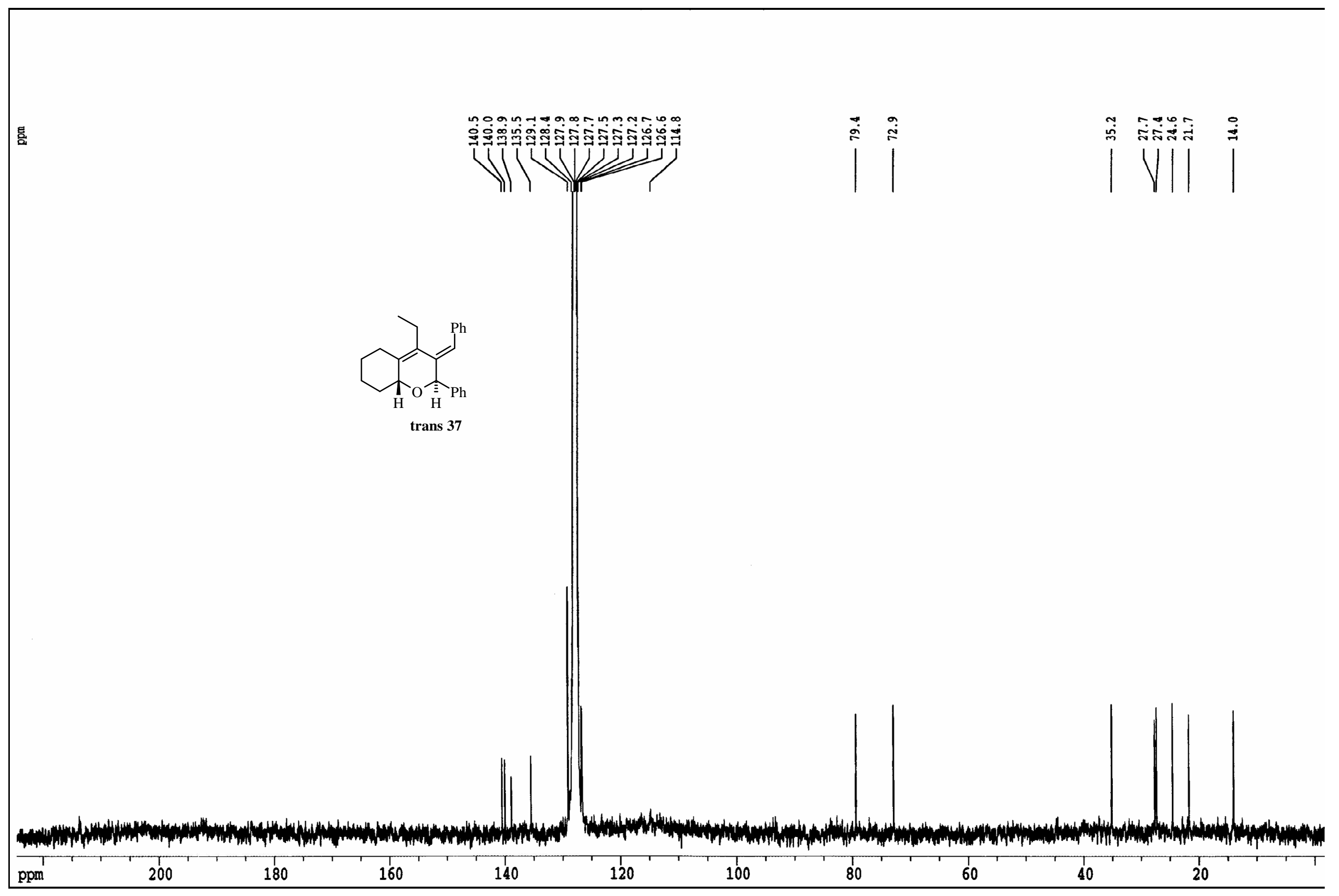




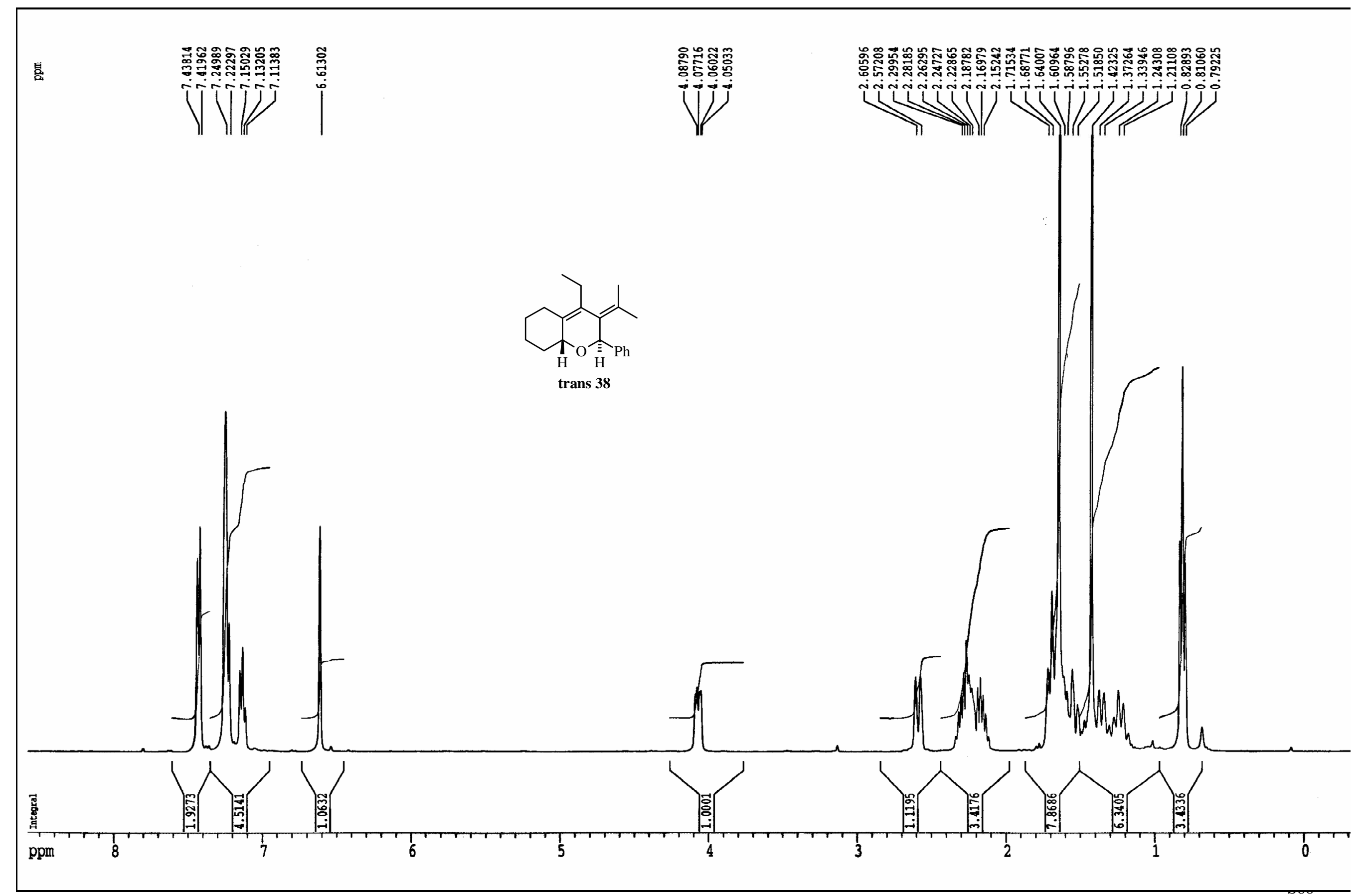




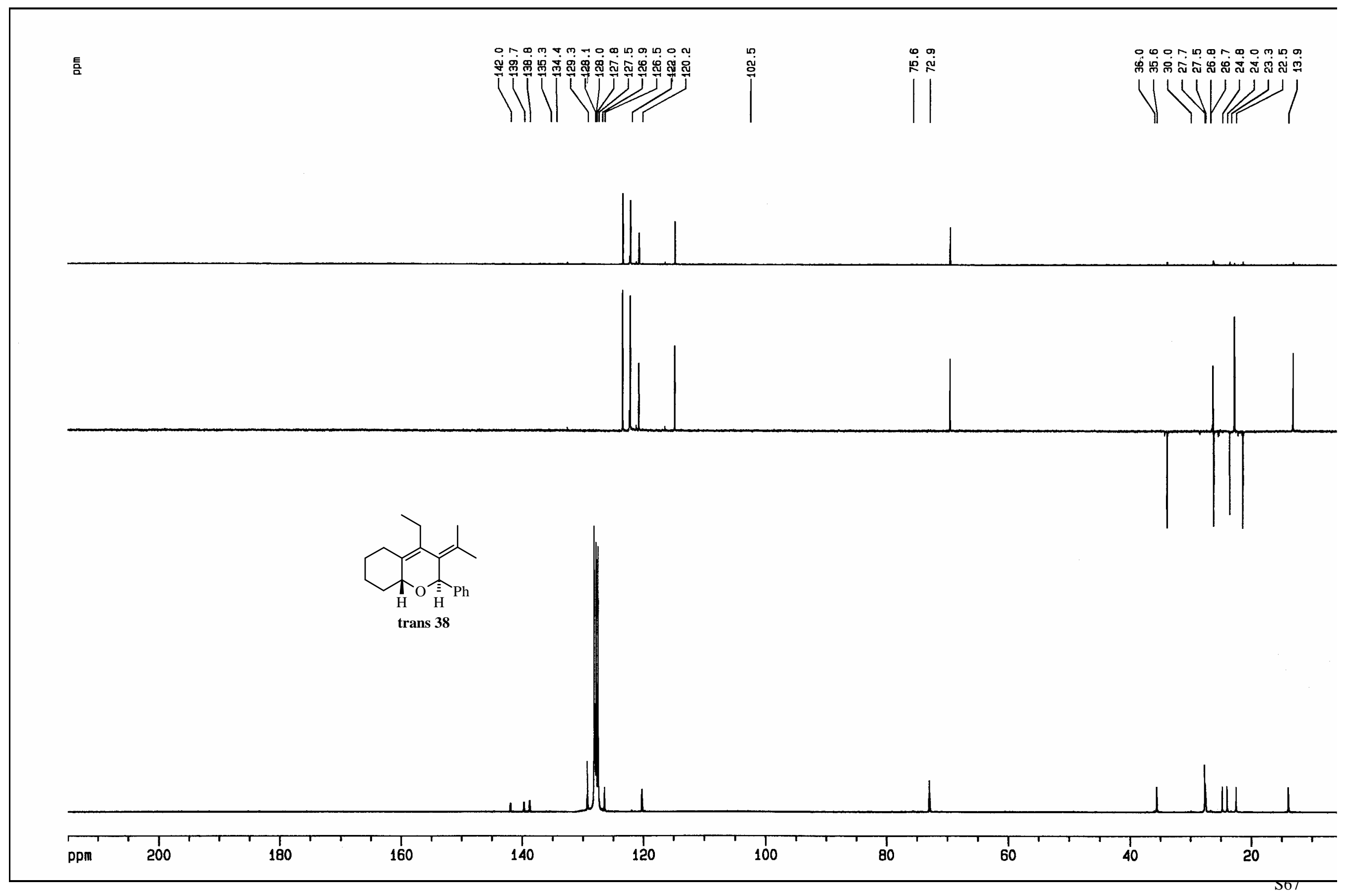




\section{Analysis of the Mulliken charges at the Transition States.}

The analysis of the Mulliken charges at the transition states allow conclusions that are consistent with that obtained from the NBO analysis. The following Table shows the atomic charges of the relevant atoms in the starting materials and transition states for the uncatalyzed reactions. (Mulliken charges calculated at B3LYP/6-31G(d,p)//B3LYP/6-31G(d,p))

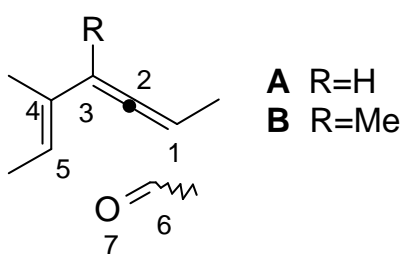

\begin{tabular}{|c|c|c|c|}
\hline Atom & A+ald. & $\mathbf{T S}_{\mathbf{A N}}$ & $\mathbf{T S}_{\mathbf{A X}}$ \\
\hline C1 & -0.280 & -0.121 & -0.111 \\
\hline C2 & 0.280 & 0.019 & -0.009 \\
\hline C3 & -0.291 & 0.143 & -0.139 \\
\hline C4 & 0.159 & 0.142 & 0.162 \\
\hline C5 & -0.122 & -0.010 & -0.027 \\
\hline C6 & 0.298 & 0.221 & 0.219 \\
\hline O7 & -0.383 & 0.485 & -0.469 \\
\hline
\end{tabular}

\begin{tabular}{|c|c|c|}
\hline $\mathbf{B}+$ ald & $\mathbf{T S}_{\mathbf{B N}}$ & $\mathbf{T S}_{\mathbf{B X}}$ \\
\hline-0.284 & -0.116 & -0.109 \\
\hline 0.240 & -0.033 & -0.056 \\
\hline-0.111 & 0.052 & 0.058 \\
\hline 0.126 & 0.120 & 0.128 \\
\hline-0.107 & -0.014 & -0.013 \\
\hline 0.298 & 0.228 & 0.235 \\
\hline-0.383 & -0.492 & -0.490 \\
\hline
\end{tabular}

It can be seen that the biggest change in going from the starting vinyl allenes to the transition states takes place at the central carbon of the allene $(\mathrm{C} 2)$ in all cases, and it occurs at the expense of carbon $\mathrm{C} 1, \mathrm{C} 3$ and $\mathrm{C} 5$. Carbon $\mathrm{C} 4$ suffers very small changes in all cases. The change in $\mathrm{C} 2$ is slightly bigger for vinyl allene $\mathbf{B}$ and for the exo transition states. For the aldehyde, both atoms forming the double bond $(\mathrm{C} 6=\mathrm{O6})$ increase their charges, this effect being more important for the oxygen atom. Adding the charges of all atoms, it can be observed that there is a net charge transfer from the vinyl allene to the aldehyde which is bigger for the transition states involving $\mathbf{B}$ and for the endo cases. $\left(\mathbf{T S}_{\mathrm{AN}}: 0.208 \mathrm{e} ; \mathbf{T S}_{\mathrm{AX}}\right.$ : $0.177 \mathrm{e}$; $\mathbf{T S}_{\mathrm{AN}}$ : 0.220 e; TS $_{\mathbf{B X}}: 0.209$ e). These charge transfers are consistent with a normal demand DielsAlder process.

For the catalyzed processes, the situation is similar but with larger differences between the starting compounds and the transition states. In these cases, the charge transferred to the aldehyde-BF 3 complex is also larger $\left(\mathbf{c T S}_{\mathbf{A N}}: 0.320\right.$ e; $\mathbf{c T S}_{\mathbf{A X}}: 0.325$ e; $\mathbf{c T S}_{\mathbf{B N}}: 0.300$ e; $\mathbf{c T S}_{\mathbf{B X}}$ : $0.299 \mathrm{e}$ ) and is consistent with a more polar transition state as found using NBO analysis.

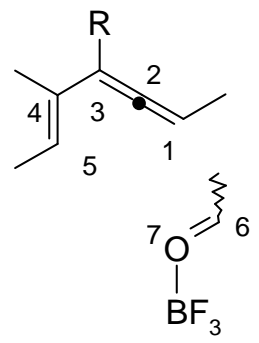

\begin{tabular}{|c|c|c|c|}
\hline Atom & $\mathbf{A}+$ ald. $+\mathrm{BF}_{3}$ & $\mathbf{c T S}_{\mathbf{A N}}$ & $\mathbf{c T S}_{\mathbf{A X}}$ \\
\hline $\mathrm{C} 1$ & -0.280 & -0.100 & -0.110 \\
\hline C2 & 0.280 & 0.013 & -0.019 \\
\hline C3 & -0.291 & -0.124 & -0.086 \\
\hline C4 & 0.159 & 0.139 & 0.143 \\
\hline C5 & -0.122 & -0.055 & -0.039 \\
\hline C6 & 0.311 & 0.202 & 0.202 \\
\hline O7 & -0.373 & -0.434 & -0.452 \\
\hline B & 0.680 & 0.710 & 0.723 \\
\hline F & -0.276 & -0.323 & -0.330 \\
\hline
\end{tabular}

\begin{tabular}{|c|c|c|}
\hline $\mathbf{B}+$ ald $+\mathrm{BF}_{3}$ & $\mathbf{c T S}_{\mathbf{B N}}$ & $\mathbf{c T S}_{\mathbf{B X}}$ \\
\hline-0.283 & -0.101 & -0.18 \\
\hline 0.239 & -0.049 & -0.063 \\
\hline-0.111 & 0.065 & 0.092 \\
\hline 0.126 & 0.126 & 0.134 \\
\hline-0.106 & -0.066 & -0.056 \\
\hline 0.311 & 0.216 & 0.214 \\
\hline-0.373 & -0.430 & -0.439 \\
\hline 0.680 & 0.708 & 0.709 \\
\hline-0.276 & -0.314 & -0.310 \\
\hline
\end{tabular}

(Only one $\mathrm{F}$ atom is included in the table, the other two have similar values) 


\section{A}

$\mathrm{E}(\mathrm{RB}+\mathrm{HF}-\mathrm{LYP})=-312.026677104$ A.U. Zero-point correction $=0.173983$ (Hartree/Particle)

\begin{tabular}{|c|c|c|c|c|c|}
\hline \multirow{2}{*}{$\begin{array}{l}\text { Center } \\
\text { Number }\end{array}$} & \multirow{2}{*}{$\begin{array}{l}\text { Atomic } \\
\text { Number }\end{array}$} & \multirow{2}{*}{$\begin{array}{c}\text { Atomic } \\
\text { Type }\end{array}$} & \multicolumn{3}{|c|}{ Coordinates (Angstroms) } \\
\hline & & & $\mathrm{X}$ & $Y$ & Z \\
\hline 1 & 6 & 0 & -0.222121 & -1.060984 & -0.316176 \\
\hline 2 & 6 & 0 & -1.370976 & -0.426780 & -0.408796 \\
\hline 3 & 6 & 0 & 1.112234 & -0.463910 & -0.049876 \\
\hline 4 & 6 & 0 & -2.517312 & 0.197596 & -0.497111 \\
\hline 5 & 6 & 0 & -3.463877 & 0.449177 & 0.653504 \\
\hline 6 & 6 & 0 & 1.325676 & 0.858559 & -0.179500 \\
\hline 7 & 6 & 0 & 2.589684 & 1.626614 & 0.068645 \\
\hline 8 & 6 & 0 & 2.161990 & -1.470663 & 0.359477 \\
\hline 9 & 1 & 0 & -0.237620 & -2.146205 & -0.435865 \\
\hline 10 & 1 & 0 & -2.832515 & 0.569720 & -1.474643 \\
\hline 11 & 1 & 0 & -4.439081 & -0.015504 & 0.463877 \\
\hline 12 & 1 & 0 & -3.066854 & 0.048173 & 1.588677 \\
\hline 13 & 1 & 0 & -3.640751 & 1.523408 & 0.785024 \\
\hline 14 & 1 & 0 & 0.477107 & 1.462005 & -0.501314 \\
\hline 15 & 1 & 0 & 2.903658 & 2.165556 & -0.834969 \\
\hline 16 & 1 & 0 & 3.424070 & 0.998383 & 0.387684 \\
\hline 17 & 1 & 0 & 2.434682 & 2.389909 & 0.842859 \\
\hline 18 & 1 & 0 & 1.878880 & -1.972325 & 1.293392 \\
\hline 19 & 1 & 0 & 3.146611 & -1.024657 & 0.504976 \\
\hline 20 & 1 & 0 & 2.260021 & -2.256129 & -0.400694 \\
\hline
\end{tabular}

\section{B}

$\mathrm{E}(\mathrm{RB}+\mathrm{HF}-\mathrm{LYP})=-351.344194968 \quad \mathrm{~A} \cdot \mathrm{U}$.

Zero-point correction $=0.202226$ (Hartree/Particle)

\begin{tabular}{|c|c|c|c|c|c|}
\hline \multirow{2}{*}{$\begin{array}{l}\text { Center } \\
\text { Number }\end{array}$} & \multirow{2}{*}{$\begin{array}{l}\text { Atomic } \\
\text { Number }\end{array}$} & \multirow{2}{*}{$\begin{array}{c}\text { Atomic } \\
\text { Type }\end{array}$} & \multicolumn{3}{|c|}{ Coordinates (Angstroms) } \\
\hline & & & $\mathrm{X}$ & $\mathrm{Y}$ & Z \\
\hline 1 & 6 & 0 & 0.229759 & 0.794642 & -0.263747 \\
\hline 2 & 6 & 0 & 1.311573 & 0.046728 & -0.342258 \\
\hline 3 & 6 & 0 & -1.107731 & 0.205893 & 0.067764 \\
\hline 4 & 6 & 0 & 2.396648 & -0.682588 & -0.411577 \\
\hline 5 & 6 & 0 & 3.344847 & -0.946405 & 0.735171 \\
\hline 6 & 6 & 0 & -1.443285 & -1.010328 & -0.399957 \\
\hline 7 & 6 & 0 & -2.702869 & -1.790651 & -0.161568 \\
\hline 8 & 6 & 0 & -2.007039 & 1.062475 & 0.930543 \\
\hline 9 & 6 & 0 & 0.321600 & 2.292627 & -0.508412 \\
\hline 10 & 1 & 0 & 2.653226 & -1.147781 & -1.366284 \\
\hline 11 & 1 & 0 & 4.356011 & -0.594470 & 0.496247 \\
\hline 12 & 1 & 0 & 3.012764 & -0.445513 & 1.647454 \\
\hline 13 & 1 & 0 & 3.420732 & -2.021168 & 0.940559 \\
\hline 14 & 1 & 0 & -0.710413 & -1.508868 & -1.032745 \\
\hline 15 & 1 & 0 & -3.210156 & -2.011037 & -1.109924 \\
\hline 16 & 1 & 0 & -3.418657 & -1.279327 & 0.485520 \\
\hline 17 & 1 & 0 & -2.474170 & -2.761395 & 0.297707 \\
\hline 18 & 1 & 0 & -1.498545 & 1.353627 & 1.857802 \\
\hline 19 & 1 & 0 & -2.929590 & 0.549157 & 1.204728 \\
\hline 20 & 1 & 0 & -2.287387 & 1.991331 & 0.419678 \\
\hline 21 & 1 & 0 & -0.375100 & 2.604112 & -1.296240 \\
\hline 22 & 1 & 0 & 1.331882 & 2.573216 & -0.812544 \\
\hline 23 & 1 & 0 & 0.068381 & 2.863763 & 0.392267 \\
\hline
\end{tabular}

\section{Acetaldehyde}


$\mathrm{E}(\mathrm{RB}+\mathrm{HF}-\mathrm{LYP})=-153.835729731$ A.U.

Zero-point correction $=0.055599$ (Hartree/Particle)

\begin{tabular}{|c|c|c|c|c|c|}
\hline \multirow{2}{*}{$\begin{array}{l}\text { Center } \\
\text { Number }\end{array}$} & \multirow{2}{*}{$\begin{array}{l}\text { Atomic } \\
\text { Number }\end{array}$} & \multirow{2}{*}{$\begin{array}{c}\text { Atomic } \\
\text { Type }\end{array}$} & \multicolumn{3}{|c|}{ Coordinates (Angstroms) } \\
\hline & & & $\mathrm{X}$ & $\mathrm{Y}$ & Z \\
\hline 1 & 6 & 0 & 0.233499 & 0.399988 & 0.000015 \\
\hline 2 & 8 & 0 & 1.237299 & -0.276926 & -0.000005 \\
\hline 3 & 6 & 0 & -1.170204 & -0.149110 & -0.000005 \\
\hline 4 & 1 & 0 & 0.299942 & 1.512370 & -0.000016 \\
\hline 5 & 1 & 0 & -1.712455 & 0.219032 & -0.879703 \\
\hline 6 & 1 & 0 & -1.712447 & 0.218978 & 0.879714 \\
\hline 7 & 1 & 0 & -1.153203 & -1.240242 & -0.000014 \\
\hline
\end{tabular}

\section{$\mathbf{T S}_{\mathrm{AN}}$}

$\mathrm{E}(\mathrm{RB}+\mathrm{HF}-\mathrm{LYP})=-465.828427290$ A.U.

Zero-point correction= 0.232481 (Hartree/Particle)

Imaginary Frequency -- $\quad-469.6061 \quad \mathrm{~cm}-1$

\begin{tabular}{|c|c|c|c|c|c|}
\hline \multirow{2}{*}{$\begin{array}{l}\text { Center } \\
\text { Number }\end{array}$} & \multirow{2}{*}{$\begin{array}{l}\text { Atomic } \\
\text { Number }\end{array}$} & \multirow{2}{*}{$\begin{array}{l}\text { Atomic } \\
\text { Type }\end{array}$} & \multicolumn{3}{|c|}{ Coordinates (Angstroms) } \\
\hline & & & $\mathrm{x}$ & $\mathrm{Y}$ & $\mathrm{Z}$ \\
\hline 1 & 6 & 0 & 0.277243 & -0.650380 & 0.993374 \\
\hline 2 & 6 & 0 & 1.269834 & -0.094800 & 0.209041 \\
\hline 3 & 6 & 0 & -1.050848 & -0.831669 & 0.552693 \\
\hline 4 & 6 & 0 & 2.453554 & -0.345820 & -0.332260 \\
\hline 5 & 6 & 0 & 3.127504 & -1.695229 & -0.351372 \\
\hline 6 & 6 & 0 & -1.307787 & -0.638411 & -0.794467 \\
\hline 7 & 6 & 0 & -2.659205 & -0.568651 & -1.421101 \\
\hline 8 & 6 & 0 & -2.157282 & -1.033179 & 1.566548 \\
\hline 9 & 1 & 0 & 0.461377 & -0.707984 & 2.068527 \\
\hline 10 & 1 & 0 & 2.997960 & 0.460305 & -0.824635 \\
\hline 11 & 1 & 0 & 4.102505 & -1.647602 & 0.150489 \\
\hline 12 & 1 & 0 & 2.519641 & -2.454361 & 0.146249 \\
\hline 13 & 1 & 0 & 3.319238 & -2.025557 & -1.380054 \\
\hline 14 & 1 & 0 & -0.475840 & -0.720792 & -1.482077 \\
\hline 15 & 1 & 0 & -2.666827 & 0.242627 & -2.155266 \\
\hline 16 & 1 & 0 & -3.456695 & -0.391298 & -0.696665 \\
\hline 17 & 1 & 0 & -2.884552 & -1.498978 & -1.960300 \\
\hline 18 & 1 & 0 & -1.742686 & -1.281083 & 2.547742 \\
\hline 19 & 1 & 0 & -2.837363 & -1.841650 & 1.279918 \\
\hline 20 & 1 & 0 & -2.761569 & -0.125824 & 1.686347 \\
\hline 21 & 8 & 0 & -0.601917 & 1.461345 & -0.970779 \\
\hline 22 & 6 & 0 & 0.495434 & 1.696742 & -0.373658 \\
\hline 23 & 6 & 0 & 0.468761 & 2.526748 & 0.906680 \\
\hline 24 & 1 & 0 & 1.392223 & 1.863954 & -0.997238 \\
\hline 25 & 1 & 0 & 0.221726 & 3.564012 & 0.647326 \\
\hline 26 & 1 & 0 & 1.433106 & 2.521439 & 1.423291 \\
\hline 27 & 1 & 0 & -0.310161 & 2.159937 & 1.579706 \\
\hline
\end{tabular}

\section{$\mathbf{T S}_{\mathrm{AX}}$}

$\mathrm{E}(\mathrm{RB}+\mathrm{HF}-\mathrm{LYP})=-465.824973370 \quad \mathrm{~A} . \mathrm{U}$.

Zero-point correction $=0.232360$ (Hartree/Particle)

Imaginary Frequency -- $\quad-479.6297 \mathrm{~cm}-1$

$\begin{array}{llll}\text { Center } & \text { Atomic } & \text { Atomic } & \text { Coordinates (Angstroms) } \\ \text { Number } & \text { Number } & \text { Type } & \mathrm{Y}\end{array}$

1

6

0

$-0.061437-1.235579$

$-0.602496$ 


\begin{tabular}{|c|c|c|c|c|c|}
\hline 2 & 6 & 0 & -1.164203 & -0.437521 & -0.369014 \\
\hline 3 & 6 & 0 & 1.235839 & -0.974422 & -0.123258 \\
\hline 4 & 6 & 0 & -2.421317 & -0.520867 & 0.048875 \\
\hline 5 & 6 & 0 & -3.001941 & -1.692286 & 0.802591 \\
\hline 6 & 6 & 0 & 1.389682 & 0.059755 & 0.789259 \\
\hline 7 & 6 & 0 & 2.696495 & 0.598901 & 1.269705 \\
\hline 8 & 6 & 0 & 2.423443 & -1.660098 & -0.761627 \\
\hline 9 & 1 & 0 & -0.153378 & -1.972651 & -1.404131 \\
\hline 10 & 1 & 0 & -3.108319 & 0.306725 & -0.117838 \\
\hline 11 & 1 & 0 & -3.882937 & -2.087008 & 0.280048 \\
\hline 12 & 1 & 0 & -2.271860 & -2.497336 & 0.913671 \\
\hline 13 & 1 & 0 & -3.339699 & -1.392812 & 1.803144 \\
\hline 14 & 1 & 0 & 0.521703 & 0.347541 & 1.371264 \\
\hline 15 & 1 & 0 & 2.607027 & 1.674780 & 1.440991 \\
\hline 16 & 1 & 0 & 3.506244 & 0.428215 & 0.557259 \\
\hline 17 & 1 & 0 & 2.978927 & 0.133013 & 2.224115 \\
\hline 18 & 1 & 0 & 2.101864 & -2.522913 & -1.351627 \\
\hline 19 & 1 & 0 & 3.145783 & -2.015231 & -0.019533 \\
\hline 20 & 1 & 0 & 2.956005 & -0.980665 & -1.438637 \\
\hline 21 & 8 & 0 & 0.733823 & 1.586166 & -0.571828 \\
\hline 22 & 6 & 0 & -0.511279 & 1.539738 & -0.817682 \\
\hline 23 & 6 & 0 & -1.441112 & 2.411099 & 0.016078 \\
\hline 24 & 1 & 0 & -0.820523 & 1.394384 & -1.866098 \\
\hline 25 & 1 & 0 & -2.493893 & 2.285698 & -0.246994 \\
\hline 26 & 1 & 0 & -1.172615 & 3.457995 & -0.170101 \\
\hline 27 & 1 & 0 & -1.309944 & 2.218610 & 1.084497 \\
\hline
\end{tabular}

\section{$\mathrm{TS}_{\mathrm{BN}}$}

$\mathrm{E}(\mathrm{RB}+\mathrm{HF}-\mathrm{LYP})=-505.147770811 \quad \mathrm{~A} . \mathrm{U}$.

Zero-point correction $=0.260474$ (Hartree/Particle)

Imaginary Frequency -- $\quad-439.5277 \mathrm{~cm}-1$

\begin{tabular}{|c|c|c|c|c|c|}
\hline \multirow{2}{*}{$\begin{array}{l}\text { Center } \\
\text { Number }\end{array}$} & \multirow{2}{*}{$\begin{array}{l}\text { Atomic } \\
\text { Number }\end{array}$} & \multirow{2}{*}{$\begin{array}{l}\text { Atomic } \\
\text { Type }\end{array}$} & \multicolumn{3}{|c|}{ Coordinates (Angstroms) } \\
\hline & & & $\mathrm{X}$ & $\mathrm{Y}$ & Z \\
\hline 1 & 6 & 0 & 0.302233 & -0.833270 & 0.482522 \\
\hline 2 & 6 & 0 & 1.187628 & 0.070080 & -0.090195 \\
\hline 3 & 6 & 0 & -1.055178 & -0.886411 & 0.050305 \\
\hline 4 & 6 & 0 & 2.301105 & 0.107703 & -0.811359 \\
\hline 5 & 6 & 0 & 2.958911 & -1.084615 & -1.460929 \\
\hline 6 & 6 & 0 & -1.389621 & -0.163365 & -1.078715 \\
\hline 7 & 6 & 0 & -2.758590 & 0.107045 & -1.607213 \\
\hline 8 & 6 & 0 & -2.083707 & -1.589354 & 0.910155 \\
\hline 9 & 6 & 0 & 0.722701 & -1.538097 & 1.755252 \\
\hline 10 & 1 & 0 & 2.790413 & 1.066418 & -0.985855 \\
\hline 11 & 1 & 0 & 3.984742 & -1.212152 & -1.091744 \\
\hline 12 & 1 & 0 & 2.406032 & -2.006866 & -1.266584 \\
\hline 13 & 1 & 0 & 3.031691 & -0.950371 & -2.547617 \\
\hline 14 & 1 & 0 & -0.584197 & 0.120442 & -1.743334 \\
\hline 15 & 1 & 0 & -2.806427 & 1.155848 & -1.915366 \\
\hline 16 & 1 & 0 & -3.552043 & -0.073192 & -0.879772 \\
\hline 17 & 1 & 0 & -2.959903 & -0.503901 & -2.497663 \\
\hline 18 & 1 & 0 & -1.774119 & -2.616218 & 1.133422 \\
\hline 19 & 1 & 0 & -3.055315 & -1.647939 & 0.417887 \\
\hline 20 & 1 & 0 & -2.229731 & -1.084790 & 1.873363 \\
\hline 21 & 1 & 0 & 0.171627 & -1.165550 & 2.628170 \\
\hline 22 & 1 & 0 & 0.514063 & -2.612351 & 1.685720 \\
\hline 23 & 1 & 0 & 1.788731 & -1.402831 & 1.943206 \\
\hline 24 & 8 & 0 & -0.741067 & 1.899639 & -0.396021 \\
\hline 25 & 6 & 0 & 0.378726 & 1.883637 & 0.204393 \\
\hline
\end{tabular}




\begin{tabular}{|c|c|c|c|c|c|}
\hline 26 & 6 & 0 & 0.400329 & 2.083237 & 1.718470 \\
\hline 27 & 1 & 0 & 1.244828 & 2.342908 & -0.308452 \\
\hline 28 & 1 & 0 & 0.127917 & 3.123104 & 1.938007 \\
\hline 29 & 1 & 0 & 1.387115 & 1.888265 & 2.149464 \\
\hline 30 & 1 & 0 & -0.344118 & 1.442524 & 2.199190 \\
\hline
\end{tabular}

\section{$\mathbf{T S}_{\mathrm{BX}}$}

\begin{tabular}{|c|c|c|c|c|c|}
\hline \multirow{2}{*}{$\begin{array}{l}\text { Center } \\
\text { Number }\end{array}$} & \multirow{2}{*}{$\begin{array}{l}\text { Atomic } \\
\text { Number }\end{array}$} & \multirow{2}{*}{$\begin{array}{l}\text { Atomic } \\
\text { Type }\end{array}$} & \multicolumn{3}{|c|}{ Coordinates (Angstroms) } \\
\hline & & & $\mathrm{X}$ & $\mathrm{Y}$ & Z \\
\hline 1 & 6 & 0 & 0.081382 & -1.078778 & -0.365678 \\
\hline 2 & 6 & 0 & -1.065709 & -0.339236 & -0.096989 \\
\hline 3 & 6 & 0 & 1.353339 & -0.629999 & 0.081347 \\
\hline 4 & 6 & 0 & -2.194489 & -0.450272 & 0.591631 \\
\hline 5 & 6 & 0 & -2.500874 & -1.560009 & 1.569339 \\
\hline 6 & 6 & 0 & 1.371953 & 0.475376 & 0.914612 \\
\hline 7 & 6 & 0 & 2.571365 & 1.253459 & 1.343744 \\
\hline 8 & 6 & 0 & 2.612258 & -1.235340 & -0.500059 \\
\hline 9 & 6 & 0 & -0.016946 & -2.193916 & -1.386011 \\
\hline 10 & 1 & 0 & -2.971999 & 0.303953 & 0.492945 \\
\hline 11 & 1 & 0 & -3.413289 & -2.095617 & 1.276757 \\
\hline 12 & 1 & 0 & -1.681764 & -2.280594 & 1.631453 \\
\hline 13 & 1 & 0 & -2.682703 & -1.159666 & 2.574955 \\
\hline 14 & 1 & 0 & 0.461040 & 0.706988 & 1.451672 \\
\hline 15 & 1 & 0 & 2.311002 & 2.315425 & 1.359245 \\
\hline 16 & 1 & 0 & 3.430270 & 1.121182 & 0.683355 \\
\hline 17 & 1 & 0 & 2.872802 & 0.973409 & 2.362454 \\
\hline 18 & 1 & 0 & 2.588929 & -2.328730 & -0.441974 \\
\hline 19 & 1 & 0 & 3.506188 & -0.905640 & 0.031543 \\
\hline 20 & 1 & 0 & 2.740739 & -0.974735 & -1.558482 \\
\hline 21 & 1 & 0 & 0.478509 & -1.924190 & -2.327766 \\
\hline 22 & 1 & 0 & 0.478730 & -3.099944 & -1.018381 \\
\hline 23 & 1 & 0 & -1.058663 & -2.432232 & -1.605826 \\
\hline 24 & 8 & 0 & 0.385895 & 1.923671 & -0.441367 \\
\hline 25 & 6 & 0 & -0.682065 & 1.486687 & -0.977251 \\
\hline 26 & 6 & 0 & -1.975836 & 2.255321 & -0.730588 \\
\hline 27 & 1 & 0 & -0.618685 & 1.072876 & -2.002556 \\
\hline 28 & 1 & 0 & -2.854540 & 1.739331 & -1.127104 \\
\hline 29 & 1 & 0 & -1.890132 & 3.217069 & -1.249971 \\
\hline 30 & 1 & 0 & -2.109858 & 2.461986 & 0.334034 \\
\hline
\end{tabular}

$\mathbf{P}_{\mathrm{AN}}$

$\mathrm{E}(\mathrm{RB}+\mathrm{HF}-\mathrm{LYP})=-465.919858140 \quad \mathrm{~A} . \mathrm{U}$.

Zero-point correction $=0.238945$ (Hartree/Particle)

\begin{tabular}{|c|c|c|c|c|c|}
\hline \multirow{2}{*}{$\begin{array}{l}\text { Center } \\
\text { Number }\end{array}$} & \multirow{2}{*}{$\begin{array}{l}\text { Atomic } \\
\text { Number }\end{array}$} & \multirow{2}{*}{$\begin{array}{c}\text { Atomic } \\
\text { Type }\end{array}$} & \multicolumn{3}{|c|}{ Coordinates (Angstroms) } \\
\hline & & & $\mathrm{X}$ & $\mathrm{Y}$ & $\mathrm{Z}$ \\
\hline 1 & 6 & 0 & 0.142303 & -1.200512 & -0.136069 \\
\hline 2 & 6 & 0 & 1.144671 & -0.134308 & -0.128997 \\
\hline 3 & 6 & 0 & -1.180989 & -0.963644 & -0.208668 \\
\hline 4 & 6 & 0 & 2.458079 & -0.327018 & 0.101308 \\
\hline 5 & 6 & 0 & 3.150761 & -1.617165 & 0.434261 \\
\hline 6 & 6 & 0 & -1.697520 & 0.464027 & -0.191619 \\
\hline 7 & 6 & 0 & -2.857724 & 0.687225 & 0.779608 \\
\hline
\end{tabular}




$\begin{array}{rrrrrr}8 & 6 & 0 & -2.189933 & -2.071482 & -0.345214 \\ 9 & 1 & 0 & 0.485831 & -2.231830 & -0.123469 \\ 10 & 1 & 0 & 3.117746 & 0.536166 & 0.048849 \\ 11 & 1 & 0 & 3.650795 & -1.545748 & 1.408930 \\ 12 & 1 & 0 & 2.475279 & -2.473901 & 0.475127 \\ 13 & 1 & 0 & 3.935593 & -1.840604 & -0.299997 \\ 14 & 1 & 0 & -2.049504 & 0.707521 & -1.215136 \\ 15 & 1 & 0 & -3.121804 & 1.747815 & 0.785808 \\ 16 & 1 & 0 & -2.565278 & 0.398259 & 1.793376 \\ 17 & 1 & 0 & -3.740318 & 0.112281 & 0.487545 \\ 18 & 1 & 0 & -1.697090 & -3.040998 & -0.456920 \\ 19 & 1 & 0 & -2.832183 & -1.918038 & -1.222971 \\ 20 & 1 & 0 & -2.857368 & -2.128869 & 0.523184 \\ 21 & 8 & 0 & -0.691834 & 1.394654 & 0.187234 \\ 22 & 6 & 0 & 0.558411 & 1.224441 & -0.491113 \\ 23 & 6 & 0 & 1.399433 & 2.443399 & -0.140273 \\ 24 & 1 & 0 & 0.364077 & 1.226742 & -1.579919 \\ 25 & 1 & 0 & 0.833846 & 3.348141 & -0.376632 \\ 26 & 1 & 0 & 2.331197 & 2.463208 & -0.711705 \\ 27 & 1 & 0 & 1.638901 & 2.452842 & 0.926717 \\ ------------------------------------------------------\end{array}$

\section{$\mathbf{P}_{\mathrm{AX}}$}

$\mathrm{E}(\mathrm{RB}+\mathrm{HF}-\mathrm{LYP})=-465.919117295 \quad$ A.U.

Zero-point correction $=0.238892$ (Hartree/Particle)

\begin{tabular}{|c|c|c|c|c|c|}
\hline \multirow{2}{*}{$\begin{array}{l}\text { Center } \\
\text { Number }\end{array}$} & \multirow{2}{*}{$\begin{array}{l}\text { Atomic } \\
\text { Number }\end{array}$} & \multirow{2}{*}{$\begin{array}{l}\text { Atomic } \\
\text { Type }\end{array}$} & \multicolumn{3}{|c|}{ Coordinates (Angstroms) } \\
\hline & & & $\mathrm{X}$ & $\mathrm{Y}$ & Z \\
\hline 1 & 6 & 0 & 0.370144 & 1.050634 & 0.220301 \\
\hline 2 & 6 & 0 & 1.217587 & -0.086165 & -0.126230 \\
\hline 3 & 6 & 0 & -0.971672 & 0.961399 & 0.297663 \\
\hline 4 & 6 & 0 & 2.537275 & -0.024376 & -0.388371 \\
\hline 5 & 6 & 0 & 3.417670 & 1.190854 & -0.401902 \\
\hline 6 & 6 & 0 & -1.675034 & -0.338751 & -0.044360 \\
\hline 7 & 6 & 0 & -2.852695 & -0.161686 & -1.005901 \\
\hline 8 & 6 & 0 & -1.825786 & 2.112348 & 0.756056 \\
\hline 9 & 1 & 0 & 0.845231 & 2.001331 & 0.450513 \\
\hline 10 & 1 & 0 & 3.043709 & -0.962098 & -0.621224 \\
\hline 11 & 1 & 0 & 3.880155 & 1.324808 & -1.388396 \\
\hline 12 & 1 & 0 & 2.880445 & 2.111215 & -0.163200 \\
\hline 13 & 1 & 0 & 4.242343 & 1.086448 & 0.315140 \\
\hline 14 & 1 & 0 & -2.068201 & -0.764941 & 0.899183 \\
\hline 15 & 1 & 0 & -3.263970 & -1.143843 & -1.252537 \\
\hline 16 & 1 & 0 & -2.517342 & 0.314020 & -1.932139 \\
\hline 17 & 1 & 0 & -3.646346 & 0.444288 & -0.560980 \\
\hline 18 & 1 & 0 & -1.209212 & 2.948043 & 1.097775 \\
\hline 19 & 1 & 0 & -2.481448 & 1.815923 & 1.585789 \\
\hline 20 & 1 & 0 & -2.481093 & 2.480398 & -0.042716 \\
\hline 21 & 8 & 0 & -0.815500 & -1.283751 & -0.675796 \\
\hline 22 & 6 & 0 & 0.492776 & -1.421957 & -0.111655 \\
\hline 23 & 6 & 0 & 0.450501 & -2.053408 & 1.287576 \\
\hline 24 & 1 & 0 & 0.999015 & -2.116390 & -0.790552 \\
\hline 25 & 1 & 0 & 1.463960 & -2.295413 & 1.621073 \\
\hline 26 & 1 & 0 & -0.140749 & -2.974286 & 1.265580 \\
\hline 27 & 1 & 0 & 0.012905 & -1.372844 & 2.024008 \\
\hline
\end{tabular}

$\mathbf{P}_{\text {BN }}$

$E(R B+H F-L Y P)=-505.225651241 \quad$ A.U.

Zero-point correction= 0.266402 (Hartree/Particle) 


\begin{tabular}{|c|c|c|c|c|c|}
\hline \multirow{2}{*}{$\begin{array}{l}\text { Center } \\
\text { Number }\end{array}$} & \multirow{2}{*}{$\begin{array}{l}\text { Atomic } \\
\text { Number }\end{array}$} & \multirow{2}{*}{$\begin{array}{c}\text { Atomic } \\
\text { Type }\end{array}$} & \multicolumn{3}{|c|}{ Coordinates (Angstroms) } \\
\hline & & & $\mathrm{X}$ & $\mathrm{Y}$ & $\mathrm{Z}$ \\
\hline 1 & 6 & 0 & 0.341374 & -0.873492 & 0.297533 \\
\hline 2 & 6 & 0 & 1.047799 & 0.353081 & -0.142867 \\
\hline 3 & 6 & 0 & -0.976696 & -0.949363 & 0.005375 \\
\hline 4 & 6 & 0 & 2.282929 & 0.418399 & -0.672638 \\
\hline 5 & 6 & 0 & 3.247450 & -0.694022 & -0.971777 \\
\hline 6 & 6 & 0 & -1.588709 & 0.261493 & -0.693825 \\
\hline 7 & 6 & 0 & -3.111662 & 0.326683 & -0.699084 \\
\hline 8 & 6 & 0 & -1.844754 & -2.138695 & 0.315418 \\
\hline 9 & 6 & 0 & 1.090107 & -1.948869 & 1.064369 \\
\hline 10 & 1 & 0 & 2.640047 & 1.410451 & -0.954874 \\
\hline 11 & 1 & 0 & 4.111266 & -0.675086 & -0.293077 \\
\hline 12 & 1 & 0 & 2.792022 & -1.682803 & -0.907083 \\
\hline 13 & 1 & 0 & 3.650221 & -0.578621 & -1.985387 \\
\hline 14 & 1 & 0 & -1.235929 & 0.284529 & -1.741061 \\
\hline 15 & 1 & 0 & -3.430300 & 1.244847 & -1.198573 \\
\hline 16 & 1 & 0 & -3.501982 & 0.344277 & 0.322632 \\
\hline 17 & 1 & 0 & -3.547588 & -0.524550 & -1.228985 \\
\hline 18 & 1 & 0 & -1.285503 & -2.943413 & 0.794939 \\
\hline 19 & 1 & 0 & -2.290023 & -2.551487 & -0.599182 \\
\hline 20 & 1 & 0 & -2.681210 & -1.871493 & 0.972672 \\
\hline 21 & 1 & 0 & 0.503091 & -2.312816 & 1.913244 \\
\hline 22 & 1 & 0 & 1.331778 & -2.821195 & 0.444327 \\
\hline 23 & 1 & 0 & 2.029792 & -1.559138 & 1.461059 \\
\hline 24 & 8 & 0 & -1.155501 & 1.454648 & -0.029374 \\
\hline 25 & 6 & 0 & 0.255975 & 1.657155 & 0.008597 \\
\hline 26 & 6 & 0 & 0.551016 & 2.363743 & 1.333697 \\
\hline 27 & 1 & 0 & 0.535648 & 2.324778 & -0.822743 \\
\hline 28 & 1 & 0 & -0.082943 & 3.250467 & 1.430530 \\
\hline 29 & 1 & 0 & 1.599264 & 2.671968 & 1.384753 \\
\hline 30 & 1 & 0 & 0.337376 & 1.695428 & 2.173017 \\
\hline
\end{tabular}

$\mathbf{P}_{\mathbf{B X}}$

$\mathrm{E}(\mathrm{RB}+\mathrm{HF}-\mathrm{LYP})=-505.222888163$ A.U.

Zero-point correction $=0.266447$ (Hartree/Particle)

\begin{tabular}{|c|c|c|c|c|c|}
\hline \multirow{2}{*}{$\begin{array}{l}\text { Center } \\
\text { Number }\end{array}$} & \multirow{2}{*}{$\begin{array}{l}\text { Atomic } \\
\text { Number }\end{array}$} & \multirow{2}{*}{$\begin{array}{c}\text { Atomic } \\
\text { Type }\end{array}$} & \multicolumn{3}{|c|}{ Coordinates (Angstroms) } \\
\hline & & & $\mathrm{X}$ & $\mathrm{Y}$ & Z \\
\hline 1 & 6 & 0 & -0.217536 & -1.035191 & -0.305264 \\
\hline 2 & 6 & 0 & -1.058288 & 0.183025 & -0.160455 \\
\hline 3 & 6 & 0 & 1.084332 & -0.905506 & 0.027679 \\
\hline 4 & 6 & 0 & -2.292679 & 0.217996 & 0.371815 \\
\hline 5 & 6 & 0 & -3.064251 & -0.917178 & 0.984649 \\
\hline 6 & 6 & 0 & 1.509560 & 0.463795 & 0.546439 \\
\hline 7 & 6 & 0 & 3.012247 & 0.708664 & 0.626256 \\
\hline 8 & 6 & 0 & 2.093513 & -2.019661 & -0.040088 \\
\hline 9 & 6 & 0 & -0.820459 & -2.307758 & -0.872393 \\
\hline 10 & 1 & 0 & -2.809463 & 1.174453 & 0.395126 \\
\hline 11 & 1 & 0 & -3.918392 & -1.210770 & 0.359381 \\
\hline 12 & 1 & 0 & -2.452140 & -1.803452 & 1.158038 \\
\hline 13 & 1 & 0 & -3.482110 & -0.606687 & 1.949953 \\
\hline 14 & 1 & 0 & 1.086790 & 0.588537 & 1.559458 \\
\hline 15 & 1 & 0 & 3.191282 & 1.726900 & 0.980334 \\
\hline 16 & 1 & 0 & 3.476478 & 0.607005 & -0.358969 \\
\hline 17 & 1 & 0 & 3.494430 & 0.012886 & 1. 318628 \\
\hline 18 & 1 & 0 & 1.657549 & -2.946764 & -0.416065 \\
\hline
\end{tabular}




\begin{tabular}{|c|c|c|c|c|c|}
\hline 19 & 1 & 0 & 2.514128 & -2.235217 & 0.950800 \\
\hline 20 & 1 & 0 & 2.940922 & -1.759992 & -0.685867 \\
\hline 21 & 1 & 0 & -0.181736 & -2.738901 & -1.649941 \\
\hline 22 & 1 & 0 & -0.964576 & -3.083334 & -0.109983 \\
\hline 23 & 1 & 0 & -1.794721 & -2.108210 & -1.323587 \\
\hline 24 & 8 & 0 & 1.003627 & 1.511779 & -0.292884 \\
\hline 25 & 6 & 0 & -0.374177 & 1.437636 & -0.699301 \\
\hline 26 & 6 & 0 & -1.023153 & 2.773733 & -0.345939 \\
\hline 27 & 1 & 0 & -0.360174 & 1.343434 & -1.797591 \\
\hline 28 & 1 & 0 & -2.011350 & 2.877498 & -0.804510 \\
\hline 29 & 1 & 0 & -0.389527 & 3.581855 & -0.720180 \\
\hline 30 & 1 & 0 & -1.121066 & 2.889186 & 0.737658 \\
\hline
\end{tabular}

\section{C alpha}

$\mathrm{E}(\mathrm{RB}+\mathrm{HF}-\mathrm{LYP})=-390.660180210 \quad$ A.U.

Zero-point correction $=0.231026$ (Hartree/Particle)

\begin{tabular}{|c|c|c|c|c|c|}
\hline \multirow{2}{*}{$\begin{array}{l}\text { Center } \\
\text { Number }\end{array}$} & \multirow{2}{*}{$\begin{array}{l}\text { Atomic } \\
\text { Number }\end{array}$} & \multirow{2}{*}{$\begin{array}{c}\text { Atomic } \\
\text { Type }\end{array}$} & \multicolumn{3}{|c|}{ Coordinates (Angstroms) } \\
\hline & & & $\mathrm{x}$ & $\mathrm{Y}$ & Z \\
\hline 1 & 6 & 0 & 0.238327 & 0.525694 & 0.078704 \\
\hline 2 & 6 & 0 & 1.373961 & -0.065466 & -0.229936 \\
\hline 3 & 6 & 0 & -1.020480 & -0.266569 & 0.271468 \\
\hline 4 & 6 & 0 & 2.516140 & -0.638744 & -0.515519 \\
\hline 5 & 6 & 0 & 3.534037 & -1.106215 & 0.498763 \\
\hline 6 & 6 & 0 & -1.313619 & -1.275906 & -0.568375 \\
\hline 7 & 6 & 0 & -2.484079 & -2.214927 & -0.530976 \\
\hline 8 & 6 & 0 & -1.882447 & 0.142324 & 1.445327 \\
\hline 9 & 6 & 0 & 0.183783 & 2.043669 & 0.228912 \\
\hline 10 & 6 & 0 & -0.677482 & 2.720903 & -0.848283 \\
\hline 11 & 1 & 0 & 2.769649 & -0.803188 & -1.565232 \\
\hline 12 & 1 & 0 & 4.498577 & -0.606814 & 0.344878 \\
\hline 13 & 1 & 0 & 3.200038 & -0.902097 & 1.518706 \\
\hline 14 & 1 & 0 & 3.713644 & -2.184188 & 0.404739 \\
\hline 15 & 1 & 0 & -0.613456 & -1.450301 & -1.383988 \\
\hline 16 & 1 & 0 & -3.065957 & -2.149602 & -1.459519 \\
\hline 17 & 1 & 0 & -3.165978 & -2.029164 & 0.301299 \\
\hline 18 & 1 & 0 & -2.141676 & -3.255319 & -0.454652 \\
\hline 19 & 1 & 0 & -1.299576 & 0.141742 & 2.374404 \\
\hline 20 & 1 & 0 & -2.734668 & -0.524023 & 1.585876 \\
\hline 21 & 1 & 0 & -2.277676 & 1.157524 & 1.321609 \\
\hline 22 & 1 & 0 & 1.203483 & 2.437891 & 0.187911 \\
\hline 23 & 1 & 0 & -0.207741 & 2.299605 & 1.222060 \\
\hline 24 & 1 & 0 & -0.716830 & 3.804353 & -0.693833 \\
\hline 25 & 1 & 0 & -1.704155 & 2.340994 & -0.839356 \\
\hline 26 & 1 & 0 & -0.266532 & 2.534011 & -1.845399 \\
\hline
\end{tabular}

\section{C beta}

$\mathrm{E}(\mathrm{RB}+\mathrm{HF}-\mathrm{LYP})=-390.658353900 \quad$ A.U.

Zero-point correction $=0.231090$ (Hartree/Particle)

\begin{tabular}{|c|c|c|c|c|c|}
\hline \multirow{2}{*}{$\begin{array}{l}\text { Center } \\
\text { Number }\end{array}$} & \multirow{2}{*}{$\begin{array}{l}\text { Atomic } \\
\text { Number }\end{array}$} & \multirow{2}{*}{$\begin{array}{l}\text { Atomic } \\
\text { Type }\end{array}$} & \multicolumn{3}{|c|}{ Coordinates (Angstroms) } \\
\hline & & & $\mathrm{X}$ & $\mathrm{Y}$ & $\mathrm{Z}$ \\
\hline 1 & 6 & 0 & 0.215271 & 0.376771 & -0.402650 \\
\hline 2 & 6 & 0 & 1.122330 & -0.579147 & -0.393540 \\
\hline 3 & 6 & 0 & -1.196426 & 0.101655 & 0.020370 \\
\hline 4 & 6 & 0 & 2.040909 & -1.512375 & -0.385544 \\
\hline 5 & 6 & 0 & 2.977692 & -1.805023 & 0.763488 \\
\hline
\end{tabular}




$\begin{array}{rrrrrr}6 & 6 & 0 & -1.791749 & -1.044509 & -0.358369 \\ 7 & 6 & 0 & -3.166733 & -1.542159 & -0.019929 \\ 8 & 6 & 0 & -1.864593 & 1.160813 & 0.867538 \\ 9 & 6 & 0 & 0.611222 & 1.784883 & -0.856171 \\ 10 & 6 & 0 & 1.203874 & 2.663956 & 0.256896 \\ 11 & 1 & 0 & 2.150986 & -2.144074 & -1.270157 \\ 12 & 1 & 0 & 4.024338 & -1.697540 & 0.453094 \\ 13 & 1 & 0 & 2.796967 & -1.130722 & 1.603866 \\ 14 & 1 & 0 & 2.853256 & -2.836029 & 1.116531 \\ 15 & 1 & 0 & -1.206680 & -1.713668 & -0.987473 \\ 16 & 1 & 0 & -3.751221 & -1.725158 & -0.930939 \\ 17 & 1 & 0 & -3.737747 & -0.856765 & 0.609924 \\ 18 & 1 & 0 & -3.108566 & -2.503921 & 0.506744 \\ 19 & 1 & 0 & -1.352402 & 1.271714 & 1.831329 \\ 20 & 1 & 0 & -2.911209 & 0.930039 & 1.071639 \\ 21 & 1 & 0 & -1.835219 & 2.141724 & 0.380294 \\ 22 & 1 & 0 & -0.262893 & 2.279817 & -1.296342 \\ 23 & 1 & 0 & 1.348664 & 1.691147 & -1.659913 \\ 24 & 1 & 0 & 1.484681 & 3.648193 & -0.133683 \\ 25 & 1 & 0 & 2.100358 & 2.196808 & 0.676315 \\ 26 & 1 & 0 & 0.495896 & 2.819245 & 1.076217 \\ -------------------------------------------------------------\end{array}$

\section{Acetaldehyde- $\mathrm{BF}_{3}$}

$\mathrm{E}(\mathrm{RB}+\mathrm{HF}-\mathrm{LYP})=-478.407099530$ A.U.

Zero-point correction $=0.070794$ (Hartree/Particle)

\begin{tabular}{|c|c|c|c|c|c|}
\hline \multirow{2}{*}{$\begin{array}{l}\text { Center } \\
\text { Number }\end{array}$} & \multirow{2}{*}{$\begin{array}{l}\text { Atomic } \\
\text { Number }\end{array}$} & \multirow{2}{*}{$\begin{array}{l}\text { Atomic } \\
\text { Type }\end{array}$} & \multicolumn{3}{|c|}{ Coordinates (Angstroms) } \\
\hline & & & $\mathrm{x}$ & $\mathrm{Y}$ & $\mathrm{Z}$ \\
\hline 1 & 9 & 0 & -1.552696 & -0.527461 & -1.151902 \\
\hline 2 & 5 & 0 & -1.109351 & 0.015447 & -0.000005 \\
\hline 3 & 9 & 0 & -1.552599 & -0.527377 & 1.151996 \\
\hline 4 & 9 & 0 & -0.957740 & 1.371595 & -0.000056 \\
\hline 5 & 8 & 0 & 0.568317 & -0.537999 & -0.000045 \\
\hline 6 & 6 & 0 & 1.493832 & 0.272400 & 0.000002 \\
\hline 7 & 6 & 0 & 2.917535 & -0.151941 & 0.000002 \\
\hline 8 & 1 & 0 & 1.253476 & 1.344581 & 0.000034 \\
\hline 9 & 1 & 0 & 3.419545 & 0.273476 & 0.877776 \\
\hline 10 & 1 & 0 & 3.419610 & 0.273621 & -0.877691 \\
\hline 11 & 1 & 0 & 3.006697 & -1.238483 & -0.000103 \\
\hline
\end{tabular}

\section{cTS $_{\mathrm{AN}}$}

$\mathrm{E}(\mathrm{RB}+\mathrm{HF}-\mathrm{LYP})=-790.424787819 \quad \mathrm{~A} . \mathrm{U}$.

Zero-point correction $=0.247640$ (Hartree/Particle)

Imaginary Frequency -- $-301.8323 \mathrm{~cm}-1$

\begin{tabular}{|c|c|c|c|c|c|}
\hline \multirow{2}{*}{$\begin{array}{l}\text { Center } \\
\text { Number }\end{array}$} & \multirow{2}{*}{$\begin{array}{l}\text { Atomic } \\
\text { Number }\end{array}$} & \multirow{2}{*}{$\begin{array}{l}\text { Atomic } \\
\text { Type }\end{array}$} & \multicolumn{3}{|c|}{ Coordinates (Angstroms) } \\
\hline & & & $\mathrm{X}$ & $\mathrm{Y}$ & Z \\
\hline 1 & 6 & 0 & -1.796712 & -2.864048 & -1.729405 \\
\hline 2 & 6 & 0 & -0.898403 & -2.387725 & -0.616447 \\
\hline 3 & 6 & 0 & -1.129697 & -1.320279 & 0.125391 \\
\hline 4 & 6 & 0 & -1.975800 & -0.293677 & 0.447606 \\
\hline 5 & 6 & 0 & -1.820633 & 1.081575 & 0.077123 \\
\hline 6 & 6 & 0 & -2.696272 & 2.114797 & 0.757323 \\
\hline 7 & 6 & 0 & -0.844214 & 1.412992 & -0.825008 \\
\hline 8 & 6 & 0 & -0.440352 & 2.781813 & -1.237443 \\
\hline 9 & 1 & 0 & -2.104704 & -3.902529 & -1.557528 \\
\hline 10 & 1 & 0 & -2.692276 & -2.245589 & -1.821517 \\
\hline
\end{tabular}




$\begin{array}{rrrrrr}11 & 1 & 0 & -1.259824 & -2.843332 & -2.685044 \\ 12 & 1 & 0 & 0.013509 & -2.963059 & -0.466987 \\ 13 & 1 & 0 & -2.743223 & -0.505160 & 1.197122 \\ 14 & 1 & 0 & -3.545342 & 1.642120 & 1.258875 \\ 15 & 1 & 0 & -3.097343 & 2.839705 & 0.043664 \\ 16 & 1 & 0 & -2.138355 & 2.677204 & 1.515046 \\ 17 & 1 & 0 & -0.289301 & 0.604724 & -1.292079 \\ 18 & 1 & 0 & 0.634917 & 2.880733 & -1.044024 \\ 19 & 1 & 0 & -0.982840 & 3.578476 & -0.725698 \\ 20 & 1 & 0 & -0.565532 & 2.903981 & -2.321774 \\ 21 & 6 & 0 & 0.531506 & -0.855403 & 1.306056 \\ 22 & 6 & 0 & 0.034721 & -0.839757 & 2.727678 \\ 23 & 8 & 0 & 1.033412 & 0.240831 & 0.857793 \\ 24 & 5 & 0 & 2.158314 & 0.162672 & -0.260088 \\ 25 & 9 & 0 & 3.202857 & -0.514677 & 0.319392 \\ 26 & 9 & 0 & 1.607572 & -0.532361 & -1.330478 \\ 27 & 9 & 0 & 2.426091 & 1.479195 & -0.554504 \\ 28 & 1 & 0 & 0.984128 & -1.787313 & 0.957193 \\ 29 & 1 & 0 & 0.913780 & -0.872815 & 3.383840 \\ 30 & 1 & 0 & -0.595304 & -1.703537 & 2.949628 \\ 31 & 1 & 0 & -0.504696 & 0.085229 & 2.940449 \\ ------------------------------------------------------\end{array}$

\section{$\operatorname{cTS}_{\mathrm{AX}}$}

$\mathrm{E}(\mathrm{RB}+\mathrm{HF}-\mathrm{LYP})=-790.421626180 \quad \mathrm{~A} . \mathrm{U}$.

Zero-point correction= 0.247852 (Hartree/Particle)

Imaginary Frequency -- $\quad-298.2010 \mathrm{~cm}-1$

\begin{tabular}{|c|c|c|c|c|c|}
\hline \multirow{2}{*}{$\begin{array}{l}\text { Center } \\
\text { Number }\end{array}$} & \multirow{2}{*}{$\begin{array}{l}\text { Atomic } \\
\text { Number }\end{array}$} & \multirow{2}{*}{$\begin{array}{c}\text { Atomic } \\
\text { Type }\end{array}$} & \multicolumn{3}{|c|}{ Coordinates (Angstroms) } \\
\hline & & & $\mathrm{X}$ & $\mathrm{Y}$ & $\mathrm{Z}$ \\
\hline 1 & 9 & 0 & -2.579669 & 0.287665 & 0.444613 \\
\hline 2 & 5 & 0 & -1.979321 & -0.779686 & -0.194433 \\
\hline 3 & 9 & 0 & -1.578841 & -0.461082 & -1.492554 \\
\hline 4 & 9 & 0 & -2.718457 & -1.933667 & -0.146135 \\
\hline 5 & 8 & 0 & -0.665408 & -1.084353 & 0.620742 \\
\hline 6 & 6 & 0 & 0.293984 & -1.693694 & 0.004650 \\
\hline 7 & 6 & 0 & 1.053106 & -2.711423 & 0.812119 \\
\hline 8 & 1 & 0 & 0.130890 & -1.925198 & -1.048889 \\
\hline 9 & 1 & 0 & 1.901033 & -3.127025 & 0.266858 \\
\hline 10 & 1 & 0 & 0.345231 & -3.525613 & 1.010484 \\
\hline 11 & 1 & 0 & 1.376570 & -2.307736 & 1.773858 \\
\hline 12 & 6 & 0 & 1.051481 & 0.721633 & -1.018101 \\
\hline 13 & 6 & 0 & 1.793333 & -0.264361 & -0.411907 \\
\hline 14 & 6 & 0 & 0.181596 & 1.644265 & -0.361314 \\
\hline 15 & 6 & 0 & 3.021016 & -0.559851 & -0.015306 \\
\hline 16 & 6 & 0 & 4.195867 & 0.383615 & -0.110459 \\
\hline 17 & 6 & 0 & 0.112966 & 1.618446 & 1.006921 \\
\hline 18 & 6 & 0 & -0.823507 & 2.412353 & 1.844864 \\
\hline 19 & 6 & 0 & -0.720322 & 2.524538 & -1.194990 \\
\hline 20 & 1 & 0 & 0.988854 & 0.696332 & -2.107694 \\
\hline 21 & 1 & 0 & 3.240416 & -1.530567 & 0.420622 \\
\hline 22 & 1 & 0 & 4.988987 & -0.052850 & -0.729996 \\
\hline 23 & 1 & 0 & 3.909025 & 1.345343 & -0.541165 \\
\hline 24 & 1 & 0 & 4.631486 & 0.563123 & 0.879949 \\
\hline 25 & 1 & 0 & 0.774552 & 0.933840 & 1.529153 \\
\hline 26 & 1 & 0 & -1.719353 & 1.797725 & 2.006687 \\
\hline 27 & 1 & 0 & -1.147839 & 3.336356 & 1.360158 \\
\hline 28 & 1 & 0 & -0.390536 & 2.644689 & 2.822055 \\
\hline 29 & 1 & 0 & -0.459969 & 2.467266 & -2.254411 \\
\hline 30 & 1 & 0 & -0.657369 & 3.573877 & -0.889230 \\
\hline
\end{tabular}


$\mathbf{c P}_{\mathrm{AN}}$

$\mathrm{E}(\mathrm{RB}+\mathrm{HF}-\mathrm{LYP})=-790.493930878 \quad$ A.U.

Zero-point correction $=0.254084$ (Hartree/Particle)

\begin{tabular}{|c|c|c|c|c|c|}
\hline \multirow{2}{*}{$\begin{array}{l}\text { Center } \\
\text { Number }\end{array}$} & \multirow{2}{*}{$\begin{array}{l}\text { Atomic } \\
\text { Number }\end{array}$} & \multirow{2}{*}{$\begin{array}{c}\text { Atomic } \\
\text { Type }\end{array}$} & \multicolumn{3}{|c|}{ Coordinates (Angstroms) } \\
\hline & & & $\mathrm{X}$ & $\mathrm{Y}$ & $\mathrm{Z}$ \\
\hline 1 & 9 & 0 & 2.707379 & 0.050992 & -1.387950 \\
\hline 2 & 5 & 0 & 2.175338 & -0.830635 & -0.495692 \\
\hline 3 & 9 & 0 & 2.812274 & -0.871889 & 0.715650 \\
\hline 4 & 9 & 0 & 1.834497 & -2.049918 & -0.996453 \\
\hline 5 & 6 & 0 & -1.896225 & 0.848700 & -0.177217 \\
\hline 6 & 6 & 0 & -1.698519 & -0.579201 & 0.041350 \\
\hline 7 & 6 & 0 & -0.888942 & 1.739511 & -0.231226 \\
\hline 8 & 6 & 0 & -2.641429 & -1.529421 & -0.110822 \\
\hline 9 & 6 & 0 & -4.073067 & -1.349321 & -0.517295 \\
\hline 10 & 6 & 0 & 0.556990 & 1.319611 & -0.147391 \\
\hline 11 & 6 & 0 & 1.340167 & 1.939093 & 1.017004 \\
\hline 12 & 6 & 0 & -1.127243 & 3.211434 & -0.439697 \\
\hline 13 & 8 & 0 & 0.673898 & -0.140451 & -0.161766 \\
\hline 14 & 6 & 0 & -0.323847 & -0.970531 & 0.532523 \\
\hline 15 & 6 & 0 & -0.161477 & -0.900016 & 2.051324 \\
\hline 16 & 1 & 0 & -2.911452 & 1.211101 & -0.311434 \\
\hline 17 & 1 & 0 & -2.343859 & -2.560844 & 0.079597 \\
\hline 18 & 1 & 0 & -4.748254 & -1.755366 & 0.246338 \\
\hline 19 & 1 & 0 & -4.345741 & -0.306138 & -0.688346 \\
\hline 20 & 1 & 0 & -4.281362 & -1.902965 & -1.441543 \\
\hline 21 & 1 & 0 & 1.056524 & 1.599567 & -1.079277 \\
\hline 22 & 1 & 0 & 0.843640 & 1.778608 & 1.976138 \\
\hline 23 & 1 & 0 & 1.433527 & 3.016899 & 0.856066 \\
\hline 24 & 1 & 0 & 2.341941 & 1.510659 & 1.062134 \\
\hline 25 & 1 & 0 & -2.185774 & 3.411795 & -0.622353 \\
\hline 26 & 1 & 0 & -0.555285 & 3.591588 & -1.295662 \\
\hline 27 & 1 & 0 & -0.820341 & 3.801878 & 0.432222 \\
\hline 28 & 1 & 0 & -0.067772 & -1.969719 & 0.182237 \\
\hline 29 & 1 & 0 & 0.885720 & -1.046270 & 2.324037 \\
\hline 30 & 1 & 0 & -0.758784 & -1.696862 & 2.504130 \\
\hline 31 & 1 & 0 & -0.516401 & 0.051037 & 2.455758 \\
\hline
\end{tabular}

\section{$\mathbf{c P}_{\mathrm{AX}}$}

$\mathrm{E}(\mathrm{RB}+\mathrm{HF}-\mathrm{LYP})=-790.493930878 \quad \mathrm{~A} . \mathrm{U}$.

Zero-point correction $=0.254084$ (Hartree/Particle)

\begin{tabular}{|c|c|c|c|c|c|}
\hline \multirow{2}{*}{$\begin{array}{l}\text { Center } \\
\text { Number }\end{array}$} & \multirow{2}{*}{$\begin{array}{l}\text { Atomic } \\
\text { Number }\end{array}$} & \multirow{2}{*}{$\begin{array}{l}\text { Atomic } \\
\text { Type }\end{array}$} & \multicolumn{3}{|c|}{ Coordinates (Angstroms) } \\
\hline & & & $\mathrm{X}$ & $\mathrm{Y}$ & $\mathrm{Z}$ \\
\hline 1 & 9 & 0 & 2.707379 & 0.050992 & -1.387950 \\
\hline 2 & 5 & 0 & 2.175338 & -0.830635 & -0.495692 \\
\hline 3 & 9 & 0 & 2.812274 & -0.871889 & 0.715650 \\
\hline 4 & 9 & 0 & 1.834497 & -2.049918 & -0.996453 \\
\hline 5 & 6 & 0 & -1.896225 & 0.848700 & -0.177217 \\
\hline 6 & 6 & 0 & -1.698519 & -0.579201 & 0.041350 \\
\hline 7 & 6 & 0 & -0.888942 & 1.739511 & -0.231226 \\
\hline 8 & 6 & 0 & -2.641429 & -1.529421 & -0.110822 \\
\hline 9 & 6 & 0 & -4.073067 & -1.349321 & -0.517295 \\
\hline 10 & 6 & 0 & 0.556990 & 1.319611 & -0.147391 \\
\hline 11 & 6 & 0 & 1.340167 & 1.939093 & 1.017004 \\
\hline
\end{tabular}




$\begin{array}{rrrrrr}12 & 6 & 0 & -1.127243 & 3.211434 & -0.439697 \\ 13 & 8 & 0 & 0.673898 & -0.140451 & -0.161766 \\ 14 & 6 & 0 & -0.323847 & -0.970531 & 0.532523 \\ 15 & 6 & 0 & -0.161477 & -0.900016 & 2.051324 \\ 16 & 1 & 0 & -2.911452 & 1.211101 & -0.311434 \\ 17 & 1 & 0 & -2.343859 & -2.560844 & 0.079597 \\ 18 & 1 & 0 & -4.748254 & -1.755366 & 0.246338 \\ 19 & 1 & 0 & -4.345741 & -0.306138 & -0.688346 \\ 20 & 1 & 0 & -4.281362 & -1.902965 & -1.441543 \\ 21 & 1 & 0 & 1.056524 & 1.599567 & -1.079277 \\ 22 & 1 & 0 & 0.843640 & 1.778608 & 1.976138 \\ 23 & 1 & 0 & 1.433527 & 3.016899 & 0.856066 \\ 24 & 1 & 0 & 2.341941 & 1.510659 & 1.062134 \\ 25 & 1 & 0 & -2.185774 & 3.411795 & -0.622353 \\ 26 & 1 & 0 & -0.555285 & 3.591588 & -1.295662 \\ 27 & 1 & 0 & -0.820341 & 3.801878 & 0.432222 \\ 28 & 1 & 0 & -0.067772 & -1.969719 & 0.182237 \\ 29 & 1 & 0 & 0.885720 & -1.046270 & 2.324037 \\ 30 & 1 & 0 & -0.758784 & -1.696862 & 2.504130 \\ 31 & 1 & 0 & -0.516401 & 0.051037 & 2.455758 \\ ------------------------------------------------------\end{array}$

\section{cTS $_{\text {BN }}$}

$\mathrm{E}(\mathrm{RB}+\mathrm{HF}-\mathrm{LYP})=-829.745905007$ A.U.

Zero-point correction= 0.275739 (Hartree/Particle)

Imaginary Frequency -- $-260.0988 \mathrm{~cm}-1$

\begin{tabular}{|c|c|c|c|c|c|}
\hline \multirow{2}{*}{$\begin{array}{l}\text { Center } \\
\text { Number }\end{array}$} & \multirow{2}{*}{$\begin{array}{l}\text { Atomic } \\
\text { Number }\end{array}$} & \multirow{2}{*}{$\begin{array}{c}\text { Atomic } \\
\text { Type }\end{array}$} & \multicolumn{3}{|c|}{ Coordinates (Angstroms) } \\
\hline & & & $\mathrm{X}$ & $\mathrm{Y}$ & $\mathrm{Z}$ \\
\hline 1 & 9 & 0 & 1.957395 & -0.604545 & -1.186404 \\
\hline 2 & 5 & 0 & 2.426952 & 0.002465 & -0.028958 \\
\hline 3 & 9 & 0 & 2.813066 & 1.307201 & -0.221148 \\
\hline 4 & 9 & 0 & 3.348312 & -0.772270 & 0.631016 \\
\hline 5 & 8 & 0 & 1.185044 & 0.108836 & 0.962175 \\
\hline 6 & 6 & 0 & 0.536894 & -0.955793 & 1.254596 \\
\hline 7 & 6 & 0 & -0.136622 & -0.999393 & 2.596703 \\
\hline 8 & 1 & 0 & 0.914333 & -1.902035 & 0.859567 \\
\hline 9 & 1 & 0 & 0.644493 & -1.174491 & 3.347892 \\
\hline 10 & 1 & 0 & -0.866611 & -1.808070 & 2.664221 \\
\hline 11 & 1 & 0 & -0.607305 & -0.041700 & 2.829289 \\
\hline 12 & 6 & 0 & -1.866538 & -0.116089 & 0.146412 \\
\hline 13 & 6 & 0 & -1.045452 & -1.159424 & -0.188623 \\
\hline 14 & 6 & 0 & -1.527076 & 1.272794 & -0.127243 \\
\hline 15 & 6 & 0 & -0.779806 & -2.161605 & -1.005701 \\
\hline 16 & 6 & 0 & -1.542476 & -2.457623 & -2.273032 \\
\hline 17 & 6 & 0 & -0.424397 & 1.534359 & -0.888699 \\
\hline 18 & 6 & 0 & 0.169522 & 2.852093 & -1.245175 \\
\hline 19 & 6 & 0 & -2.398368 & 2.355082 & 0.472219 \\
\hline 20 & 6 & 0 & -3.096518 & -0.422648 & 0.971929 \\
\hline 21 & 1 & 0 & 0.068885 & -2.813954 & -0.807120 \\
\hline 22 & 1 & 0 & -1.939771 & -3.479861 & -2.255175 \\
\hline 23 & 1 & 0 & -2.373771 & -1.764103 & -2.419373 \\
\hline 24 & 1 & 0 & -0.876723 & -2.388966 & -3.141414 \\
\hline 25 & 1 & 0 & 0.114848 & 0.682793 & -1.292908 \\
\hline 26 & 1 & 0 & 1.220333 & 2.837652 & -0.933426 \\
\hline 27 & 1 & 0 & -0.331030 & 3.713403 & -0.800467 \\
\hline 28 & 1 & 0 & 0.176120 & 2.976070 & -2.336310 \\
\hline 29 & 1 & 0 & -3.440801 & 2.260734 & 0.147086 \\
\hline 30 & 1 & 0 & -2.058824 & 3.349360 & 0.183475 \\
\hline 31 & 1 & 0 & -2.401445 & 2.315800 & 1.567686 \\
\hline
\end{tabular}




\begin{tabular}{|c|c|c|c|c|c|}
\hline 32 & 1 & 0 & -3.035719 & 0.022227 & 1.972609 \\
\hline 33 & 1 & 0 & -3.984239 & 0.006269 & 0.492733 \\
\hline 34 & 1 & 0 & -3.241816 & -1.498134 & 1.077519 \\
\hline
\end{tabular}

\section{cTS $_{\text {BX }}$}

$\mathrm{E}(\mathrm{RB}+\mathrm{HF}-\mathrm{LYP})=-829.745548883 \quad \mathrm{~A} . \mathrm{U}$.

Zero-point correction $=0.276185$ (Hartree/Particle)

Imaginary Frequency -- $-246.6488 \quad \mathrm{~cm}-1$

\begin{tabular}{|c|c|c|c|c|c|}
\hline \multirow{2}{*}{$\begin{array}{l}\text { Center } \\
\text { Number }\end{array}$} & \multirow{2}{*}{$\begin{array}{l}\text { Atomic } \\
\text { Number }\end{array}$} & \multirow{2}{*}{$\begin{array}{c}\text { Atomic } \\
\text { Type }\end{array}$} & \multicolumn{3}{|c|}{ Coordinates (Angstroms) } \\
\hline & & & $\mathrm{X}$ & $\mathrm{Y}$ & Z \\
\hline 1 & 9 & 0 & 2.652648 & 0.426836 & -0.638151 \\
\hline 2 & 5 & 0 & 2.172534 & -0.608388 & 0.128605 \\
\hline 3 & 9 & 0 & 1.730979 & -0.184374 & 1.387962 \\
\hline 4 & 9 & 0 & 3.027721 & -1.676114 & 0.220965 \\
\hline 5 & 8 & 0 & 0.906214 & -1.157261 & -0.636770 \\
\hline 6 & 6 & 0 & -0.001581 & -1.780544 & 0.024786 \\
\hline 7 & 6 & 0 & -0.641016 & -2.958854 & -0.650420 \\
\hline 8 & 1 & 0 & 0.116593 & -1.834136 & 1.108664 \\
\hline 9 & 1 & 0 & -1.494666 & -3.344525 & -0.092346 \\
\hline 10 & 1 & 0 & 0.128608 & -3.740641 & -0.683948 \\
\hline 11 & 1 & 0 & -0.924848 & -2.728614 & -1.679353 \\
\hline 12 & 6 & 0 & -1.134199 & 0.618290 & 0.809192 \\
\hline 13 & 6 & 0 & -1.723485 & -0.439779 & 0.160624 \\
\hline 14 & 6 & 0 & -0.334779 & 1.613103 & 0.113409 \\
\hline 15 & 6 & 0 & -2.846703 & -0.870864 & -0.387166 \\
\hline 16 & 6 & 0 & -4.115200 & -0.055782 & -0.486885 \\
\hline 17 & 6 & 0 & -0.182820 & 1.493250 & -1.237135 \\
\hline 18 & 6 & 0 & 0.666288 & 2.324637 & -2.135681 \\
\hline 19 & 6 & 0 & 0.381358 & 2.662696 & 0.930014 \\
\hline 20 & 6 & 0 & -1.170386 & 0.623615 & 2.316957 \\
\hline 21 & 1 & 0 & -2.905662 & -1.870975 & -0.809808 \\
\hline 22 & 1 & 0 & -4.934412 & -0.547422 & 0.052220 \\
\hline 23 & 1 & 0 & -3.985069 & 0.948207 & -0.076746 \\
\hline 24 & 1 & 0 & -4.435061 & 0.036403 & -1.531832 \\
\hline 25 & 1 & 0 & -0.700063 & 0.669274 & -1.719546 \\
\hline 26 & 1 & 0 & 1.626695 & 1.807660 & -2.257635 \\
\hline 27 & 1 & 0 & 0.875094 & 3.322024 & -1.743502 \\
\hline 28 & 1 & 0 & 0.209330 & 2.420922 & -3.125552 \\
\hline 29 & 1 & 0 & -0.293199 & 3.175785 & 1.622857 \\
\hline 30 & 1 & 0 & 0.842529 & 3.420563 & 0.297055 \\
\hline 31 & 1 & 0 & 1.176485 & 2.189084 & 1.513455 \\
\hline 32 & 1 & 0 & -0.162645 & 0.435984 & 2.703881 \\
\hline 33 & 1 & 0 & -1.850832 & -0.138184 & 2.699729 \\
\hline 34 & 1 & 0 & -1.488249 & 1.602877 & 2.690389 \\
\hline
\end{tabular}

$\mathbf{c P}_{\mathbf{B N}}$

$\mathrm{E}(\mathrm{RB}+\mathrm{HF}-\mathrm{LYP})=-829.803716002 \quad$ A.U.

Zero-point correction $=0.282331$ (Hartree/Particle)

\begin{tabular}{|c|c|c|c|c|c|}
\hline \multirow{2}{*}{$\begin{array}{l}\text { Center } \\
\text { Number }\end{array}$} & \multirow{2}{*}{$\begin{array}{l}\text { Atomic } \\
\text { Number }\end{array}$} & \multirow{2}{*}{$\begin{array}{c}\text { Atomic } \\
\text { Type }\end{array}$} & \multicolumn{3}{|c|}{ Coordinates (Angstroms) } \\
\hline & & & $\mathrm{X}$ & $\mathrm{Y}$ & $\mathrm{Z}$ \\
\hline 1 & 9 & 0 & 2.870185 & 0.161660 & -1.435942 \\
\hline 2 & 5 & 0 & 2.458736 & -0.672118 & -0.439073 \\
\hline 3 & 9 & 0 & 3.113161 & -0.507072 & 0.751951 \\
\hline 4 & 9 & 0 & 2.258094 & -1.971152 & -0.795641 \\
\hline 5 & 6 & 0 & -1.834889 & 0.559185 & -0.035019 \\
\hline
\end{tabular}




$\begin{array}{rrrrrr}6 & 6 & 0 & -1.379389 & -0.835808 & 0.158408 \\ 7 & 6 & 0 & -0.920443 & 1.531692 & -0.257942 \\ 8 & 6 & 0 & -2.053741 & -1.973855 & -0.097872 \\ 9 & 6 & 0 & -3.418612 & -2.188200 & -0.687165 \\ 10 & 6 & 0 & 0.569153 & 1.282881 & -0.332149 \\ 11 & 6 & 0 & 1.391184 & 2.163977 & 0.620222 \\ 12 & 6 & 0 & -1.293272 & 2.977536 & -0.498781 \\ 13 & 6 & 0 & -3.312854 & 0.887429 & 0.080113 \\ 14 & 8 & 0 & 0.895489 & -0.128401 & -0.140655 \\ 15 & 6 & 0 & 0.024461 & -0.961133 & 0.703540 \\ 16 & 6 & 0 & -1.524577 & -0.623495 & 2.184369 \\ 17 & 1 & 0 & -4.137717 & -2.517846 & 0.127274 \\ 18 & 1 & 0 & -3.827456 & -1.305362 & -1.177202 \\ 19 & 1 & 0 & -3.370266 & -2.990826 & -1.431771 \\ 20 & 1 & 0 & 0.911295 & 1.472823 & -1.354351 \\ 21 & 1 & 0 & 2.445049 & 1.892224 & 0.561069 \\ 22 & 1 & 0 & 1.058037 & 2.061991 & 1.655371 \\ 23 & 1 & 0 & 1.291823 & 3.212942 & 0.329667 \\ 24 & 1 & 0 & -2.304710 & 3.078118 & -0.894538 \\ 25 & 1 & 0 & -0.613444 & 3.440253 & -1.222385 \\ 26 & 1 & 0 & -1.233374 & 3.576124 & 0.419567 \\ 27 & 1 & 0 & -3.468182 & 1.855150 & 0.562925 \\ 28 & 1 & 0 & -3.812438 & 0.928312 & -0.895728 \\ 29 & 1 & 0 & -3.829621 & 0.138447 & 0.682555 \\ 30 & 1 & 0 & 0.412342 & -1.960087 & 0.510988 \\ 31 & 1 & 0 & 1.241208 & -0.604242 & 2.449723 \\ 32 & 1 & 0 & -0.315067 & -1.398352 & 2.775828 \\ 33 & 1 & 0 & -0.275631 & 0.335883 & 2.436809 \\ 34 & 1 & & & & \\ -------------------------------------------145\end{array}$

\section{$\mathbf{c P}_{\text {BX }}$}

$\mathrm{E}(\mathrm{RB}+\mathrm{HF}-\mathrm{LYP})=-829.799798961$

A. U.

Zero-point correction $=0.282200$ (Hartree/Particle)

\begin{tabular}{|c|c|c|c|c|c|}
\hline \multirow{2}{*}{$\begin{array}{l}\text { Center } \\
\text { Number }\end{array}$} & \multirow{2}{*}{$\begin{array}{l}\text { Atomic } \\
\text { Number }\end{array}$} & \multirow{2}{*}{$\begin{array}{l}\text { Atomic } \\
\text { Type }\end{array}$} & \multicolumn{3}{|c|}{ Coordinates (Angstroms) } \\
\hline & & & $\mathrm{X}$ & $\mathrm{Y}$ & Z \\
\hline 1 & 9 & 0 & 1.481463 & -1.484467 & -1.277139 \\
\hline 2 & 5 & 0 & 2.152525 & -0.425482 & -0.752979 \\
\hline 3 & 9 & 0 & 2.388071 & 0.602229 & -1.614672 \\
\hline 4 & 9 & 0 & 3.188636 & -0.739475 & 0.065630 \\
\hline 5 & 6 & 0 & -1.784397 & 0.426190 & -0.000665 \\
\hline 6 & 6 & 0 & -1.118006 & -0.828996 & 0.426871 \\
\hline 7 & 6 & 0 & -1.052111 & 1.556922 & -0.128500 \\
\hline 8 & 6 & 0 & -1.515949 & -2.091559 & 0.184630 \\
\hline 9 & 6 & 0 & -2.651809 & -2.603150 & -0.654503 \\
\hline 10 & 6 & 0 & 0.446920 & 1.602007 & 0.075268 \\
\hline 11 & 6 & 0 & 0.906536 & 2.566285 & 1.175417 \\
\hline 12 & 6 & 0 & -1.642676 & 2.890548 & -0.529233 \\
\hline 13 & 6 & 0 & -3.289627 & 0.450793 & -0.195693 \\
\hline 14 & 8 & 0 & 1.011243 & 0.284505 & 0.352348 \\
\hline 15 & 6 & 0 & 0.149599 & -0.555769 & 1.212419 \\
\hline 16 & 6 & 0 & 0.958490 & -1.727333 & 1.741041 \\
\hline 17 & 1 & 0 & -0.910061 & -2.883555 & 0.616492 \\
\hline 18 & 1 & 0 & -3.457087 & -3.018349 & -0.033224 \\
\hline 19 & 1 & 0 & -3.083937 & -1.852170 & -1.314320 \\
\hline 20 & 1 & 0 & -2.290577 & -3.426971 & -1.280724 \\
\hline 21 & 1 & 0 & 0.919239 & 1.892040 & -0.864422 \\
\hline 22 & 1 & 0 & 0.497275 & 2.289803 & 2.151748 \\
\hline 23 & 1 & 0 & 0.580082 & 3.585284 & 0.951522 \\
\hline
\end{tabular}




\begin{tabular}{|c|c|c|c|c|c|}
\hline 24 & 1 & 0 & 1.997427 & 2.560552 & 1.235735 \\
\hline 25 & 1 & 0 & -2.520306 & 2.774112 & -1.167514 \\
\hline 26 & 1 & 0 & -0.914205 & 3.489969 & -1.085556 \\
\hline 27 & 1 & 0 & -1.945349 & 3.485843 & 0.342786 \\
\hline 28 & 1 & 0 & -3.718580 & 1.392778 & 0.154782 \\
\hline 29 & 1 & 0 & -3.768650 & -0.352675 & 0.366414 \\
\hline 30 & 1 & 0 & -3.579110 & 0.335758 & -1.247694 \\
\hline 31 & 1 & 0 & -0.097982 & 0.096867 & 2.057693 \\
\hline 32 & 1 & 0 & 0.365691 & -2.254100 & 2.494092 \\
\hline 33 & 1 & 0 & 1.871841 & -1.360042 & 2.211088 \\
\hline 34 & 1 & 0 & 1.236366 & -2.423985 & 0.950532 \\
\hline
\end{tabular}

\section{cTS $_{\mathrm{CN}}$}

$\mathrm{E}(\mathrm{RB}+\mathrm{HF}-\mathrm{LYP})=-869.061038295 \mathrm{~A} . \mathrm{U}$.

Zero-point correction= 0.304971 (Hartree/Particle)

Imaginary Frequency -- $-255.4784 \quad \mathrm{~cm}-1$

\begin{tabular}{|c|c|c|c|c|c|}
\hline \multirow{2}{*}{$\begin{array}{l}\text { Center } \\
\text { Number }\end{array}$} & \multirow{2}{*}{$\begin{array}{l}\text { Atomic } \\
\text { Number }\end{array}$} & \multirow{2}{*}{$\begin{array}{c}\text { Atomic } \\
\text { Type }\end{array}$} & \multicolumn{3}{|c|}{ Coordinates (Angstroms) } \\
\hline & & & $\mathrm{X}$ & $\mathrm{Y}$ & $\mathrm{Z}$ \\
\hline 1 & 6 & 0 & -1.346083 & -2.413314 & -2.210611 \\
\hline 2 & 6 & 0 & -0.602900 & -2.120277 & -0.930882 \\
\hline 3 & 6 & 0 & -0.835139 & -1.084780 & -0.145532 \\
\hline 4 & 6 & 0 & -1.602476 & 0.007002 & 0.160821 \\
\hline 5 & 6 & 0 & -2.881613 & -0.237684 & 0.947359 \\
\hline 6 & 6 & 0 & -4.115470 & -0.256777 & 0.021757 \\
\hline 7 & 6 & 0 & -1.180180 & 1.368146 & -0.140193 \\
\hline 8 & 6 & 0 & -1.943806 & 2.523697 & 0.473207 \\
\hline 9 & 6 & 0 & -0.079766 & 1.543816 & -0.929834 \\
\hline 10 & 6 & 0 & 0.598517 & 2.812155 & -1.314528 \\
\hline 11 & 1 & 0 & -1.815610 & -3.403777 & -2.168439 \\
\hline 12 & 1 & 0 & -2.120199 & -1.669420 & -2.412605 \\
\hline 13 & 1 & 0 & -0.649111 & -2.425755 & -3.056737 \\
\hline 14 & 1 & 0 & 0.210016 & -2.805746 & -0.698065 \\
\hline 15 & 1 & 0 & -3.014492 & 0.527167 & 1.719466 \\
\hline 16 & 1 & 0 & -2.810743 & -1.201594 & 1.456981 \\
\hline 17 & 1 & 0 & -5.024347 & -0.414260 & 0.610269 \\
\hline 18 & 1 & 0 & -4.227161 & 0.681727 & -0.528663 \\
\hline 19 & 1 & 0 & -4.040030 & -1.067023 & -0.708933 \\
\hline 20 & 1 & 0 & -3.015993 & 2.465498 & 0.261213 \\
\hline 21 & 1 & 0 & -1.592390 & 3.481505 & 0.090122 \\
\hline 22 & 1 & 0 & -1.831654 & 2.549033 & 1.563631 \\
\hline 23 & 1 & 0 & 0.388787 & 0.652594 & -1.336569 \\
\hline 24 & 1 & 0 & 1.650564 & 2.728813 & -1.017537 \\
\hline 25 & 1 & 0 & 0.167247 & 3.712075 & -0.873906 \\
\hline 26 & 1 & 0 & 0.596891 & 2.921020 & -2.407284 \\
\hline 27 & 6 & 0 & 0.749661 & -0.913508 & 1.293697 \\
\hline 28 & 6 & 0 & 0.082464 & -0.854799 & 2.639365 \\
\hline 29 & 8 & 0 & 1.456072 & 0.098210 & 0.950762 \\
\hline 30 & 5 & 0 & 2.687782 & -0.123567 & -0.032912 \\
\hline 31 & 9 & 0 & 3.555462 & -0.935345 & 0.655470 \\
\hline 32 & 9 & 0 & 2.181572 & -0.735588 & -1.171775 \\
\hline 33 & 9 & 0 & 3.160006 & 1.145780 & -0.265973 \\
\hline 34 & 1 & 0 & 1.075433 & -1.897365 & 0.947311 \\
\hline 35 & 1 & 0 & 0.859047 & -1.028364 & 3.395632 \\
\hline 36 & 1 & 0 & -0.683930 & -1.623720 & 2.752084 \\
\hline 37 & 1 & 0 & -0.342424 & 0.134063 & 2.823240 \\
\hline
\end{tabular}


cTS $_{\mathbf{C X}}$

$\mathrm{E}(\mathrm{RB}+\mathrm{HF}-\mathrm{LYP})=-869.059235153 \quad \mathrm{~A} . \mathrm{U}$.

Zero-point correction $=0.305004$ (Hartree/Particle)

Imaginary Frequency -- $\quad-246.5939 \mathrm{~cm}-1$

\begin{tabular}{|c|c|c|c|c|c|}
\hline \multirow{2}{*}{$\begin{array}{l}\text { Center } \\
\text { Number }\end{array}$} & \multirow{2}{*}{$\begin{array}{l}\text { Atomic } \\
\text { Number }\end{array}$} & \multirow{2}{*}{$\begin{array}{l}\text { Atomic } \\
\text { Type }\end{array}$} & \multicolumn{3}{|c|}{ Coordinates (Angstroms) } \\
\hline & & & $\mathrm{x}$ & $\mathrm{Y}$ & $\mathrm{Z}$ \\
\hline 1 & 9 & 0 & 2.762269 & -0.762064 & 0.414445 \\
\hline 2 & 5 & 0 & 2.352876 & 0.207387 & -0.472758 \\
\hline 3 & 9 & 0 & 1.601448 & -0.319758 & -1.529221 \\
\hline 4 & 9 & 0 & 3.366949 & 1.015524 & -0.917591 \\
\hline 5 & 8 & 0 & 1.387316 & 1.156186 & 0.337697 \\
\hline 6 & 6 & 0 & 0.484609 & 1.809696 & -0.303126 \\
\hline 7 & 6 & 0 & 0.186512 & 3.198464 & 0.183625 \\
\hline 8 & 1 & 0 & 0.407198 & 1.631339 & -1.376875 \\
\hline 9 & 1 & 0 & -0.675452 & 3.638975 & -0.318299 \\
\hline 10 & 1 & 0 & 1.070455 & 3.800412 & -0.063183 \\
\hline 11 & 1 & 0 & 0.057995 & 3.228637 & 1.267684 \\
\hline 12 & 6 & 0 & -1.126682 & -0.430853 & -0.351238 \\
\hline 13 & 6 & 0 & -1.425706 & 0.831742 & 0.100847 \\
\hline 14 & 6 & 0 & -0.401237 & -1.391186 & 0.462874 \\
\hline 15 & 6 & 0 & -2.352107 & 1.582153 & 0.672735 \\
\hline 16 & 6 & 0 & -3.703446 & 1.079930 & 1.126878 \\
\hline 17 & 6 & 0 & -0.016881 & -1.015217 & 1.717498 \\
\hline 18 & 6 & 0 & 0.832033 & -1.783604 & 2.671352 \\
\hline 19 & 6 & 0 & 0.020407 & -2.708845 & -0.145792 \\
\hline 20 & 6 & 0 & -1.460012 & -0.748563 & -1.797745 \\
\hline 21 & 6 & 0 & -2.812518 & -1.477287 & -1.927360 \\
\hline 22 & 1 & 0 & -2.168992 & 2.638033 & 0.856650 \\
\hline 23 & 1 & 0 & -3.811377 & 0.004539 & 0.969042 \\
\hline 24 & 1 & 0 & -3.853802 & 1.289111 & 2.192960 \\
\hline 25 & 1 & 0 & -4.509889 & 1.592624 & 0.588042 \\
\hline 26 & 1 & 0 & -0.313344 & -0.025072 & 2.050135 \\
\hline 27 & 1 & 0 & 0.765071 & -2.866201 & 2.541915 \\
\hline 28 & 1 & 0 & 1.876313 & -1.495299 & 2.493734 \\
\hline 29 & 1 & 0 & 0.584948 & -1.533635 & 3.707297 \\
\hline 30 & 1 & 0 & 0.890667 & -2.546582 & -0.788598 \\
\hline 31 & 1 & 0 & -0.771058 & -3.163098 & -0.746864 \\
\hline 32 & 1 & 0 & 0.302897 & -3.430538 & 0.621597 \\
\hline 33 & 1 & 0 & -1.512145 & 0.188281 & -2.359191 \\
\hline 34 & 1 & 0 & -0.657561 & -1.340742 & -2.242206 \\
\hline 35 & 1 & 0 & -3.015365 & -1.701404 & -2.978882 \\
\hline 36 & 1 & 0 & -2.824917 & -2.421266 & -1.374620 \\
\hline 37 & 1 & 0 & -3.630389 & -0.856441 & -1.550110 \\
\hline
\end{tabular}

\section{$\mathbf{c P}_{\mathrm{CN}}$}

$\mathrm{E}(\mathrm{RB}+\mathrm{HF}-\mathrm{LYP})=-869.116661685 \quad \mathrm{~A} . \mathrm{U}$.

Zero-point correction $=0.311480$ (Hartree/Particle)

\begin{tabular}{|c|c|c|c|c|c|}
\hline \multirow{2}{*}{$\begin{array}{l}\text { Center } \\
\text { Number }\end{array}$} & \multirow{2}{*}{$\begin{array}{l}\text { Atomic } \\
\text { Number }\end{array}$} & \multirow{2}{*}{$\begin{array}{l}\text { Atomic } \\
\text { Type }\end{array}$} & \multicolumn{3}{|c|}{ Coordinates (Angstroms) } \\
\hline & & & $\mathrm{X}$ & $\mathrm{Y}$ & Z \\
\hline 1 & 9 & 0 & 2.963277 & 0.451215 & -1.582328 \\
\hline 2 & 5 & 0 & 2.700587 & -0.489444 & -0.631862 \\
\hline 3 & 9 & 0 & 3.450274 & -0.376240 & 0.508095 \\
\hline 4 & 9 & 0 & 2.549608 & -1.765467 & -1.082364 \\
\hline 5 & 6 & 0 & -1.619607 & 0.373540 & 0.161826 \\
\hline 6 & 6 & 0 & -1.054225 & -0.992246 & 0.266672 \\
\hline 7 & 6 & 0 & -0.784107 & 1.432766 & 0.013704 \\
\hline
\end{tabular}




\begin{tabular}{|c|c|c|c|c|c|}
\hline 8 & 6 & 0 & -1.649499 & -2.191445 & 0.092619 \\
\hline 9 & 6 & 0 & -3.044604 & -2.593316 & -0.291311 \\
\hline 10 & 6 & 0 & 0.712774 & 1.308752 & -0.169768 \\
\hline 11 & 6 & 0 & 1.550286 & 2.150733 & 0.804606 \\
\hline 12 & 6 & 0 & -1.243953 & 2.872107 & -0.036310 \\
\hline 13 & 6 & 0 & -3.126099 & 0.584180 & 0.275498 \\
\hline 14 & 6 & 0 & -3.839121 & 0.813984 & -1.070361 \\
\hline 15 & 8 & 0 & 1.139552 & -0.084785 & -0.152145 \\
\hline 16 & 6 & 0 & 0.401373 & -1.036049 & 0.685289 \\
\hline 17 & 6 & 0 & 0.651511 & -0.799251 & 2.174236 \\
\hline 18 & 1 & 0 & -0.999814 & -3.052105 & 0.254849 \\
\hline 19 & 1 & 0 & -3.586403 & -2.996905 & 0.575185 \\
\hline 20 & 1 & 0 & -3.645368 & -1.794529 & -0.719968 \\
\hline 21 & 1 & 0 & -2.997120 & -3.404897 & -1.026564 \\
\hline 22 & 1 & 0 & 0.963432 & 1.623994 & -1.187817 \\
\hline 23 & 1 & 0 & 2.608386 & 1.929114 & 0.666296 \\
\hline 24 & 1 & 0 & 1.284157 & 1.957239 & 1.845978 \\
\hline 25 & 1 & 0 & 1.395254 & 3.213225 & 0.601380 \\
\hline 26 & 1 & 0 & -2.321312 & 2.970706 & -0.153422 \\
\hline 27 & 1 & 0 & -0.772365 & 3.403017 & -0.872305 \\
\hline 28 & 1 & 0 & -0.959585 & 3.410625 & 0.876098 \\
\hline 29 & 1 & 0 & -3.322825 & 1.441120 & 0.928268 \\
\hline 30 & 1 & 0 & -3.570720 & -0.271523 & 0.787609 \\
\hline 31 & 1 & 0 & -4.921201 & 0.901844 & -0.926798 \\
\hline 32 & 1 & 0 & -3.489252 & 1.730062 & -1.554640 \\
\hline 33 & 1 & 0 & -3.655767 & -0.004151 & -1.772801 \\
\hline 34 & 1 & 0 & 0.843355 & -1.985774 & 0.389022 \\
\hline 35 & 1 & 0 & 1.723693 & -0.707078 & 2.360728 \\
\hline 36 & 1 & 0 & 0.270003 & -1.658670 & 2.733553 \\
\hline 37 & 1 & 0 & 0.137302 & 0.092089 & 2.540994 \\
\hline
\end{tabular}

$\mathbf{c P}_{\mathrm{CX}}$

$\mathrm{E}(\mathrm{RB}+\mathrm{HF}-\mathrm{LYP})=-869.119056733 \quad \mathrm{~A} \cdot \mathrm{U}$.

Zero-point correction $=0.310457$ (Hartree/Particle)

\begin{tabular}{|c|c|c|c|c|c|}
\hline \multirow{2}{*}{$\begin{array}{l}\text { Center } \\
\text { Number }\end{array}$} & \multirow{2}{*}{$\begin{array}{l}\text { Atomic } \\
\text { Number }\end{array}$} & \multirow{2}{*}{$\begin{array}{c}\text { Atomic } \\
\text { Type }\end{array}$} & \multicolumn{3}{|c|}{ Coordinates (Angstroms) } \\
\hline & & & $\mathrm{X}$ & $\mathrm{Y}$ & $\mathrm{Z}$ \\
\hline 1 & 9 & 0 & -3.420530 & 0.200976 & -0.896851 \\
\hline 2 & 5 & 0 & -2.793675 & -0.215617 & 0.240736 \\
\hline 3 & 9 & 0 & -3.002612 & -1.519451 & 0.582703 \\
\hline 4 & 9 & 0 & -2.866673 & 0.673931 & 1.276413 \\
\hline 5 & 6 & 0 & 1.644693 & 0.383268 & -0.097027 \\
\hline 6 & 6 & 0 & 1.108662 & -1.001627 & -0.157661 \\
\hline 7 & 6 & 0 & 0.755249 & 1.376641 & -0.309066 \\
\hline 8 & 6 & 0 & 1.676871 & -2.079440 & -0.727818 \\
\hline 9 & 6 & 0 & 2.963152 & -2.166601 & -1.498000 \\
\hline 10 & 6 & 0 & -0.661639 & 1.064630 & -0.741967 \\
\hline 11 & 6 & 0 & -0.805621 & 1.000532 & -2.264066 \\
\hline 12 & 6 & 0 & 1.044685 & 2.851849 & -0.208007 \\
\hline 13 & 6 & 0 & 3.078703 & 0.620277 & 0.348135 \\
\hline 14 & 6 & 0 & 3.214920 & 0.784403 & 1.872891 \\
\hline 15 & 8 & 0 & -1.171525 & -0.209487 & -0.194227 \\
\hline 16 & 6 & 0 & -0.251625 & -1.146099 & 0.487219 \\
\hline 17 & 6 & 0 & -0.268778 & -0.914650 & 1.996395 \\
\hline 18 & 1 & 0 & 1.125561 & -3.016845 & -0.656024 \\
\hline 19 & 1 & 0 & 3.761893 & -2.632419 & -0.905300 \\
\hline 20 & 1 & 0 & 2.822177 & -2.801857 & -2.379559 \\
\hline 21 & 1 & 0 & 3.317593 & -1.193340 & -1.842755 \\
\hline 22 & 1 & 0 & -1.336722 & 1.820792 & -0.332089 \\
\hline
\end{tabular}




\begin{tabular}{|c|c|c|c|c|c|}
\hline 23 & 1 & 0 & -0.506770 & 1.955220 & -2.708536 \\
\hline 24 & 1 & 0 & -0.164592 & 0.213618 & -2.669561 \\
\hline 25 & 1 & 0 & -1.843742 & 0.797922 & -2.528247 \\
\hline 26 & 1 & 0 & 0.746830 & 3.379187 & -1.122572 \\
\hline 27 & 1 & 0 & 2.099352 & 3.063614 & -0.029510 \\
\hline 28 & 1 & 0 & 0.468040 & 3.300606 & 0.611160 \\
\hline 29 & 1 & 0 & 3.703043 & -0.219645 & 0.036931 \\
\hline 30 & 1 & 0 & 3.489508 & 1.504816 & -0.149975 \\
\hline 31 & 1 & 0 & 4.263390 & 0.927268 & 2.154394 \\
\hline 32 & 1 & 0 & 2.647060 & 1.648049 & 2.232578 \\
\hline 33 & 1 & 0 & 2.843965 & -0.100725 & 2.398752 \\
\hline 34 & 1 & 0 & -0.680839 & -2.121838 & 0.259514 \\
\hline 35 & 1 & 0 & 0.394579 & -1.644959 & 2.470870 \\
\hline 36 & 1 & 0 & -1.275541 & -1.051815 & 2.391253 \\
\hline 37 & 1 & 0 & 0.078486 & 0.088118 & 2.252252 \\
\hline
\end{tabular}

\section{cTS' $_{\text {CN }}$}

$\mathrm{E}(\mathrm{RB}+\mathrm{HF}-\mathrm{LYP})=-869.054308241$ A.U.

Zero-point correction $=0.303958$ (Hartree/Particle)

Imaginary Frequency -- $-225.8004 \mathrm{~cm}-1$

\begin{tabular}{|c|c|c|c|c|c|}
\hline \multirow{2}{*}{$\begin{array}{l}\text { Center } \\
\text { Number }\end{array}$} & \multirow{2}{*}{$\begin{array}{l}\text { Atomic } \\
\text { Number }\end{array}$} & \multirow{2}{*}{$\begin{array}{c}\text { Atomic } \\
\text { Type }\end{array}$} & \multicolumn{3}{|c|}{ Coordinates (Angstroms) } \\
\hline & & & $\mathrm{X}$ & $\mathrm{Y}$ & $\mathrm{Z}$ \\
\hline 1 & 6 & 0 & 0.224195 & -2.322241 & -2.357837 \\
\hline 2 & 6 & 0 & 0.227777 & -2.008571 & -0.880107 \\
\hline 3 & 6 & 0 & -0.563432 & -1.110427 & -0.307071 \\
\hline 4 & 6 & 0 & -1.680343 & -0.325808 & -0.360914 \\
\hline 5 & 6 & 0 & -3.037162 & -1.025002 & -0.245168 \\
\hline 6 & 6 & 0 & -3.472517 & -1.403281 & 1.188042 \\
\hline 7 & 6 & 0 & -1.622278 & 1.139488 & -0.475010 \\
\hline 8 & 6 & 0 & -2.870360 & 1.939853 & -0.143608 \\
\hline 9 & 6 & 0 & -0.465232 & 1.716570 & -0.919407 \\
\hline 10 & 6 & 0 & -0.175037 & 3.170862 & -1.109608 \\
\hline 11 & 1 & 0 & 0.232013 & -3.406265 & -2.526397 \\
\hline 12 & 1 & 0 & -0.640798 & -1.890297 & -2.869581 \\
\hline 13 & 1 & 0 & 1.135301 & -1.911686 & -2.812581 \\
\hline 14 & 1 & 0 & 1.025760 & -2.484463 & -0.315225 \\
\hline 15 & 1 & 0 & -2.982906 & -1.945348 & -0.839534 \\
\hline 16 & 1 & 0 & -3.806090 & -0.391849 & -0.699757 \\
\hline 17 & 1 & 0 & -4.450971 & -1.895439 & 1.165127 \\
\hline 18 & 1 & 0 & -2.755966 & -2.098425 & 1.638190 \\
\hline 19 & 1 & 0 & -3.554247 & -0.524583 & 1.836655 \\
\hline 20 & 1 & 0 & -3.614427 & 1.886180 & -0.949850 \\
\hline 21 & 1 & 0 & -2.639100 & 2.995990 & 0.007424 \\
\hline 22 & 1 & 0 & -3.357539 & 1.579574 & 0.768705 \\
\hline 23 & 1 & 0 & 0.359479 & 1.063355 & -1.188606 \\
\hline 24 & 1 & 0 & 0.741983 & 3.431745 & -0.564887 \\
\hline 25 & 1 & 0 & -0.975279 & 3.838540 & -0.782787 \\
\hline 26 & 1 & 0 & 0.032863 & 3.380384 & -2.168550 \\
\hline 27 & 6 & 0 & 0.569871 & -0.439840 & 1.510183 \\
\hline 28 & 6 & 0 & -0.405010 & 0.042213 & 2.533710 \\
\hline 29 & 8 & 0 & 1.384309 & 0.453476 & 1.022808 \\
\hline 30 & 5 & 0 & 2.695840 & 0.070852 & 0.219462 \\
\hline 31 & 9 & 0 & 2.959815 & -1.295602 & 0.520042 \\
\hline 32 & 9 & 0 & 2.390577 & 0.252546 & -1.155534 \\
\hline 33 & 9 & 0 & 3.681251 & 0.942487 & 0.685509 \\
\hline 34 & 1 & 0 & 0.910473 & -1.472648 & 1.547869 \\
\hline 35 & 1 & 0 & 0.157362 & 0.264731 & 3.452012 \\
\hline 36 & 1 & 0 & -1.158892 & -0.711026 & 2.761472 \\
\hline
\end{tabular}




\section{cTS' ${ }_{\mathrm{CX}}$}

$\mathrm{E}(\mathrm{RB}+\mathrm{HF}-\mathrm{LYP})=-869.054483067 \quad \mathrm{~A} . \mathrm{U}$.

Zero-point correction $=0.303906$ (Hartree/Particle)

Imaginary Frequency -- $-175.9674 \quad \mathrm{~cm}-1$

\begin{tabular}{|c|c|c|c|c|c|}
\hline \multirow{2}{*}{$\begin{array}{l}\text { Center } \\
\text { Number }\end{array}$} & \multirow{2}{*}{$\begin{array}{l}\text { Atomic } \\
\text { Number }\end{array}$} & \multirow{2}{*}{$\begin{array}{l}\text { Atomic } \\
\text { Type }\end{array}$} & \multicolumn{3}{|c|}{ Coordinates (Angstroms) } \\
\hline & & & $\mathrm{X}$ & $\mathrm{Y}$ & Z \\
\hline 1 & 9 & 0 & 2.774148 & 0.557948 & -0.593759 \\
\hline 2 & 5 & 0 & 2.299881 & -0.571940 & 0.089125 \\
\hline 3 & 9 & 0 & 1.789736 & -0.258104 & 1.387636 \\
\hline 4 & 9 & 0 & 3.220833 & -1.629852 & 0.137159 \\
\hline 5 & 8 & 0 & 1.071986 & -1.131771 & -0.745219 \\
\hline 6 & 6 & 0 & 0.122022 & -1.820570 & -0.182354 \\
\hline 7 & 6 & 0 & -0.481031 & -2.936654 & -0.967682 \\
\hline 8 & 1 & 0 & 0.111149 & -1.872382 & 0.903347 \\
\hline 9 & 1 & 0 & -1.378518 & -3.334407 & -0.492605 \\
\hline 10 & 1 & 0 & 0.267529 & -3.742315 & -1.003334 \\
\hline 11 & 1 & 0 & -0.691699 & -2.639010 & -1.997229 \\
\hline 12 & 6 & 0 & -1.224742 & 0.663852 & 0.548174 \\
\hline 13 & 6 & 0 & -1.766294 & -0.383211 & -0.145230 \\
\hline 14 & 6 & 0 & -0.260140 & 1.588520 & -0.052881 \\
\hline 15 & 6 & 0 & -2.804688 & -0.881612 & -0.798898 \\
\hline 16 & 6 & 0 & -4.040602 & -0.085443 & -1.169920 \\
\hline 17 & 6 & 0 & -0.033671 & 1.517015 & -1.399763 \\
\hline 18 & 6 & 0 & 0.889891 & 2.363497 & -2.214430 \\
\hline 19 & 6 & 0 & 0.465114 & 2.574050 & 0.839557 \\
\hline 20 & 6 & 0 & -1.630684 & 0.813960 & 2.014934 \\
\hline 21 & 6 & 0 & -1.015798 & -0.234990 & 2.969813 \\
\hline 22 & 1 & 0 & -2.814481 & -1.917209 & -1.130651 \\
\hline 23 & 1 & 0 & -3.975712 & 0.949138 & -0.821713 \\
\hline 24 & 1 & 0 & -4.183390 & -0.072897 & -2.258615 \\
\hline 25 & 1 & 0 & -4.939312 & -0.542488 & -0.734745 \\
\hline 26 & 1 & 0 & -0.571763 & 0.752858 & -1.955534 \\
\hline 27 & 1 & 0 & 1.083024 & 3.343398 & -1.770056 \\
\hline 28 & 1 & 0 & 1.855420 & 1.848048 & -2.304107 \\
\hline 29 & 1 & 0 & 0.492508 & 2.511692 & -3.224496 \\
\hline 30 & 1 & 0 & 0.919471 & 2.053418 & 1.686826 \\
\hline 31 & 1 & 0 & -0.203372 & 3.353274 & 1.228755 \\
\hline 32 & 1 & 0 & 1.277398 & 3.065288 & 0.303448 \\
\hline 33 & 1 & 0 & -1.362024 & 1.814920 & 2.361925 \\
\hline 34 & 1 & 0 & -2.724837 & 0.735605 & 2.065366 \\
\hline 35 & 1 & 0 & -1.315675 & -0.021275 & 4.001492 \\
\hline 36 & 1 & 0 & -1.374580 & -1.240265 & 2.719841 \\
\hline 37 & 1 & 0 & 0.074865 & -0.231938 & 2.910969 \\
\hline
\end{tabular}

D

$\mathrm{E}(\mathrm{RB}+\mathrm{HF}-\mathrm{LYP})=-312.023629055 \quad \mathrm{~A} . \mathrm{U}$.

Zero-point correction $=0.173794$ (Hartree/Particle)

\begin{tabular}{|c|c|c|c|c|c|}
\hline \multirow{2}{*}{$\begin{array}{l}\text { Center } \\
\text { Number }\end{array}$} & \multirow{2}{*}{$\begin{array}{l}\text { Atomic } \\
\text { Number }\end{array}$} & \multirow{2}{*}{$\begin{array}{c}\text { Atomic } \\
\text { Type }\end{array}$} & \multicolumn{3}{|c|}{ Coordinates (Angstroms) } \\
\hline & & & $\mathrm{X}$ & $\mathrm{Y}$ & Z \\
\hline 1 & 6 & 0 & 0.886413 & 0.377883 & -0.109528 \\
\hline 2 & 6 & 0 & 1.653960 & -0.668612 & 0.111557 \\
\hline 3 & 6 & 0 & -0.600586 & 0.302658 & 0.053344 \\
\hline
\end{tabular}




$\begin{array}{rrrrrr}4 & 6 & 0 & 2.430236 & -1.695131 & 0.340133 \\ 5 & 6 & 0 & -1.262605 & -0.811081 & -0.309784 \\ 6 & 6 & 0 & -2.729890 & -1.105315 & -0.197632 \\ 7 & 6 & 0 & -1.259505 & 1.534195 & 0.632904 \\ 8 & 6 & 0 & 1.509843 & 1.698576 & -0.530884 \\ 9 & 1 & 0 & 2.869871 & -1.874805 & 1.319915 \\ 10 & 1 & 0 & 2.668165 & -2.417895 & -0.438782 \\ 11 & 1 & 0 & -0.667558 & -1.615799 & -0.739075 \\ 12 & 1 & 0 & -3.160157 & -1.321456 & -1.184159 \\ 13 & 1 & 0 & -3.307023 & -0.290419 & 0.244293 \\ 14 & 1 & 0 & -2.897295 & -2.001357 & 0.414322 \\ 15 & 1 & 0 & -0.808532 & 1.802841 & 1.596023 \\ 16 & 1 & 0 & -2.329071 & 1.394105 & 0.794098 \\ 17 & 1 & 0 & -1.137420 & 2.402069 & -0.025940 \\ 18 & 1 & 0 & 1.067017 & 2.058349 & -1.467453 \\ 19 & 1 & 0 & 2.585984 & 1.589415 & -0.678620 \\ 20 & 1 & 0 & 1.348832 & 2.475920 & 0.224716 \\ ----------------------------------------------------------\end{array}$

\section{cTS $_{\text {DN }}$}

$\mathrm{E}(\mathrm{RB}+\mathrm{HF}-\mathrm{LYP})=-790.423303628 \quad \mathrm{~A} . \mathrm{U}$. Zero-point correction $=0.247450$ (Hartree/Particle) Imaginary Frequency -- $\quad-289.2717 \quad \mathrm{~cm}-1$

\begin{tabular}{|c|c|c|c|c|c|}
\hline \multirow{2}{*}{$\begin{array}{l}\text { Center } \\
\text { Number }\end{array}$} & \multirow{2}{*}{$\begin{array}{l}\text { Atomic } \\
\text { Number }\end{array}$} & \multirow{2}{*}{$\begin{array}{l}\text { Atomic } \\
\text { Type }\end{array}$} & \multicolumn{3}{|c|}{ Coordinates (Angstroms) } \\
\hline & & & $\mathrm{X}$ & $\mathrm{Y}$ & $\mathrm{Z}$ \\
\hline 1 & 9 & 0 & -2.547358 & 1.416313 & -0.593689 \\
\hline 2 & 5 & 0 & -2.316084 & 0.167019 & -0.072193 \\
\hline 3 & 9 & 0 & -3.274496 & -0.766920 & -0.376981 \\
\hline 4 & 9 & 0 & -2.010929 & 0.187716 & 1.283092 \\
\hline 5 & 8 & 0 & -1.000556 & -0.325222 & -0.829124 \\
\hline 6 & 6 & 0 & -0.513966 & -1.471553 & -0.532342 \\
\hline 7 & 6 & 0 & 0.213048 & -2.214047 & -1.617088 \\
\hline 8 & 1 & 0 & -1.046044 & -2.071056 & 0.210707 \\
\hline 9 & 1 & 0 & 0.793719 & -3.049933 & -1.222861 \\
\hline 10 & 1 & 0 & 0.854759 & -1.542583 & -2.191247 \\
\hline 11 & 1 & 0 & -0.545733 & -2.612569 & -2.303089 \\
\hline 12 & 6 & 0 & 0.163861 & 2.996548 & 0.234999 \\
\hline 13 & 6 & 0 & 0.626881 & 1.609043 & 0.503513 \\
\hline 14 & 6 & 0 & 1.751687 & 0.982404 & 0.046526 \\
\hline 15 & 6 & 0 & 1.926615 & -0.426233 & 0.356930 \\
\hline 16 & 6 & 0 & 0.972476 & -1.166749 & 1.003773 \\
\hline 17 & 6 & 0 & 0.553550 & -1.697005 & 2.134940 \\
\hline 18 & 6 & 0 & 2.792497 & 1.666654 & -0.812926 \\
\hline 19 & 6 & 0 & 3.135692 & -1.158345 & -0.179803 \\
\hline 20 & 1 & 0 & -0.799684 & 2.927018 & -0.285027 \\
\hline 21 & 1 & 0 & 0.853565 & 3.602382 & -0.354580 \\
\hline 22 & 1 & 0 & -0.042990 & 3.512763 & 1.181093 \\
\hline 23 & 1 & 0 & -0.054999 & 1.017847 & 1.106641 \\
\hline 24 & 1 & 0 & -0.355422 & -2.285940 & 2.211836 \\
\hline 25 & 1 & 0 & 1.100873 & -1.531163 & 3.061812 \\
\hline 26 & 1 & 0 & 2.776559 & 1.291079 & -1.843174 \\
\hline 27 & 1 & 0 & 3.805575 & 1.508656 & -0.428951 \\
\hline 28 & 1 & 0 & 2.627922 & 2.743256 & -0.857294 \\
\hline 29 & 1 & 0 & 3.230614 & -1.053627 & -1.266409 \\
\hline 30 & 1 & 0 & 3.102179 & -2.218300 & 0.072467 \\
\hline 31 & 1 & 0 & 4.044972 & -0.729435 & 0.259102 \\
\hline
\end{tabular}




\section{cTS $_{\mathrm{DX}}$}

\begin{tabular}{|c|c|c|c|c|c|}
\hline \multirow{2}{*}{$\begin{array}{l}\text { Center } \\
\text { Number }\end{array}$} & \multirow{2}{*}{$\begin{array}{l}\text { Atomic } \\
\text { Number }\end{array}$} & \multirow{2}{*}{$\begin{array}{l}\text { Atomic } \\
\text { Type }\end{array}$} & \multicolumn{3}{|c|}{ Coordinates (Angstroms) } \\
\hline & & & $\mathrm{X}$ & $\mathrm{Y}$ & Z \\
\hline 1 & 9 & 0 & 2.447929 & 0.036844 & -0.387661 \\
\hline 2 & 5 & 0 & 1.726102 & -0.901492 & 0.311743 \\
\hline 3 & 9 & 0 & 1.219608 & -0.399349 & 1.516492 \\
\hline 4 & 9 & 0 & 2.383632 & -2.090782 & 0.490114 \\
\hline 5 & 8 & 0 & 0.480342 & -1.247620 & -0.594332 \\
\hline 6 & 6 & 0 & -0.586409 & -1.703246 & -0.041080 \\
\hline 7 & 6 & 0 & -1.334415 & -2.768103 & -0.789798 \\
\hline 8 & 1 & 0 & -0.598944 & -1.761071 & 1.049054 \\
\hline 9 & 1 & 0 & -2.297776 & -2.998498 & -0.333885 \\
\hline 10 & 1 & 0 & -0.707870 & -3.667818 & -0.739601 \\
\hline 11 & 1 & 0 & -1.458399 & -2.508684 & -1.843244 \\
\hline 12 & 6 & 0 & -1.380253 & 0.868951 & 0.608421 \\
\hline 13 & 6 & 0 & -2.058341 & -0.098377 & -0.092362 \\
\hline 14 & 6 & 0 & -0.353446 & 1.694620 & -0.000748 \\
\hline 15 & 6 & 0 & -3.169086 & -0.355829 & -0.756897 \\
\hline 16 & 6 & 0 & -0.084359 & 1.519267 & -1.327362 \\
\hline 17 & 6 & 0 & 0.985272 & 2.171550 & -2.132999 \\
\hline 18 & 6 & 0 & 0.441442 & 2.625688 & 0.883428 \\
\hline 19 & 6 & 0 & -1.589839 & 0.922539 & 2.100374 \\
\hline 20 & 1 & 0 & -3.396558 & -1.321571 & -1.194037 \\
\hline 21 & 1 & 0 & -0.684719 & 0.788707 & -1.860920 \\
\hline 22 & 1 & 0 & 1.849679 & 1.495230 & -2.146027 \\
\hline 23 & 1 & 0 & 1.320307 & 3.127654 & -1.726150 \\
\hline 24 & 1 & 0 & 0.659492 & 2.319699 & -3.167154 \\
\hline 25 & 1 & 0 & -0.205071 & 3.277310 & 1.480279 \\
\hline 26 & 1 & 0 & 1.105742 & 3.264081 & 0.301620 \\
\hline 27 & 1 & 0 & 1.061440 & 2.038083 & 1.566759 \\
\hline 28 & 1 & 0 & -0.683335 & 0.572203 & 2.606209 \\
\hline 29 & 1 & 0 & -2.434987 & 0.303952 & 2.405174 \\
\hline 30 & 1 & 0 & -1.769230 & 1.953377 & 2.423321 \\
\hline 31 & 1 & 0 & -3.916932 & 0.422989 & -0.901822 \\
\hline
\end{tabular}

\section{$\mathbf{c P}_{\mathrm{DN}}$}

$\mathrm{E}(\mathrm{RB}+\mathrm{HF}-\mathrm{LYP})=-790.489067312 \quad \mathrm{~A} . \mathrm{U}$.

Zero-point correction $=0.254058$ (Hartree/Particle)

\begin{tabular}{|c|c|c|c|c|c|}
\hline \multirow{2}{*}{$\begin{array}{l}\text { Center } \\
\text { Number }\end{array}$} & \multirow{2}{*}{$\begin{array}{l}\text { Atomic } \\
\text { Number }\end{array}$} & \multirow{2}{*}{$\begin{array}{c}\text { Atomic } \\
\text { Type }\end{array}$} & \multicolumn{3}{|c|}{ Coordinates (Angstroms) } \\
\hline & & & $\mathrm{X}$ & $\mathrm{Y}$ & Z \\
\hline 1 & 9 & 0 & 2.529825 & 1.006782 & -1.286035 \\
\hline 2 & 5 & 0 & 2.368109 & -0.083306 & -0.486386 \\
\hline 3 & 9 & 0 & 2.946219 & 0.022244 & 0.749620 \\
\hline 4 & 9 & 0 & 2.531082 & -1.293530 & -1.086396 \\
\hline 5 & 6 & 0 & -2.101790 & -0.106001 & -0.224986 \\
\hline 6 & 6 & 0 & -1.327645 & -1.357862 & -0.117430 \\
\hline 7 & 6 & 0 & -1.472960 & 1.091924 & -0.173287 \\
\hline 8 & 6 & 0 & -1.826357 & -2.575233 & -0.384569 \\
\hline 9 & 6 & 0 & 0.027689 & 1.232199 & -0.053827 \\
\hline 10 & 6 & 0 & 0.494401 & 1.990942 & 1.197147 \\
\hline 11 & 6 & 0 & -2.163898 & 2.433217 & -0.267497 \\
\hline 12 & 6 & 0 & -3.594242 & -0.253833 & -0.413005 \\
\hline 13 & 8 & 0 & 0.704820 & -0.054652 & -0.180039 \\
\hline
\end{tabular}




$\begin{array}{rrrrrr}14 & 6 & 0 & 0.087959 & -1.251039 & 0.396379 \\ 15 & 6 & 0 & 0.194229 & -1.275674 & 1.922299 \\ 16 & 1 & 0 & -1.206233 & -3.462611 & -0.301402 \\ 17 & 1 & 0 & 0.392630 & 1.771522 & -0.932498 \\ 18 & 1 & 0 & 1.582781 & 1.968175 & 1.259305 \\ 19 & 1 & 0 & 0.080144 & 1.563842 & 2.112688 \\ 20 & 1 & 0 & 0.174130 & 3.034198 & 1.131783 \\ 21 & 1 & 0 & -3.137230 & 2.372189 & -0.753692 \\ 22 & 1 & 0 & -1.556379 & 3.141572 & -0.841840 \\ 23 & 1 & 0 & -2.315635 & 2.878193 & 0.724051 \\ 24 & 1 & 0 & -4.123671 & 0.697162 & -0.382418 \\ 25 & 1 & 0 & -3.821841 & -0.730483 & -1.373853 \\ 26 & 1 & 0 & -4.017207 & -0.893817 & 0.368724 \\ 27 & 1 & 0 & 0.698333 & -2.049072 & -0.024488 \\ 28 & 1 & 0 & 1.216223 & -1.044825 & 2.229898 \\ 29 & 1 & 0 & -0.063438 & -2.278610 & 2.274528 \\ 30 & 1 & 0 & -0.497113 & -0.571161 & 2.390915 \\ 31 & 1 & 0 & -2.853041 & -2.733831 & -0.691518 \\ --------------------------------------------------------\end{array}$

$\mathbf{c P}_{\mathrm{DX}}$

$\mathrm{E}(\mathrm{RB}+\mathrm{HF}-\mathrm{LYP})=-790.485989469 \quad \mathrm{~A} . \mathrm{U}$.

Zero-point correction $=0.254027$ (Hartree/Particle)

\begin{tabular}{|c|c|c|c|c|c|}
\hline \multirow{2}{*}{$\begin{array}{l}\text { Center } \\
\text { Number }\end{array}$} & \multirow{2}{*}{$\begin{array}{l}\text { Atomic } \\
\text { Number }\end{array}$} & \multirow{2}{*}{$\begin{array}{c}\text { Atomic } \\
\text { Type }\end{array}$} & \multicolumn{3}{|c|}{ Coordinates (Angstroms) } \\
\hline & & & $\mathrm{X}$ & $\mathrm{Y}$ & $\mathrm{Z}$ \\
\hline 1 & 9 & 0 & -1.633360 & 0.693654 & -1.552967 \\
\hline 2 & 5 & 0 & -1.962975 & -0.389657 & -0.805698 \\
\hline 3 & 9 & 0 & -1.744892 & -1.597274 & -1.391573 \\
\hline 4 & 9 & 0 & -3.112846 & -0.289271 & -0.095962 \\
\hline 5 & 6 & 0 & 1.817711 & 0.512069 & -0.225710 \\
\hline 6 & 6 & 0 & 0.784990 & 1.475725 & 0.217393 \\
\hline 7 & 6 & 0 & 1.615295 & -0.818222 & -0.076230 \\
\hline 8 & 6 & 0 & 0.860223 & 2.794650 & -0.016452 \\
\hline 9 & 6 & 0 & 0.310636 & -1.384684 & 0.445147 \\
\hline 10 & 6 & 0 & 0.410427 & -2.066956 & 1.814302 \\
\hline 11 & 6 & 0 & 2.617352 & -1.895548 & -0.421783 \\
\hline 12 & 6 & 0 & 3.074922 & 1.095372 & -0.827190 \\
\hline 13 & 8 & 0 & -0.750764 & -0.384680 & 0.473055 \\
\hline 14 & 6 & 0 & -0.322228 & 0.889989 & 1.068169 \\
\hline 15 & 6 & 0 & -1.540401 & 1.759526 & 1.334966 \\
\hline 16 & 1 & 0 & 0.096240 & 3.480262 & 0.328781 \\
\hline 17 & 1 & 0 & -0.055519 & -2.114349 & -0.277585 \\
\hline 18 & 1 & 0 & 0.775862 & -1.388647 & 2.590542 \\
\hline 19 & 1 & 0 & 1.106050 & -2.908663 & 1.756298 \\
\hline 20 & 1 & 0 & -0.569046 & -2.451106 & 2.109831 \\
\hline 21 & 1 & 0 & 3.338152 & -1.577670 & -1.175242 \\
\hline 22 & 1 & 0 & 2.110343 & -2.785425 & -0.811166 \\
\hline 23 & 1 & 0 & 3.185282 & -2.215143 & 0.461906 \\
\hline 24 & 1 & 0 & 3.815746 & 0.337752 & -1.078274 \\
\hline 25 & 1 & 0 & 3.545260 & 1.801438 & -0.133722 \\
\hline 26 & 1 & 0 & 2.845164 & 1.651176 & -1.743482 \\
\hline 27 & 1 & 0 & 0.109868 & 0.603732 & 2.034695 \\
\hline 28 & 1 & 0 & -1.249371 & 2.591404 & 1.981810 \\
\hline 29 & 1 & 0 & -2.305559 & 1.174304 & 1.846386 \\
\hline 30 & 1 & 0 & -1.961875 & 2.155112 & 0.410801 \\
\hline 31 & 1 & 0 & 1.680721 & 3.236045 & -0.568684 \\
\hline
\end{tabular}

